\title{
Annual Meeting Abstracts
}

Published online: 26 May 2012

C American College of Medical Toxicology 2012

The following are the abstracts from the scientific presentations of the 10th Annual Congress of the Asia-Pacific Association of Medical Toxicology (APAMT), which was held in Penang, Malaysia, in November 2011. Clinicians and researchers from over 20 countries attended this meeting, where more than 110 abstracts were showcased as either oral platform or poster presentations.

Nine young investigators from across the Asia-Pacific region were given scholarships to attend the meeting and to present their research as oral presentations. Their talks were mixed in with invited presentations on subjects relevant to Asia, including Chinese and Ayurvedic medicine toxicity, snake systematics and venom, pharmacovigilance, two plenary talks by recipients of APAMT Honorary Fellowships, and high-quality clinical and public health research. Two very successful symposia were held on fomepizole use and on recreational drug use in Asia-Pacific (hosted by American College of Medical Toxicology (ACMT)). The pre-congress focused on medical management of pesticide and recreational drug poisoning.

The next APAMT congress will be held in Hong Kong at the end of November 2012. More information on this congress and on previous congresses can be found at http://www.asiatox.org. We encourage members of ACMT to come to this meeting to hear about the fascinating toxicology characteristic of this region.

\section{Oral Presentations}

\section{(1) Agricultural Risk Index for Chronic Kidney Disease of Unknown Origin}

Jayasekara $\mathrm{JMKB}^{1}$, Dissanayake $\mathrm{DM}^{1}$, Gunaratna $\mathrm{MDN}^{2}$, Ranasinghe $\mathrm{P}^{2}$, Thilakarathna $\mathrm{S}^{3}$.

${ }^{1}$ Department of Pathology, Faculty of Medicine, University of Peradeniya, Peradeniya, Sri Lanka.
${ }^{2}$ Department of Mathematics, Faculty of Engineering, University of Moratuwa, Moratuwa, Sri Lanka.

${ }^{3}$ Department of Medical Laboratory Sciences, Faculty of Allied Health Sciences, University of Peradeniya, Peradeniya, Sri Lanka

Introduction: Chronic kidney disease due to unknown aetiology (CKD-U) is one of the growing health problems in Sri Lanka. About 8,000 diagnosed CKD-U patients are enduring treatment in the country largely in the North Central Region (NCR). Ninety percent $(90 \%)$ of the patients are farmers.

Objectives: The study is designed to calculate an agricultural risk index for an individual who lives in high risk areas of the country.

Methods and Materials: The study is a case-control study and $315 \mathrm{CKD}-\mathrm{U}$ patients and 321 normal healthy individuals were randomly selected from NCR. Age, sex and agriculture-related risk factors from both patients and controls were collected. Multiple linear regression logistic models were applied to calculate the risk index in terms of probability.

Results: The gender of the individual (male or female), age category ( $<45,45-60$ or $<60$ years), cultivating area ( $>1$ or $<1 \mathrm{ha}$ ), exchange of labour (high or low) and protective measures against agrochemicals (low or high) were considered for index calculation. According to the multiple linear regressions logistic models the maximum and minimum risks were calculated as 1.0 and 0.0 , respectively. The male individual whose age is greater than 60 years, low exchange of labour and low protective measures against agrochemicals had the highest risk of getting CKD-U the disease disregarding cultivating area (probability $=0.87$ ). Females with age less than 45 years with high labour exchange and high protective measures had the lowest risk (probability= $0.14)$ disregarding cultivating area.

Conclusion: The calculated risk index will be useful to identify the individuals at risk of developing CKD-U and to implement the preventive strategies for the disease in the 
high prevalent area. The individuals with age greater than 60 with sloppy agricultural activities had the maximum possibility of getting CKD-U probably due to long-term exposure to the aetiological agents and risk factors in this area.

\section{(2) Effect of Concentrated Water from Reservoirs of High-Prevalence Area for CKD of Unknown Origin in Sri Lanka on Mice}

JMKB Jayasekera ${ }^{1}$, DM Dissananyake ${ }^{1}$, Palitha Ratnayake ${ }^{2}$, W Wickramasinghe ${ }^{3}$, YA Radella ${ }^{4}$.

${ }^{1}$ Department of Pathology, Faculty of Medicine, University of Peradeniya, Peradeniya, Sri Lanka.

${ }^{2}$ Srimavo Bandaranayake Specialized Children Hospital, Peradeniya, Sri Lanka.

${ }^{3}$ National Environmental Toxicology Laboratories, University of Queensland, Brisbane, Queensland, Australia.

${ }^{4}$ Department of Medical Laboratory Science, Faculty of Allied Health Sciences, University of Peradeniya, Peradeniya, Sri Lanka.

Introduction: There is threateningly high prevalence of chronic kidney disease due to tubulointerstitial disease ending as chronic renal failure in the North Central Region of Sri Lanka. The epidemiology of the disease shows distribution of these patients around the water reservoirs and most of them are farmers.

Objectives: The aim of the study is to find the potential effects of concentrated water of the reservoirs in the high prevalent area by mouse bioassay.

Methods: Water of Padaviya Reservoir, supplying water to a high-disease-prevalent area, was concentrated 15 times by evaporation, being exposed to sunlight. The test group of mice (20) and control group (15) were fed with concentrated reservoir water and water from non-prevalence area, respectively. Water samples were analyzed for fluoride, $\mathrm{Na}+, \mathrm{K}+$ (ISE), heavy metals (ICP/mass spectrometry (MS)) and for cyanobacterial toxins microcystin (liquid chromatography (LC)/PDA) and cylindrospermopsin (LC/MS/MS).

Results: Interstitial nephritis was detected in $45 \%$ of test mice and only $6.5 \%$ of control group $(p<0.001)$. The analysis of concentrated water samples from Padaviya Reservoir showed significantly high content of fluoride $(2.25 \mathrm{mg} / \mathrm{L})$, Sodium $(225 \mathrm{mg} / \mathrm{L} ; p<0.05)$ than control samples. However, no increased levels of heavy metals were detected. The analysis showed presence of deoxycylindrospermopsin $(1.28 \mu \mathrm{g} / \mathrm{L}-\mathrm{DCYN})$ as the predominant isomer present over cylindrospermopsin which is unusual.

Conclusion: The results show the ability of the water of this reservoir to induce interstitial nephritis that could be due to the high salinity, fluoride, or DCYN. Although present in low levels, the possibility DCYN to induce interstitial nephritis needs to be investigated further as the epidemiological evidence is in favor of a cyanobacterial toxin. The long-term effects and safe levels for DCYN in drinking water and the effect of salinity and high fluoride content of water needs to be studied.

\section{(3) Referral Hospitals Provide Valid Toxicovigilance Signals for Acute Poisoning in Rural Sri Lanka}

Lalith Senarathna ${ }^{1,2}$, Nicholas A Buckley ${ }^{1,4}$, Shaluka $\mathrm{F}$ Jayamanna $^{1,3}$, Patrick Kelly ${ }^{2}$, Michael J Dibley ${ }^{2}$, Andrew H Dawson $^{1,4,5}$.

${ }^{1}$ South Asian Clinical Toxicology Research Collaboration, Faculty of Medicine, University of Peradeniya, Peradeniya, Sri Lanka.

${ }^{2}$ Sydney School of Public Health, University of Sydney, Sydney, NSW, Australia.

${ }^{3}$ Department of Clinical Medicine, Faculty of Medicine, University of Kelaniya, Kelaniya, Sri Lanka.

${ }^{4}$ Professorial Medicine Unit, POW Clinical School, University of New South Wales, Sydney, NSW, Australia.

${ }^{5}$ Royal Prince Alfred Clinical School, University of Sydney, Sidney, NSW, Australia

Objective: Rural areas of developing countries bear the burden of acute self-poisoning. Most patients receive treatment from peripheral hospitals before being transferred to referral hospitals. The epidemiology of acute poisoning is important but accurate data collection in such health systems can be difficult. We determined which easily collected dataset would be most representative of the true acute poisoning epidemiology.

Methods: We prospectively collected data from all acute poisoned patients admitted to all hospitals in Anuradhapura district in Sri Lanka from September 2008 to January 2010. Patients transferred from peripheral to referral hospitals were identified and linked. The 'observed' estimates of poisoning that would be found in data collected from primary hospitals, secondary referral hospitals and 'all admissions' (i.e. double-counting transfers) were compared with the most accurate data.

Findings: There were 3,813 poisoned patients in the district, of these, 3,111 first presented to a peripheral hospital and then 2,287 (73.5 \%) were transferred to referral hospital. Seven hundred two patients directly presented to the referral hospitals. There was no substantial difference in the age/ gender profile in any dataset. Secondary referral hospital data provided the closest estimate to the true epidemiology for the pattern of poisoning, total fatalities and case fatality (when compared to primary hospital data and "all admissions" data that double-counted transfers).

Conclusions: Epidemiology data of poisoning derived from secondary referral hospitals provided an estimate that was close to those found with far more labour-intensive methods 
using data linkage to track-transferred patients. Such models have potential in other developing world settings.

(4) Evaluation of Antidotal Effect of Intravenous Lipid Emulsion in Diazinon-Induced Acute Toxicity

Mohammad Moshiri ${ }^{1}$, Hossein Hosseinzadeh ${ }^{1}$, Maryam Vahabzadeh $^{1}$, Leila Etemad ${ }^{1}$.

${ }^{I}$ Department of Pharmacodynamics and Toxicology, School of Pharmacy, Mashhad University of Medical Science, Mashhad, Iran

Introduction: Diazinon (DZN) is a synthetic organophosphorus (OP) insecticide widely used in agricultural and household applications. OPs, particularly DZN, are highly lipid-soluble liquids. Intravenous lipid emulsion (ILE) has been shown to reduce toxicity caused by some lipid-soluble agents. We evaluated the antidote effect of ILE on acute toxicity of DZN.

Methods: Twenty-four non-pregnant Sprague-Dawley female rats weighting 200-250 g were divided into four groups consisting of six rats. All of the animals were treated orally with dose of $480 \mathrm{mg} / \mathrm{kg}(2 \times \mathrm{LD} 50)$ of DZN which was dissolved in corn oil and gavaged at the volume of $0.5 \mathrm{ml} / \mathrm{kg}$. Thirty minutes after administration of DZN, two groups were treated by either ILE $10 \%$ (ILE10) or NS (16 ml/ $\mathrm{kg}$ ) (NS16) that were infused for the duration of $15 \mathrm{~min}$. Another two groups were also treated by either ILE $20 \%$ (ILE20) or NS $(10 \mathrm{ml} / \mathrm{kg})(\mathrm{NS} 10)$ as above. The changes in body weight, diarrhea score, muscular power, fasciculation, convulsions and mortality rate of the animals were all monitored immediately after infusions and then every $6 \mathrm{~h}$ up to $48 \mathrm{~h}$.

Results: There was no significant difference in animals mean weight between different groups, during the observation period. In addition, during the 48-h observation, we could not find any difference in muscular power and diarrhea score between groups of ILE20-NS10 and ILE10-NS16 in comparison with each other, and neither ILE $10 \%$ nor ILE \%20 could not reduce mortality rate of animals or increase the survival time of rats.

Discussion: In conclusion, ILE seems to be unable to reverse DZN acute toxicity, because the major part of DZN converts into a 1,000 times more potent and 10 times less lipid-soluble product, diazoxon, prior to its access into the blood circulation

(5) Comet Assay Application for Evaluation of DNA Damage Induced by Sulphur Mustard in Khorassan Razavi Veterans

Seyed Adel Moallem, Mahdi Balali Mood, Effat Behravan, Shahryar Khateri.

Mashhad University of Medical Sciences, Janbazan Medical and Engineering Research Center, Tehran, Iran
Objective: Sulphur mustard (SM), also known as mustard gas, has been the most widely used chemical weapon. Over 100,000 Iranians were exposed to sulphur mustard during the Iran-Iraq war. SM causes DNA adducts and crosslinks and its genotoxicities have been evaluated in vitro. In this study, we have investigated the oxidative stress induced by SM in the veterans of Khorassan Razavi province by Comet assay, which is a sensitive and reliable method for assessing DNA damage. Alkaline conditions optimize the migration of single-stranded DNA, thus permitting an evaluation of single-stranded DNA breaks.

Methods: Blood samples were obtained from the veterans who had $>25 \%$ disability due to complications of SM poisoning and from their first-degree relatives as negative controls. Lymphocytes were isolated from blood samples using density gradient solution (Lymphopreb) and incubated in a dark $4^{\circ} \mathrm{C}$ fridge for $24 \mathrm{~h} . \mathrm{H}_{2} \mathrm{O}_{2}(25 \mu \mathrm{M})$ was used as the positive control. Microscopic slides were prepared and after incubation in cold lysing solution, alkaline electrophoresis was assessed. DNA break was measured based on percentage DNA tailing and tail moments and compared with the controls.

Results: SM induced significant DNA single-strand break compared with the negative controls. The genotoxic activity of $\mathrm{SM}$ was lower than $\mathrm{H}_{2} \mathrm{O}_{2}(25 \mu \mathrm{M} ; 36.5 \%$ tail DNA). This is presumably due to SM-induced cross-linking.

Conclusion: Cross-linking in DNA of SM-exposed lymphocytes interferes with DNA migration that can be detected as DNA strand breaks in the Comet assay. The percentage of DNA tailing remained significantly higher than unexposed individuals and confirms the DNA damage induced by SM.

(6) Persistent Organochlorine Pesticides in Farmers' Urine and Blood Samples in Pinang Tunggal Agricultural Areas, Penang-Malaysia

Razak Hj. Lajis, Che Nin Man, Juliana Mohd Noor

National Poison Centre, Universiti Sains Malaysia, Penang, Malaysia

Introduction: Since 1998, Malaysia has banned or deregistered many persistent organochlorine pesticides. Pesticides such as endrin, heptachlor, hexachlorobenzene, mirex, toxaphene and chlordecone (kepone) have never been registered for use in the country. However, some studies showed the presence of remnants of these pesticides in the environmental and human biological samples. Objective: The objective of this study is to determine the level of organochlorine pesticides (OCPs) in farmers' biological samples particularly in hair and urine samples by using gas chromatography-mass spectrometry (GC-MS). Methodology: OCPs selected in this study were HCHs $(\alpha-$, $\beta-, \gamma-$ and $\delta-H C H)$, DDTs (p,p'-DDT, p,p'-DDD and p, $p^{\prime}-$ 
DDE), aldrin, dieldrin, endosulfan sulphate, heptachlor, heptachlor epoxide and methoxychlor. This was a cross-sectional study that involved farmers working in agricultural areas and using pesticides during the time of the study. Hair and urine samples were collected from farmers who gave their consent to participate in the study. Method development and validation of the respective pesticides using GC-MS were established, and levels of the pesticides determined.

Results: Eight (8) and 10 out of 12 OCPs under study were detected in the hair and urine samples respectively. In the hair samples, the highest mean concentration of pesticide detected was methoxychlor $(3,389 \mathrm{ng} / \mathrm{g})$ whilst $\mathrm{p}, \mathrm{p}^{\prime}$-DDE was the lowest mean detected at $463 \mathrm{ng} / \mathrm{g}$. OCPs namely $\gamma$ hch, heptachlor, aldrin and dieldrin were not detected. In urine, the highest mean concentration of pesticide detected was dieldrin $(965 \mathrm{ng} / \mathrm{g})$ whilst $\mathrm{p}, \mathrm{p}^{\prime}$-DDE was the lowest at $221 \mathrm{ng} / \mathrm{g}$. $\beta$-hch and aldrin were not detected. On weigh to weigh basis, higher concentration of OCPs was detected in hair as compared to those found in urine.

Conclusion: This study has developed an assay for simultaneous quantification of selected OCPs which is simple, cost-effective and non-laborious. After so many years, traces of persistent OCPs could still be detected in human biological samples showing that the threat to human health still exists and is real.

\section{(7) New Role of Poison Center-the Quitline Service}

Sulastri S., Haslina H., Rahmat A., Razak L.J., Noor Azimah Z. National Poison Center, Universiti Sains Malaysia, Penang, Malaysia

Introduction: Smoking can be defined as chronic poisoning from tobacco use among smokers. However, findings from recent study showed that more and more smokers have intentions to quit. Apart from the support from quit clinics, evidence shows that telephone-based smoking cessation service or quitline has been found to be effective in helping smokers quit. In Malaysia, until 2005, no other agency or organization has been known to offer quitline service. This prompted the National Poison Centre of Malaysia to take on a new role in providing the quitline service.

Objective: The purpose of this study is to share the experience on quitline service provided within a national poison center setting.

Methods: Quitline incorporates an innovative easy-to-use interview system known as the Smoke-Free Online System. A registered client will undergo a preparatory session a week before the quit date. The client will then receive four follow-up sessions that provides motivational support to sustain quitting. Since 1 January 2011, the quitline service has been piloted in Malacca in conjunction with the Malacca Smoke-Free Project. Under this project, all patients who visit primary health care facilities in Malacca are screened for their smoking status. Smokers are then given brief advice and offered assistance to quit smoking. Result: Twenty-six health clinics and three hospitals in Melaka have joined the program. Until June end 2011, a total of 152 clients have registered and $95(62.5 \%)$ are actively in the follow-up sessions. Five (5.26\%) clients successfully quit on the targeted date and maintained their abstinence after followup at 1 month. The remaining clients are still in contact to set new quit dates. Most of the clients prefer to be contacted afteroffice hours or during their lunch break.

Conclusion: The quitline service has potential for expansion as it is well received by the general public. It offers a convenient alternative for smokers who are unable to attend quit smoking sessions at quit clinics. Poison centers with limited funding could also adopt this new role as the service offered incurs minimal additional operational cost and infrastructure.

\section{(8) Cases of Accidental Hydrocarbon Ingestion Referred to the Malaysian National Poison Center}

Sulastri S., Nur Afni A., Saed H.Z., Abdullah A.A., Rahmat A. National Poison Center of Malaysia, Universiti Sains Malaysia, Penang, Malaysia

Objective: Hydrocarbons-based products are among the most common chemicals used in everyday life, thus a potential poisoning risk. Although most hydrocarbon products may not cause appreciable systemic effects but aspiration of hydrocarbons into the lungs can cause chemical pneumonitis which could result in moderate to severe lung injury. The aim of the study is to describe the pattern of accidental hydrocarbon poisoning reported to the National Poison Center (NPC) in Malaysia over a 4-year period.

Methods: All poisoning cases involving hydrocarbon-based products referred to NPC between 1 January 2006 and 31 December 2009 were evaluated retrospectively. Reviewed data took into account the following factors: incidence, time of exposure, age category, route of uptake, and location of incident. SPSS version 15 was used for descriptive analysis of the data collected.

Results: Of the total 2,237 poisoning cases involving household products, 496 (22.2\%) cases were attributed to hydrocarbon products with $69 \%$ unintentional and $30.6 \%$ intentional cases been referred. There was an upward trends in accidental cases referred to the center from year 2006 with $75(15.1 \%)$ to 190 calls $(38.3 \%)$ in 2009 . More than half of these cases involved children. The highest incidence was poisoning involving kerosene (40.9\%) followed by thinner (32.2\%), petrol (23.1\%) and others hydrocarbon-based product (3.8\%). Majority of these poisonings happened at home (93.9\%), and ingestion (98\%) was the commonest route of exposure. 
Conclusion: Kerosene being the most common agent involve in hydrocarbon poisoning could be due to its clear properties which can be easily mistaken for water. The finding highlights the need to emphasize on proper labeling and storage of chemicals especially in home and its surrounding. Higher prevalence of poisoning among children also warrants a comprehensive action including educating parents on dangerous substances in home surroundings and the importance of proper storage to curb accidental poisoning.

(9) Toxic Elements of Mercury, Lead, Chromium, Arsenic, Aluminium and Cadmium in Drinking and Agricultural Water Wells of Mashhad, Iran

Hadis Yousefzadeh $^{1,2}$, Reza Mousavi ${ }^{1,2}$, Mahmood Sadeghi ${ }^{1,2}$, Mohssen Namaei Ghassemi ${ }^{1,2}$, Kazem Eshaghian ${ }^{1,2}$, Vali Allah Moradi $^{1,2}$, Mehr-Angiz Mokhtari ${ }^{1,2}$, Gholamhassan Danay ${ }^{1,2}$, Maryam Fakhary ${ }^{1,2}$, Mahdi Balali-Mood ${ }^{1,2}$.

${ }^{I}$ Young Researchers Club, Islamic Azad University, Mashhad Branch, Mashhad, Iran.

${ }^{2}$ Medical Toxicology Research Center, Faculty of Medicine, Mashhad University of Medical Sciences Regional Water Organization, Khayyam Boulevard, Khayyam 35, 9185916196, Mashhad, Iran.

Contamination of drinking and agricultural waters by chemicals particularly toxic elements is a cause of concern for the environmental and health authorities. Increased population, shortage of water in summer and industrialization resulted in digging many water wells in Mashhad. It was thus aimed to determine concentrations of mercury, lead, chromium, arsenic, aluminium and cadmium in waters of 10 drinking and 4 agriculture tube water wells of Mashhad and compare them with the international standards. Sampling was performed three times in March, May and July 2010 after a pilot study, which had been done in April 2009. Toxic elements concentrations were determined by different techniques of an atomic absorption spectrophotometry in the laboratory of medical toxicology research centre. $\mathrm{Hg}$ and $\mathrm{Pb}$ concentrations of the drinking water samples exceeded the WHO standards of 1 and $10 \mu \mathrm{g} / \mathrm{L}$ in 70 and $30 \%$ of the samples, respectively. Geochemistry map of the area revealed that the lead contamination was related to anthropogenic activities rather than geologic reasons. Maximum concentrations of $\mathrm{Pb}$ and $\mathrm{Cr}$ were the same $(90 \mu \mathrm{g} / \mathrm{L})$ despite a poor correlation $(r=-0.13)$ between them. Mercury contamination of agricultural water also exceeded the FAO standard $(1 \mu \mathrm{g} / \mathrm{L})$ in $50 \%$ of the studied sites with a maximum $7.5 \mu \mathrm{g} / \mathrm{L}$. Both waters were contaminated mostly by mercury, followed by lead, chromium, arsenic, aluminium and cadmium. Further investigations on toxic chemicals, particularly the toxic elements in the drinking and agricultural waters at regular intervals in different sites of Mashhad waters including the wells are recommended.
(10) A Simple Modified Bicarbonate Regimen for Urine Alkalinization in Moderate Pediatric Salicylate Poisoning in the Emergency Department

Ong Yong-Kwang Gene.

KK Women's and Children's Hospital, Little India, Singapore

A 4-year-old Indian girl was seen in our emergency department for unintentional ingestion of topical medication oil with subsequent salicylate poisoning. Serum levels were $52 \mathrm{mg} / \mathrm{dL}$ at $12 \mathrm{~h}$ after ingestion. She was started on urine alkalization therapy to enhance salicylate elimination. This was achieved by a bicarbonate bolus of $1 \mathrm{mEq} / \mathrm{kg}$ for an hour and a continuous bicarbonate-potassium-dextrose combination infusion. The infusion regimen was modified from adult recommendations to tailor for pediatric physiological requirements in a young child. This consisted of a combination solution of dextrose $5 \%$-sodium bicarbonate-potassium chloride with similar sodium content as half-strength $(0.45 \%)$ saline and supplemental potassium, which is crucial for effective urine alkalinization. The combination fluid was administered at a rate 1.5 times her maintenance fluid requirement to achieve a urine output of 1.5 to $2 \mathrm{~mL} / \mathrm{kg}$ per hour and a urine $\mathrm{pH}$ of 7.5 to 8.5 . This regimen was well tolerated with good outcome. Many pediatricians and toxicologists achieve urine alkalinization by giving multiple bicarbonate boluses and have separate hydration fluids with dextrose and supplemental potassium. These regimens may involve complex calculations and multiple infusions that may lead to increased risk of calculation and medication errors especially in the busy emergency department setting. This case report highlights the use of a simple modified urine alkalinization regimen for moderate salicylate poisoning in a young child in the emergency department.

\section{(11) Chronic Kidney Disease Following Snake Bite Envenoming in Sri Lanka}

H.M.N.J. Herath ${ }^{1}$, A.W.M Wazil ${ }^{1}$, D.T.D.J Abeysekara ${ }^{1}$, K.G.A.D Weerakoon ${ }^{2}$, N.D.C Jeewani1, N.V.I Rathnathunga ${ }^{3}$, E.H.C.K Bandara ${ }^{1}$, S.A.M Kularatne ${ }^{4}$.

${ }^{1}$ Nephrology and Transplant Unit, Teaching Hospital, Kandy, Sri Lanka.

${ }^{2}$ Departmant of Parasitology, Faculty of Medicine and Allied Sciences, Rajarata University of Sri Lanka, Mihintale, Sri Lanka.

${ }^{3}$ Department of Pathology, Faculty of Medicine, University of Peradeniya, Peradeniya, Sri Lanka.

${ }^{4}$ Department of Medicine, Faculty of Medicine, University of Peradeniya, Peradeniya, Sri Lanka.

Background: The evidence to support the development of chronic kidney disease (CKD) following snake bites was generated from a retrospective study, renal histology and 
comparative analysis with an another aetiology (leptospirosis) in the Nephrology Unit, Kandy, Sri Lanka.

Methods: A series of 54 patients who had acute kidney injury (AKI) after snake bite was assessed for CKD, compared with a series of 44 leptospirosis cases with AKI. The primary outcome measure was the failure of renal functions to return to normal in 1 year.

Results: The mean age of the group was 50 years (standard deviation (SD), 13 years) and 39 (72\%) were men. The offending snakes were Russell's viper and hump-nosed viper in $15(28 \%)$ and $13(24 \%)$ patients, respectively. At 1 year, 20 patients (37\%) developed CKD (CKD group) and the rest $(63 \%)$ had recovered (recovered group). The acute stage serum creatinine was high in both groups with no difference (on admission, $p=0.134$; on discharge, $p=$ 0.323 ), but CKD group showed significantly high serum creatinine at 2 months after AKI $(p=0.004)$. Mean duration of renal replacement therapy of the Recovered group and CKD group were $7(\mathrm{SD}, 5)$ and 16 (SD, 12) days respectively $(p=0.015)$. Compared to snake bite group, three patients $(7 \%)$ in control group (leptospirosis) developed $\mathrm{CKD}$, the difference is significant $(p=0.001)$. Renal histology of six CKD snake bite patients showed predominant glomerular sclerosis and interstitial nephritis.

Conclusions: Chronic kidney disease is a potential complication secondary to AKI following snake envenoming.

\section{(12) Risk Factors Associated with Two Viperid Snake} Bites in Sri Lanka: a Case-Control Study

K.G.A.D Weerakoon ${ }^{1}$, S.A.M Kularatne ${ }^{2}$, A. Rathnathilake ${ }^{3}$, P.V.R Kumarasiri ${ }^{4}$.

${ }^{1}$ Departmant of Parasitology, Faculty of Medicine and Allied Sciences, Rajarata University of Sri Lanka, Mihintale, Sri Lanka.

${ }^{2}$ Department of Medicine, Faculty of Medicine, University of Peradeniya, Peradeniya, Sri Lanka.

${ }^{3}$ General Hospital, Kurunegala, Sri Lanka.

${ }^{4}$ Department of Community Medicine, Faculty of Medicine, University of Peradeniya, Peradeniya, Sri Lanka.

In Sri Lanka, two viperid snakes Russell's viper (Daboia russelii) and hump-nosed viper (Hypnale species) are responsible for most of the venomous snake bites. These two snakes share somewhat similar habitats and behavior. The aim of this study was to assess the environmental and the behavioral risk factors of victims that promoted the bites due to these two snakes. The cases were recruited from consecutive admissions to the General Hospital, Kurunegala with proven viperid bites. Age- and gender-matched controls were selected from relatives and neighborhoods of cases in a ratio of 1 case $/ 2$ controls. There were 56 cases and 112 controls with mean age 44 years (standard deviation (SD)
15 ) and 45 years (SD 22), respectively. Of the 14 risk factors assessed, 8 risk factors showed a significant association with viperid bites. Among these, the risk factors at the time of bite were: (1) not using protective foot wear ( $p=0.03$; odds ratio (OR), $95 \%$ confidence interval $(\mathrm{CI})=0.14(0.02$ $1.09))$, (2) being in an ill-lit place $(p<0.001$, OR, $95 \%$ $\mathrm{CI}=6.6(3.25-13.4))$, (3) being in outdoor places $(p<0.001$, OR, $95 \% \mathrm{CI}=148.8(43.7-506))$, (4) working in a field ( $p<$ 0.001, OR, $95 \% \mathrm{CI}=175$ (31.4-976)). The general risk factors that reached significant association were: (1) occupation as a field worker $(p<0.001$, OR, $95 \% \mathrm{CI}=5.3(2.3-$ $12)$ ), (2) low level of financial status $(p<0.001, \mathrm{OR}, 95 \%$ $\mathrm{CI}=9.9(2.75-35.5))$, (3)lack of attached toilet $(p<0.001$, OR, $95 \% \mathrm{CI}=7.38(2.15-25.3))$, (4) presence of rats in the compounds $(p<0.001$, OR, $95 \% \mathrm{CI}=11(4.88-24.9))$. Identifying easily remediable risk factors would help in preventing viperid snake bites.

\section{(13) Short-Term Effects of Cyanobacterial Toxin Extracts on Mice}

D. M. Dissananyake ${ }^{1}$, JMKB Jayasekera ${ }^{1}$, Palitha Ratnayake ${ }^{2}$, W. Wickramasinghe ${ }^{3}$, Y. A. Radella ${ }^{4}$.

${ }^{1}$ Department of Pathology, Faculty of Medicine, University of Peradeniya, Peradeniya, Sri Lanka.

${ }^{2}$ Srimavo Bandaranayake Specialized Children Hospital, Peradeniya, Sri Lanka.

${ }^{3}$ National Environmental Toxicology Laboratory, University of Queensland, Brisbane, Queensland, Australia.

${ }^{4}$ Department of Medical Laboratory Science, Faculty of Allied Health Sciences, University of Peradeniya, Peradeniya, Sri Lanka.

Introduction: The chronic kidney disease of unknown origin in Sri Lanka shows clustering of these patients around the water reservoirs and parallel variations of incidence over time in CKD-U and alcoholic liver disease in the North Central Region, indicating the possibility of a common aetiological agent.

Objectives: The aim of this study is to find the short-term effects of extracts of cyanobacteria isolated from the affected region on mice.

Methods: Diluted extracts of microcystis, cylindrospermopsis and lyngbia bloom was fed to a group of five, seven and ten mice, respectively, for a week. Another five mice fed with diluted extracts of microcystis bloom for 1 week of followed by 2 weeks of normal water. The control group of mice (10) was given normal water for a week. Cyanobacterial extracts were analyzed for microcystisn, deoxy-cylindrospermopsin $(\mathrm{DCYN})$ and cylindropsermopsin (CYN).

Results: Acute tubular necrosis (ATN) was detected in $5 / 5$ mice fed with extracts of microcystis bloom that contained microcystin $(65 \mu \mathrm{g} / \mathrm{l}), \mathrm{DCYN}(2.1 \mu \mathrm{g} / \mathrm{l})$ and CYN while $2 / 5$ 
mice had ATN when this extract was followed by 2 weeks of normal water. One out of seven mice fed with cylindrospermopsis bloom that contained DCYN $(29.5 \mu \mathrm{g} / \mathrm{l})$ and CYN $(0.7 \mu \mathrm{g} / \mathrm{l})$ had ATN. Six out of ten mice fed on Lyngbia bloom containing CYN $(1.7 \mu \mathrm{g} / \mathrm{l})$ and DCYN $(0.5 \mu \mathrm{g} / \mathrm{l}) \mathrm{had}$ acute tubular necrosis. All control mice had normal tubules. Conclusion: The results show the ability of the cyanobacterial extracts to induce ATN in mice in the given concentrations. The ability of the kidneys to recover is suggested by the less frequent abnormalities seen after normal water has been supplied 2 weeks post-microcystis poisoning. As DCYN was available in all three extracts causing ATN, the ability of DCYN on its own to induce tubular necrosis even at low concentrations need to be investigated.

\section{(14) Managing Indian Common Krait (Bungarus caeruleus)} Bites in Resource Poor Setting

Ashish Bhalla, Navneet Sharma, Surjit Singh.

Postgraduate Institute of Medical education and Research, Chandigarh, India

Introduction: Bungarus caeruleus (Indian common krait) bite during monsoons is common in Northwest India. Respiratory failure is responsible for high mortality in the victims. The limited availability of mechanical ventilators can hamper treatment. In this study, we are reporting our experience with manual ventilation using Ambu bag in patients with $B$. ceruleus bite.

Subjects and Methods: This prospective study was undertaken between June 2009 and December 2009. All consecutive patients with common krait bite receiving manual ventilation using Ambu bag were included. The patients receiving mechanical ventilation by ventilators within the first $12 \mathrm{~h}$ of arrival were excluded from the study. Polyvalent anti-snake venom was administered as per the national snake bite protocol. Manual ventilation was provided using Ambu bag and the patients friends/relatives took turns every $30 \mathrm{~min}$ to ventilate. The primary end point was improvement in neuroparalysis.

Results: Thirty-four patients were admitted during the study period. All the patients except two came from rural areas and were brought between June and September. Majority of the patients were bitten at night time while sleeping. The mean time interval between bite and arrival to hospital was $4.5 \mathrm{~h}$. Twenty-four patients developed respiratory involvement and 20 were manually ventilated using Ambu bag. Mean duration of manual ventilation was $34.6 \mathrm{~h}$. All the patients ventilated manually, except one, survived. There were no major complications observed.

Conclusion: Manual ventilation in respiratory failure following common krait bite is an effective alternative in resource constraint setting.
(15) A Novel Antivenom-Bungarus candidus and Bungarus multicinctus (BC-BM)-Was Produced in Vietnam and a Controlled Clinical Trial of This AV in Envenomated Patients by (BC-BM)

Kiem Xuan Trinh, Tuyen Danh Thai. Bach Mai Hospital, Hanoi, Vietnam

Introduction: At APAMT 2010, the President of IST stated: "It is estimated that there may be about 5 million snakebites per year in the World, out of which 125,000 victims die as a result, about half of this death occur in Asia." It is about 30,000 snakebites annually in Vietnam, Bungarus candidus (BC) in the South and Bungarus multicinctus (BM) in the North are the most important snakes in medicine with very high mortality $(>20 \%)$ because there was no $(\mathrm{BC}-\mathrm{BM})$ antivenom (AV) available in the world. Since 2000, (BC$\mathrm{BM}) \mathrm{AV}$ has been produced and met the standards of $\mathrm{Na}-$ tional Institute for Control of Vaccine and Biological Products, $\mathrm{MOH}$, Vietnam. A controlled clinical trial of the novel bivalent antivenom in patients envenomed by $\mathrm{BC}$ and $\mathrm{BM}$ has been studied in response to this situation.

Materials and Methods: $\mathrm{BC}$ and $\mathrm{BM}$ antigen was made from venom of 100 snakes (50 $\mathrm{BC}$ and $50 \mathrm{BM}$ ). Three horses were increasingly immunized by (BC-BM) antigen in every month for 1 year. Plasmapheresis was performed after seven injections. Pool horse plasma was purified to make $\mathrm{F}\left(\mathrm{ab}^{\prime}\right)_{2}$ AV. After passing national quality control, a controlled clinical trial of a new $\mathrm{AV}$ in 123 patients (BC, 42; BM, 81) envenomed by (BCBM) venom (group A) have been studied for 3 years (20042007). All patients, who fulfilled the inclusion criteria respectively 2 years before, were enrolled and treated with optimal supportive therapy in the ICU (group B) for comparison.

Results: Bivalent (BC-BM) AV F( $\left.a b^{\prime}\right)_{2}$ was produced and passed National Institute Control of Vaccine and Biological Products, $\mathrm{MOH}$, Vietnam with safety (sterility and nonpyrogen) and potency at $267.5 \mathrm{LD}_{50}$ /vial. Comparison between the two groups (A and B): group A had a shorter duration of muscle paralysis of limbs $(p<0.001)$, of diaphragm $(p<0.001)$, and of ptosis $(p<0.001)$. The duration of mechanical ventilation and length of ICU stay were shorter $(p<0.001)$. The rate of ventilator-associated pneumonia was lower $(p<0.02)$. Adverse reactions to AV were $7.4 \%$.

Conclusions: The safety and efficacy of the new AV clearly demonstrated in this controlled clinical trial of patients envenomed by $B$. candidus and $B$. multicinctus. Bivalent (BC-BM) AV was made successfully in Vietnam.

\section{(16) Tramadol-Induced Apnea}

Hossein Hassanian-Moghaddam, Hoorvash Farajidana. Department of Clinical Toxicology, Shahid Beheshti University of Medical Sciences, Tehran, Iran 
Background and Objectives: In contrast with other opioids, few cases of tramadol-related respiratory depression have been described. To determine the prevalence and predisposing factors for tramadol-related apnea in the patients who had referred after its overdose or use of its therapeutic dose, current study was done.

Materials and Methods: All patients referred to LoghmanHakim Poison Hospital (February 2009 to April 2010) due to pure tramadol intoxication/complication were included. The patients' data including age, gender, ingested dose by history, manner of poisoning, history of tramadol abuse, seizure, and apnea occurrence were recorded. Statistical analysis was done using SPSS software.

Results: A total of 525 patients were included in the study. Mean age was $22.8 \pm 6.9$ years, predominantly males $(70.1 \%)$ with deliberate self-poisoning $(359 ; 68.4 \%)$ or abuse (146; 27.8 ), and past history of tramadol abuse (204; $38.9 \%)$. Two hundred forty-two $(46.1 \%)$ patients experienced seizure one episode or more $(30 ; 5.8 \%)$. Twenty patients $(3.8 \%)$ experienced apnea and required respiratory support $(17 ; 85 \%)$ or naloxone administration $(3 ; 15 \%)$ all before $24 \mathrm{~h}$ postingestion ( $7.7 \pm 7 \mathrm{~h}$, range $1-24)$. The mean dose ingested by history was 2,125 $\pm 1,360 \mathrm{mg}$ ( $\mathrm{min} 200 \mathrm{mg}$, $\max 4,600 \mathrm{mg}$ ) among these patients compare to rest with $1,383 \pm 1,088 \mathrm{mg}$ (min $100 \mathrm{mg}, \max 6,000 \mathrm{mg} ; p<0.002$ ). History of tramadol abuse was positive in nine (45\%) of the apnea patients. Seizure episode had occurred once in five $(20 \%)$ while one episode happened after naloxone administration revealing less episodes of seizure in apnea patients. There was no positive history of previous medical illness in apnea patients. A 40year-old man died after respiratory arrest following oral abuse of 1,000 mg tramadol but without co-ingestion.

Conclusions: The present retrospective study shows that although seizure is common in tramadol toxicity, respiratory depression and apnea is a life-threatening condition with $3.8 \%$ prevalence which needs more attention. Depend on genetic backgrounds and metabolism rate of tramadol to its active metabolite this prevalence could be different in other ethnic populations.

\section{(17) Acetaminophen Psi Nomogram: a Sensitive} and Specific Clinical Tool for Predicting Hepatotoxicity Secondary to Acute Acetaminophen Overdose

Summon Chomchai ${ }^{1}$, Chulathida Chomchai ${ }^{2}$, Nongnuch Lawattanatrakul ${ }^{1}$.

${ }^{1}$ Faculty of Medicine Siriraj Hospital, Mahidol University, Bangkok, Thailand.

${ }^{2}$ Mahidol University International College, Mahidol University, Nakorn Pathom, Thailand

Background: Acetaminophen overdose is one of the most common overdoses in Thailand. After completion of $N$ - acetylcysteine (NAC) therapy, additional aminotransferase levels are routinely obtained in order to assess the need for further follow-up or NAC therapy. Using acetaminophen level and lag-time-before-NAC-therapy, a composite value, the acetaminophen psi parameter (APP) has been proven to predict hepatotoxicity. The acetaminophen psi nomogram (APN) is derived based on the APP. It estimates individual patients' risk by six hepatotoxic risk cutoff lines (HepRCL) at $1,2,5,10,20$ and $50 \%$. We are reporting the validation of the APN using existing patient data with known hepatotoxicity outcomes.

Materials and Methods: This is a 4.5-year, retrospective review of patients with acute acetaminophen overdose presenting to Siriraj Hospital in Bangkok Thailand and treated with NAC. Patients are classified with APN's HepRCLs. The hepatotoxicity, which is the outcome of interest, is defined as aminotransferase 1,000 IU/L or more. The validity of the APN is reported as sensitivity and specificity.

Results: One hundred and sixty one patients aged (mean (SD)) 22.95(6.53) years were enrolled. Twenty five patients (15.5\%) developed hepatotoxicity. The number and percent of total patients, below each of the HepRCLs 1, 2, 5, 10, 20 and $50 \%$ are as follows: 73 (45.3\%), 76 (47.2\%), 92 (57.1\%), 107 (66.5\%), 124 (77.9\%) and 144 (89.4\%). Their corresponding sensitivities are 100, 100, 100, 92.0, 72.0 and $40.0 \%$, respectively. The 20 and $50 \%$ HepRCLs have specificities of 86.0 and $94.9 \%$.

Discussion and Conclusion: Acetaminophen psi nomogram, derived from existing acetaminophen level and timeto-NAC therapy, enables clinicians to predict individual's hepatotoxicity risk with high sensitivity and specificity. Such prediction can alleviate the need for further laboratory monitoring in as much as $57 \%$ of patients. This allows for allocation of limited healthcare resources to be focused on those with greatest predicted risk.

\section{(18) Multiple Organ Failure After Oxalic Acid Ingestion}

Cherie Grace G. Quingking ${ }^{1,2}$, Lynn Crisanta Panganiban ${ }^{1,2}$, Joselito C. Pascual ${ }^{1,2}$.

${ }^{1}$ National Poison Management and Control Center, University of the Philippines, Philippine General Hospital, Manila, Philippines

${ }^{2}$ Department of Health-East Avenue Medical Center Poison Control Center, Quezon City, Philippines

Introduction: Oxalic acid ingestion, available as laundry bleach, is an emerging agent among self-poisoning cases referred to the poison center. Ingestion of oxalic acid was associated with high case fatality.

Case Presentation: This is a case of 28-year-old man who presented with systemic complications after intentional oxalic acid ingestion. Patient was seen in the Emergency 
Department with abdominal pain following ingestion of $10 \mathrm{~g}$ oxalic acid mixed with water. Caustic injury was initially ruled out with normal endoscopic findings. Acute kidney injury evidenced by acute oliguric renal failure ensued within 6 h. Patient underwent five sessions of hemodialysis and before renal function indices returned to normal and calcium oxalate in the urine was no longer evident. Other systemic manifestations noted were metabolic acidosis with elevated anion gap with persistent hypocalcemia and episodes of QT prolongation. Respiratory distress developed on the third day accompanied with signs of acute lung injury. Supportive management was given with hemodialysis to facilitate elimination of calcium oxalate. Supportive mechanical ventilation, hydration, and electrolyte correction were given. $\mathrm{N}$ Acetylcysteine as nebulization to address lung injury and potassium citrate postulated to inhibit calcium oxalate supersaturation in the renal tubules were included in the treatment regimen to address organ-specific injury from oxalate. Patient was discharged after intensive care.

Discussion: Pathophysiologic findings following oxalic acid ingestion results from intraluminar crystal deposition with renal biopsy specimen showing the degeneration of the renal tubular epithelial cells associated with intracellular calcium oxalate crystal deposition resulting to acute renal failure. Deposition of the formed oxalate crystals may also occur in other organ systems. This explains the multiorgan failure exhibited in the course of the patient. Lessons Learned: Early identification of systemic complications of a caustic substance is imperative with adequate supportive management to avoid morbidity. It is recommended that oxalic acid ingestion be included in the continued toxicovigilance, and promotion of regulation controls on availability of toxic household chemicals be implemented.

\section{(19) Adverse Drug Events in Hospitalized Patients with Acetaminophen Overdose Treated with Intravenous $\mathrm{N}$-Acetylcysteine}

Sa'ed H Zyoud ${ }^{1}$, Rahmat Awang ${ }^{1}$, Syed Azhar Syed Sulaiman ${ }^{2}$.

${ }^{1}$ WHO Collaborating Centre for Drug Information, Clinical Toxicology Program, National Poison Centre, Universiti Sains Malaysia (USM), 11800 Penang, Malaysia.

${ }^{2}$ Clinical Pharmacy Program, School of Pharmaceutical Sciences, Universiti Sains Malaysia (USM), 11800 Penang, Malaysia.

Objectives: Intravenous $N$-acetylcysteine (IV-NAC) is widely recognized as the antidote of choice for acetaminophen overdose. However, its use is not without adverse drug reactions (ADR) which might affect therapeutic outcome or lead to treatment delay. The aims of this study were to investigate the type and incidence of ADR induced by IV-
NAC in patients treated for acetaminophen overdose and to assess the causality of individual ADR to IV-NAC using Naranjo's algorithm.

Methods: This is a retrospective study of patients admitted to the hospital for acute acetaminophen overdose over a period of 5 years (January 1, 2004 to December 31, 2008). The primary outcome of interest in this study was the occurrence of ADR during NAC administration. The probability of an ADR was assessed using the Naranjo algorithm, which consists of ten questions, and has been used to determine the likelihood that an ADR was related to a specific medication. The Naranjo score takes into account other possible influences such as drugs or disease. The association scores were: $\geq 9=$ "definite", 5 to $8=$ "probable", 1 to $4=$ "possible", and $0=$ "doubtful".

Results: During the study period, 305 patients with a diagnosis of overdose of paracetamol-containing compounds were admitted to the hospital for monitoring and treatment. Different types of ADR occurred in 137 patients (137/305; $44.9 \%)$. Of those patients who had an ADR, 98 (98/137; $71.5 \%)$ had been treated with IV-NAC and $39(39 / 137$; $28.5 \%)$ had not $(p<0.001)$. Comparison of different ADR in all patients showed that the following ADR were significantly associated with IV-NAC administration: nausea ( $p=$ $0.004)$, vomiting $(p<0.001)$, flushing $(p<0.001)$, rash $(p<$ $0.001)$, pruritus $(p<0.001)$, chest pain $(p=0.001)$, bronchospasm $(p=0.015)$, coughing $(p=0.017)$, headache $(p<$ $0.001)$, dizziness $(p<0.001)$, convulsion $(p=0.035)$, and hypotension $(p=0.001)$. Based on Naranjo's algorithm, 226 events were judged to be NAC related- $31.1 \%$ probably and $67.9 \%$ possibly drug related. None of the events were definitely drug related.

Conclusions: Adverse drug reactions to IV-NAC were common among patients with acetaminophen overdose but mostly minor, and that all reported adverse reactions were easily managed.

\section{(20) Follow-up Health and Environmental Assessment of Communities Exposed to Bunker Oil in Guimaras Province 15 Months After the Spill}

Panganiban $\mathrm{LR}^{1}$, Rivera $\mathrm{ATFR}^{2}$, Antonio $\mathrm{VP}^{3}$, Diqouino $\mathrm{CC}^{1}$, Reyes JPR ${ }^{1}$.

${ }^{I}$ National Poison Management and Control Center, UP College of Medicine, Philippine General Hospital, Manila, Philippines.

${ }^{2}$ National Center for Disease Prevention and Control, Department of Health, Sta. Cruz, Manila, Philippines.

${ }^{3}$ East Avenue Medical Center Poison Control Center, Quezon City, Philippines

Introduction: In 2006, massive oil spill occurred in Guimaras province resulting to the release of chemicals into the environment, severely affecting 14 communities. Seven 
hundred eighty-seven residents were examined and clinical findings were consistent with acute exposure. Environmental samples showed elevated lead levels and polycyclic aromatic hydrocarbons (PAH). Because of the potential chronic effects of bunker oil exposure, a follow-up health assessment among the exposed residents was necessary.

Objectives: The objective of this study is to assess current health status and monitor environmental exposures of residents and identify acute and/or chronic health effects.

Methodology: This was a cross-sectional study of exposed residents who provided consent for follow-up health evaluation. Repeat environmental monitoring was also done. Results/Discussion: Two hundred twenty residents from five islands and 244 from seven mainland communities were examined in December 2007 and January 2008. More residents from island communities claimed continuous exposures and $68.2 \%$ were sick. Acute effects were dizziness, headache, nausea, chest pain, and difficulty of breathing, similar to 2006 complaints. There were more abnormal physical examination findings among island community residents. Lower hemoglobin, platelet count and reticulocyte count, methemoglobin, higher sulfhemoglobin were observed compared to 2006 findings. Peripheral blood smears showed anemia and relative eosinophilia. Blood lead levels were higher in mainland community residents. Lead levels in drinking water were more elevated in the mainland communities. Blood PAH and drinking water PAH were non-detectable. Bunker oil was evident in the communities during the hot season when hardened bunker oil would melt and evaporate into the immediate environment. Residents claimed that the oil is carried to the shore during high tide. This was more evident in the island communities where clean-up was not as extensive as in the mainland communities. The abnormal hematologic picture may be signs of chronic bunker oil exposure.

Conclusion: The residents are continuously exposed to bunker oil manifesting with acute and possible chronic effects.

\section{(21) Fatal Aluminium Phosphide Poisoning in Bangladesh: a Case Series}

Amin MR ${ }^{1}$, Basher $\mathrm{A}^{2}$, Chowdhury FR ${ }^{3}$, Faiz MA ${ }^{4}$.

${ }^{1}$ Dhaka Medical College, Dhaka, Bangladesh.

${ }^{2}$ Kashba Upazilla Health Complex, Brammonbaria, Bangladesh.

${ }^{3}$ MAG Osmani Medical College, Sylhet, Bangladesh.

${ }^{4}$ Sir Salimullah Medical College, Dhaka, Bangladesh

Introduction: Aluminium phosphide (ALP) is a solid fumigant that is used as a rodenticide for grain preservation and is widely available in rural areas. The commonest way of aluminium phosphide poisoning is by ingestion; death is mostly due to cardiac and/or metabolic toxicity by its active ingredient phosphine. Here is a case series of aluminium phosphide poisoning in Bangladesh that presented features of cardiotoxicity and circulatory failure.

Case Reports: From April 2008 to March 2009, six patients (three male, three female) were admitted to Dhaka Medical College Hospital after ingesting aluminium phosphide tablets with an intention of deliberate self-harm due to family disputes. Five patients of 14-30 years were admitted 3-12 h after ingestion of the tablets and had symptoms of vomiting, abdominal pain, restlessness and hypotension and they were treated conservatively but died 5-20 h after admission. A 30-year-old male was admitted $3 \mathrm{~h}$ after ingestion of two tablets of aluminium phosphide. On admission, his vital parameters were normal, but $10 \mathrm{~h}$ later, he developed restlessness, irritability, drowsiness and circulatory failure with a respiratory rate of 24 per minute, a Glasgow coma score of 5 , and dilated pupils reacting to light. Subsequent exams revealed severe metabolic acidosis, anterior septal ischaemia, anterior wall hypokinesia with moderate left ventricular dysfunction, normal serum electrolytes, serum creatinine $3.2 \mathrm{mg} / \mathrm{dl}$. In spite of high-dose ionotropic and intensive care support and treated by magnesium sulphate and sodium bicarbonate, he developed progressive metabolic acidosis, renal failure and died from cardiac arrest 3 days after ingestion of the poison.

Discussion: Recently, poisoning due to rodenticide (ALP) is increasing because of its easy availability. This case series suggests that special management guidelines and training for physicians and other healthcare workers should be considered; and additional research is needed to determine the best therapy and improve survival.

\section{(22) Measuring Lead Levels in Common Children-Exposed} Environment, Mashhad, Iran, 2011

Alizadeh A, Alizadeh J, Afshari R, Moradi V, Mokhtari M, Zamanipour M.

Medical Toxicology Centre, Imam Reza (p) Hospital, Mashhad University of Medical Sciences Mashhad, Iran

Background: Lead is one of the nonessential heavy metals. It could induce disturbances in nervous system, hematopoietic and renal function in human. Children are more susceptible to hazards of lead than adults as neural network development happens in childhood. In addition, the absorption rate of lead through the gastrointestinal tract in infants and children is higher. They also present greater tendency for exposure to their contaminated hands with leaded materials.

Objective: The purpose of this study is to have a measurement of lead levels in buildings paints, play dough, lipstick and oil pastel.

Methods: Three samples from each oil paints and plastic paints for buildings (the most common paint in use); the 
most available, oil pastel (one sample); the most common, play dough (one sample); and four random lipsticks were evaluated. Graphite furnace atomic absorption spectrometry (Perkin Elmer, Model 3030 with HGA 400 Programmer) for determination of lead concentrations was used.

Results: Lead concentrations are summarized in the table:

\begin{tabular}{|c|c|c|c|c|c|}
\hline Mean & $\mathrm{S} 4$ & S3 & $\mathrm{S} 2$ & S1 & \\
\hline 14,010 & & 730 & 25,050 & 16,260 & Oil paint (ppm) \\
\hline \multirow[t]{3}{*}{758} & & 2,275 & 0.12 & 0.50 & Plastic paint (ppm) \\
\hline & & & & 4.5 & Oil pastel (ppm) \\
\hline & & & & 0.70 & Playdough (ppm) \\
\hline 0.77 & 0.4 & 1.67 & 0.55 & 0.60 & Lipstick (ppm) \\
\hline
\end{tabular}

Conclusion: Comparing these results with the recommended maximum lead levels in buildings paints $(0.06 \mathrm{ppm})$ and something that may be consumed frequently by small children $(0.1 \mathrm{ppm})$ and according to UNEP, FDA, CSFAN articles, these measurements are much higher.

Recommendation: Enforcing standard limits in building paints, oil pastel and play dough are recommended.

\section{(23) Designing a Software for Identifying Iranian Medications According to Their Appearance}

Reza Afshari, Seyed Mohammad Reza Mohajeri, Seyed Amir Reza Mohajeri.

Medical Toxicology Research Center, Department of Internal Medicine, Imam Reza Hospital, Mashhad, Iran

Introduction: Different branches of medicine cover a limited number of common or rare diseases. Medical toxicology, however, includes a wide range of diseases due to a variety of available medications and poisons. As a result, it is not possible to memorize all possible related syndromes or diseases. High-income countries including USA, UK and New Zealand have developed some software (TOXNET, TOXBASE and TOXZIN) to tackle this problem. This project is willing to produce software for electronically recognizing Iranian medications. The applied goal of this study is to facilitate diagnosis and increase precision of medical activity in emergency and toxicology departments.

Materials and Methods: During a 10-month period and via using Microsoft Visual Basic.Net (2008), 4-GB software including pictures and texts has been developed to record related information to Iranian medications. Additionally, these medications were photographed and uploaded in the software. Recorded information and pictures were linked to each other. In addition, physical characteristics of 994 Iranian medications were recorded and merged with related data to facilitate the diagnosis.
Results: By using the powerful search tools of this software it is now possible to recognize 994 Iranian medications according to their color, size, shape, type and other individual characteristics. Any particular selection will lead to graphical as well as textual information related to each medication.

Conclusion: Via using this software, cure of poison patients is easily available for the first time for Iranian medications.

\section{(24) Clinical Characteristics of Kratom Abusers in Thailand}

Satariya Trakulsrichai ${ }^{1}$, Winai Wananukul ${ }^{1}$, Umaporn Udomsubpayakul $^{2}$, Rajarek Junsang ${ }^{3}$.

${ }^{I}$ Ramthibodi Poison Center, Faculty of Medicine, Ramathibodi Hospital, Mahidol University, Bangkok, 10400 Thailand.

${ }^{2}$ Section for Clinical Epidemiology and Biostatistics, Research Center, Faculty of Medicine, Ramathibodi Hospital, Mahidol University, Bangkok, 10400, Thailand.

${ }^{3}$ Emergency Department, Faculty of Medicine, Ramathibodi Hospital, Mahidol University, Bangkok, 10400, Thailand.

Introduction: Mitragyna speciosa Korth, or Kratom in Thai, is a native tree indigenous to Southeast Asia; especially in Thailand, it is traditionally used as a folk medicine for centuries. Kratom is misused as a drug of abuse, becomes wildly spread legally to many countries and is easily available through online markets or some shops. Nowadays, the scientific and clinical studies of Kratom are rather limited, particularly the clinical data from chronic abusers.

Objectives: The objective of this study is to collect and analyze the clinical characteristics data of Kratom abusers in Thailand.

Methods: We carried out a prospective study by interview, review and analyze the questionnaires data from Kratom abusers.

Results: There were totally 26 Kratom abused cases. All were male. The cases were from the central region including Bangkok $(80.77 \%)$ and the south of Thailand $(19.23 \%)$. The average age was 48.6 years. Most were agriculturists $(42.31 \%)$. Fifty percent of the cases did not graduate primary school. The median income was $6,000 \mathrm{baht} / \mathrm{month}$. The median abused duration was 10 years (the maximum 40 years). All ate leaves and did not use other substances of abuses. The most common method the cases used was chewing and swallowing whole fresh leaves $(76.92 \%)$. The average amount was 16.4 leaves/day. The most common reason for starting the use of Kratom was to physically endure the hard work under the sun and to relieve fatigue $(76.92 \%)$. Of the cases, $76.9 \%$ used to use Kratom to treat some illnesses, of which diarrhea was the most common. Some used Kratom to substitute for heroin, alcohol and amphetamine, and they were successful to rid of these addictions. When the cases ate more than usual, they reported that dizziness was the most 
common symptom ( $30.77 \%$ ). The most common symptoms after they stop eating Kratom (withdrawal symptoms) were myalgia (76.92\%), yawning (53.85\%) and jerking $(46.15 \%)$. Of the cases, $84.6 \%$ did not use any method to relieve this withdrawal symptom. All reported that there was no visit or admission to the hospital related to Kratom. Of the cases, $80.76 \%$ reported that they did not have side effects from Kratom. Most did not have any weight and skin color changes during the abused period.

Conclusions: All cases in this study were chronic Kratom abusers. The most common symptoms when the cases ate more and stopped eating were dizziness and myalgia, respectively. There were no serious withdrawal or toxicity symptoms reported. Most had no side effect from Kratom despite prolonged use. Some used Kratom as the remedy for heroin, alcohol and amphetamine withdrawal.

(25) Evaluation of the Incidence, Symptoms, Signs, Prognosis, and Treatment of Stimulant Abuser Patients Referred to Poisoning Ward of Shoushtari Hospital in Shiraz from September 2007 to September 2009

F.goudarzi, H. Nasari, Z. Hossaini.

Department of Clinical Toxicology, Shiraz University of Medical Sciences, Shiraz, Iran

Objective: Amphetamine and methamphetamine use among young people is of significant social, economic and public health concern to affected communities and policy makers. The aim of this study is to identify the epidemiology of poisonings with these agents in Shiraz population.

Methods: This was a cross-sectional study being performed in Shoushtari Hospital during a 24-month period from September 2008 to 2010 . We included all the patients who referred to our center with acute poisoning and were diagnosed to have amphetamine and other neuro-stimulator agents poisoning. The demographic information as well as type of consumed toxin, route of consumption, vital signs, clinical signs and the outcome was recorded using a standard questionnaire. Data were analyzed using SPSS software.

Results: Overall, 9,563 patients with acute poisonings referred to our center out of which 544 (5.68\%) were neurostimulator agents' poisonings. Out of these, 439 (4.59\%) were male and $105(1.1 \%)$ were female. Crystal was the most common toxic agent being reported in $46.5 \%$ of the patients. Inhalation was the most common route of consumption being found in $62.9 \%$ of the patients. Crack, cocaine and crystal consumption was associated with male gender while Ritaline and ecstasy consumption were more common among females. Hallucinations were significantly higher in women $(p=0.001)$ while dyspnea was more common among men $(p<0.05)$. Complete recovery was associated with younger age $(p<$ $0.05)$. Generally, the most common and dangerous symptoms and signs of the patients were: agitation, chest pain, headache, tachycardia and high blood pressure, respectively.

Conclusion: Inhalation was the most common route of consumption which is due to its easy application. The prevalence of inhalation and injection was lower in women compared to men while those agents that can be easily consumed including Ritalin and ecstasy were more common among women. Consumption of these drugs is considered a medical emergency which should be treated aggressively.

\section{(26) Prognostic Factors in Mushroom Poisoning}

Dadpour B, Rajabi M, Afshari R.

Imam Reza Hospital, Mashhad University of Medical Science, Mashhad, Iran

Introduction: There are several types of toxic mushrooms in all over the world. Almost all of mushroom fatalities are associated with cyclopeptide-containing species, and the amatoxins are the most toxic of the cyclopeptides. Clinical manifestations following ingestion of toxic mushrooms are primarily gastrointestinal symptoms and this phase sometimes leads to hepatic, renal and nervous system damage and even death after a transient improvement phase. In this study, we aimed to determine prognostic factors in the patients with mushroom poisoning.

Methods: We studied records of all of admitted patients with mushroom poisoning in Clinical Toxicology Centre of Mashhad from February 2006 to February 2011(during 5 years) and our findings were analysed by SPSS software. Results: Among 32 admitted patients: $59 \%$ were discharged with recovery, $18 \%$ needed to mechanical ventilation along with progression of their symptoms, $6 \%$ needed to ICU admission, $19 \%$ were self-discharged and $22 \%$ expired. The mean (SD, min-max) of: serum glutamic-oxaloacetic transaminase (SGOT) is $434(947,15-3,812)$, serum glutamic pyruvic transaminase (SGPT) is $534(972,10-3,426)$, Bil (total) is $4(6.3,0.3-27.9)$, Bil (direct) is $1.7(2.5,0.1-9.3)$, PT is 24.6 (24.6, 11.9-121), PTT is $33.8(12.6,19.3-72.6)$ and INR is 2.5 (3.6, 0.9-15). There was significant relationship between the need to mechanical ventilation and rise of INR $(P=0.035)$, rise of PT $(P=0.006)$ and rise of PTT $(P=0.014)$. Also, there was significant relationship between expire rate and rise of INR $(P=0.035)$, rise of PT $(P=0.011)$ and rise of PTT $(P=0.003)$. There was significant relationship between need to ICU admission and rise of INR $(P<0.001)$. We did not find any significant relationship between ICU admission rate, need to mechanical ventilation and expire rate with rise of hepatic transaminases and bilirubin of serum.

Conclusion: Impairment of coagulation tests (PT, INR and PTT) is a poor prognostic factor in mushroom poisoning whereas transaminases (SGOT and SGPT) rise does not have any role in prognosis of these poisoned patients. 


\section{(27) Comparison of Therapeutic Effects of Garlic and D-Penicillamine in Patients with Chronic Occupational Lead Poisoning}

Sina Kianoush, Mahdi Balali-Mood, Reza Mousavi, Valiollah Moradi, Mahmoud Sadeghi, Omid Rajabi, Mohammad Taghi Shakeri.

\section{Mashhad University of Medical Sciences, Mashhad, Iran}

Previous studies on animals have revealed that garlic (Allium sativum) is effective in reducing blood and tissue lead concentrations. The aim of this study was to investigate therapeutic effects of garlic and compare it with $D$-penicillamine in patients with chronic occupational lead poisoning. After coordination and obtaining informed consent, clinical examinations and blood lead concentration (BLC) of 117 workers at a car battery industry were investigated. BLC was determined by heated graphite atomization technique of an atomic absorption spectrophotometer. The workers were randomly assigned into two groups of garlic $(1,200 \mu \mathrm{g}$ allicin, three times daily) and $D$-penicillamine ( $250 \mathrm{mg}$, three times daily) and treated for 4 weeks. BLC was determined again 10 days post-treatment. Clinical signs and symptoms of lead poisoning were also investigated and compared with the initial findings. Clinical improvement was significant $(P<0.031)$ in a number of psycho-neuromuscular manifestations (irritability, headache and decreased deep tendon reflexes) after reatment by garlic but not $D$-penicillamine. BLCs were reduced significantly $(P=0.002$ and $P=0.025)$ from $426.32 \pm 185.128$ to $347.34 \pm 121.056 \mu \mathrm{g} / \mathrm{L}$ and from $417.47 \pm 192.54$ to $315.76 \pm$ $140.00 \mu \mathrm{g} / \mathrm{L}$ in garlic and $D$-penicillamine groups, respectively, with no significant difference $(P=0.892)$ between them. The severity of side effects was significantly $(P=0.023)$ higher in $D$-penicillamine than in garlic group. Thus, garlic seems safer and more effective clinically than $D$-penicillamine in mild to moderate chronic occupational lead poisoning. However, further detailed investigations on different populations with lead poisoning are required to make a general conclusion.

\section{(28) Searching the Mechanisms of Ethylene Glycol Renal Toxicity_Preliminary Results}

Knut Erik Hovda ${ }^{1}$, Sigurd Boye ${ }^{2}$, Dag Jacobsen ${ }^{3}$, Kenneth McMartin ${ }^{4}$.

${ }^{I}$ The Norwegian NBC Center, Department of Acute Medicine, Oslo University Hospital, Ullevaal, Oslo, Norway.

${ }^{2}$ Clinical and Biomedical Engineering, Oslo University Hospital, Ullevaal, Oslo, Norway.

${ }^{3}$ Department of Acute Medicine, Oslo University Hospital, Ullevaal, Oslo, Norway;

${ }^{4}$ Department of Pharmacology, Toxicology and Neuroscience, Louisiana State University Health Sciences Center, Shreveport, LA, USA
Introduction: The renal toxicity found in ethylene glycol (EG) is predominantly through the creation of calcium oxalate monohydrate $(\mathrm{COM})$ crystals, but the final mechanisms are not understood and various mechanisms have been proposed. Our aim was to search for crystals not only inside the cells, but possibly inside cell organelles, such as the mitochondria, to further understand the toxic mechanisms.

Methods: Human renal proximal tubule cells were grown to confluency on coverslips, and then treated with COM at different concentrations and for different time intervals. The sample was fixed using Karnovsky's fixative, postfixed, dehydrated and embedded in araldite 502 using microwave procedure. Ultra-thin sections were examined in a 120 kV/LaB6 Tecnai G2 Spirit BioTWIN transmission electron microscope and images were sampled using an Eagle $4 \mathrm{k}$ CCD camera.

Results: Crystals were found inside vacuoles with degradation of segregated cellular components. We also found clear evidence of needle-shaped crystals inside the mitochondrion. Chromatin condensation, DNA fragmentation, blebbing and cell shrinkage were also observed.

Discussion: The present results show for the first time the existence of the COM-crystals in ethylene glycol poisonings inside the mitochondria, thus supporting theories of involvement of these organelles. The transport to, or the formation of, these crystals inside the mitochondria needs further studies, as well as the mechanisms for toxicity. However, the chromatin condensation, DNA fragmentation, blebbing and cell shrinkage are all characteristic of apoptotic cell death. Finding the mode of transport of crystals inside the mitochondria (either by endocytosis, active transporting mechanisms of the crystals or transporters for the ions) and the mechanisms of cell death, open a possibility for design of new treatment modalities in the late stages of EG poisonings.

\section{(29) Management of Severe Eye Injury from Paraquat}

Ravindra Fernando ${ }^{1,2}$, Shiranthi Perera ${ }^{1,2}$.

${ }^{1}$ Department of Forensic Medicine and Toxicology, Faculty of Medicine, University of Colombo, Colombo, Sri Lanka.

${ }^{2}$ Police Hospital, Colombo, Sri Lanka

Paraquat eye injury is rare. We report a case of severe eye injury from paraquat to emphasize the need for proper and timely management. A 32-year-old lady was admitted with acute paraquat ingestion. While she was drinking paraquat from a full bottle, her husband attempted to grab the bottle, resulting in paraquat splashing on to her left eye. The eye was washed immediately but she developed irritation, burning sensation and pain which became severe over the next few days. When she presented on the fourth day, she had ptosis and complained of poor vision, severe pain and 
difficulty in opening the eye. Her visual acuity was confined to hand movements. The conjunctiva was heavily oedematous and hyperaemic. Fluorescein staining revealed a large epithelial defect covering almost entire cornea. The anterior chamber showed a low-grade uveitis with +2 cells. Intra ocular pressures in both eyes were similar. The eye was patched for $24 \mathrm{~h}$ after instilling fucithalmic eye ointment. When examined after $24 \mathrm{~h}$, her pain and conjunctival hyperaemia were less. The epithelial defect showed superficial healing. An area of limbal ischaemia of less than $180^{\circ}$ was visible (grade 2 chemical injury). A chemical injury therapeutic regime was commenced. Artificial tears (Carboxymethyl cellulose $0.5 \%$ ), ciprofloxacin eye drops, tetracycline eye ointment, prednisolone acetate $1 \%$ eye drops and vitamin C $1,500 \mathrm{mg}$ daily were commenced. She was examined regularly and the treatment was altered in the next few days. On the fifth day of treatment, she had no ptosis, no pain, the conjunctiva was minimally hyperaemic and the limbal ischaemia had reduced. On the 12th day the visual acuity of the eye was $6 / 6$. There were no signs of eye injury. Timely and appropriate intervention can bring excellent recovery from paraquat eye injury as shown in this case.

\section{(30) The Changing Patterns of the Epidemiology of Acute Self-Poisoning in Rural Sri Lanka}

Lalith Senarathna $^{1,2}$, Shaluka F Jayamanna ${ }^{2}$, Patrick Kelly ${ }^{1}$, Nicholas A Buckley ${ }^{3}$, Michael J Dibley ${ }^{1}$, Andrew H Dawson ${ }^{2,3}$. ${ }^{1}$ South Asian Clinical Toxicology Research Collaboration, Faculty of Medicine, University of Peradeniya, Peradeniya, Sri Lanka.

${ }^{2}$ Sydney School of Public Health, University of Sydney, Sydney, NSW, Australia.

${ }^{3}$ Department of Clinical Medicine, Faculty of Medicine, University of Kelaniya, Kelaniya, Sri Lanka.

${ }^{4}$ Professorial Medicine Unit, POW Clinical School, University of New South Wales, Sydney, NSW, Australia.

${ }^{5}$ Royal Prince Alfred Clinical School, University of Sydney, Sydney, NSW, Australia

Introduction: Acute poisoning is a major public health issue in many parts of the world. The epidemiology and the mortality rate are different from low- and middle-income countries, including Sri Lanka, to high-income countries. The aim of this study was to provide details about the epidemiology of acute poisoning in a rural Sri Lankan district and to identify the changing patterns and epidemiology of poisoning.

Methods: A prospective study was conducted from September 2008 to January 2010 in all hospitals with inpatient facilities in Anuradhapura district of North Central Province of Sri Lanka. Acute poisoning data was extracted using structured queries from patient charts.
Results: There were 3,813 poisoned patients admitted to all the hospitals in Anuradhapura district during the study period giving an annual age-adjusted population incidence of 459 poisoning cases per 100,000 of population. Although the number of male and female patient were approximately similar, the age distribution differed by gender. More young patients, particularly a larger proportion of females, below age 20 were admitted to these hospitals. Although, pesticides ranked as the most common type of poison, the number of medicinal drug poisonings has increased significantly since 2005 in this area.

Conclusions: The incidence of acute poisoning remains high in this rural setting in Sri Lanka, and the most vulnerable group are young females. Medicinal drug poisoning has increased significantly over last 5 years. This trend indicates that poisoning treatment protocols and guidelines should be adjusted to take account of these changes, and the need for strategies to reduce higher incidences among young population by targeting these groups in focused interventions.

\section{(31) Prescription-Related Problems of Non-Steroidal Anti-Inflammatory Drugs in a Primary Care Setting}

Abdullah AH Dhabali, Rahmat Awang, Zulham Hamdan, Sa'ed H. Zyoud.

Universiti Sains Malaysia, Penang, Malaysia

Background: Non-steroidal anti-inflammatory drugs (NSAIDs) are frequently used in the treatment of different painful conditions. Their use is increasing due to the increased incidence of rheumatic diseases. Although generally well tolerated, these drugs are associated with serious adverse reactions such as upper gastrointestinal ulcers and bleeding, hypersensitivity reactions and renal failure. NSAIDs use can improve the quality of life for patients, but may also result in adverse health outcomes if used inappropriately. NSAIDs prescription-related problems (NSAIDs PRPs) can cause considerable morbidity and mortality, and may lead to hospital admission.

Purpose: The objectives of this study are (1) to obtain information regarding the prevalence of NSAIDs PRPs and (2) to determine the demographic and clinical characteristic of patients exposed to these problems.

Methods: We retrospectively collected data from one academic year using computerised databases at the Universiti Sains Malaysia (USM) from patients of USM's primary care. Lists of NSAIDs PRPs were developed from authentic clinical pharmacy and drug information sources. Multivariate regression was used to determine the characteristics of patients exposed to NSAIDs PRPs.

Results: During the study period, 12,622 of NSAID items were Prescribed for 6,518 patients (mean $\pm \mathrm{SD}=1.94 \pm 1.90$ ). This represented a prevalence of $36 \%$. Five hundred and ninety one NSAIDs PRPs were identified in a cohort of 434 
patients, representing an exposure prevalence of $6.7 \%$ of all NSAID users. Multivariate logistic regression analysis revealed that patients being Malay, being a staff, having multiple prescriptions and having more comorbid conditions were more likely to be exposed to NSAIDs PRPs.

Conclusions: This is the first study in Malaysia that presents data on the prevalence of NSAIDs PRPs. Exposure to these problems is associated with specific sociodemographic and health status factors. These results should help to raise the awareness of clinicians about serious NSAIDs PRPs.

\section{(32) Drug-Facilitated Crime in Tehran, Iran}

Hossein Hassanian-Moghaddam ${ }^{1}$, Fatemeh Gharedaghi ${ }^{2}$, Maryam Akhgari ${ }^{3}$, Fakhreddin Taghaddosinejad ${ }^{2}$.

${ }^{1}$ Department of Clinical Toxicology, Shahid Beheshti University of Medical Sciences, Tehran, Iran.

${ }^{2}$ Department of Forensic Medicine, Tehran University of Medical Sciences.

${ }^{3}$ Forensic Toxicology Department, Scientific and Educational Research Center of Legal Medicine Organization (LMO), Tehran, Iran

Background and Objectives: The objective of this study is to investigate claimed drug-facilitated crime (DFC) in the patients who had been hospitalized in two referral hospitals in Tehran, Iran.

Materials and Methods: All hospitalized patients who were referred on February 2010 to March 2011 and suspected/claimed DFC were included. Those who had taken unknown drugs in the last week before admission or diagnosis of any other cause of poisoning by accident, deliberate self-poisoning or drug abuse were excluded. Toxicological analyses were done after collecting $20-\mathrm{ml}$ urine sample on arrival time using gas chromatography-mass spectrometry.

Results: A total of 53 victims were included. Mean age was $31.6 \pm 12.4$ years (range 6-75), mostly males. The victims were predominantly workers $(41.5 \%)$, from Tehran, with 12 years of schooling ( $35.8 \%$ diploma), who accepted drinking juice $(35.8 \%)$,when commuting inside or intercity $(37.9 \%)$, were stolen thereafter $(77.4 \%)$ and brought to emergency department by EMS ambulances $(39.6 \%$ ) while sleepy $(56.6 \%)$ upon arrival. After full consciousness, majority remembered a male gender of offender $(77.4 \%)$, who was a stranger $(75.5 \%)$ with estimated age range of $22-$ 52 years. Those who noticed significant taste change of potables and edibles were admitted earlier $(p<0.05)$. Thirty eight $(71.7 \%)$ victims remembered estimated time of intoxication. Of whom, $92.1 \%$ were admitted before $24 \mathrm{~h}$ post-ingestion (range $1-120 \mathrm{~h}$, median $11 \mathrm{~h}$ ). Two car accidents happened in victims, who were drivers, with no major trauma. Among 53 urine samples analyzed, 10
(18.9 \%) were positive for metoclopramide, dextromethorphan, $6(11.4 \%)$ for tramadol, 5 (9.4\%) for theobromine, $4(7.5 \%)$ for lorazepam, $3(5.7 \%)$ for nortetrazepam, codeine, methadone, 2 (3.8\%) for promethazine and 1 (1.9\%) for cypreheptadine, morphine, adduct, carbamazepine and levorphanol. In nine patients, no positive results were obtained.

Conclusion: Our findings show that opioids, benzodiazepines and antihistamines are the most drugs used solely or together in DFC. Metoclopramide as an antiemetic may be used to prevent nausea and vomiting in criminal poisoning to achieve more DFC effects.

\section{Poster Presentations}

\section{(33) Bhopal Gas Tragedy_Worst Chemical Disaster}

Anand Tiwari, Nisanth Nambian.

Government Medical College, Bhopal, Madhya Pradesh, India

Bhopal is very much infamous for worst chemical disaster which occurred on night of 2/3 December 1984. Bhopal is situated in central India and is capital of Madhya Pradesh with population of 1,792,203 (2011). The chemical responsible for the accident was methyle isocynate or MIC (other names are: isocyanatomethane and methyl carbylamine).

Chemical Formula: $\mathrm{H}_{3} \mathrm{C}-\mathrm{N}=\mathrm{C}=\mathrm{O}$.

Physical/Chemical Properties: Methyl isocyanate (MIC) is a clear, colorless, lachrymatory, sharp-smelling liquid. It is highly flammable, boils at $39.1{ }^{\circ} \mathrm{C}$. Methyl isocyanate is soluble in water to 6-10 parts per 100 parts, but it reacts with the water. Methyl isocyanate reacts readily with many substances that contain $\mathrm{N}-\mathrm{H}$ or $\mathrm{O}-\mathrm{H}$ groups and some other compounds. It also reacts with itself to form a trimer or higher molecular weight polymers. Methyl isocyanate reacts with water to form 1,3-dimethylurea and carbon dioxide with the evolution of heat ( 325 calories per gram of MIC that reacts).

Morbidity/Mortality: The government of Madhya Pradesh has confirmed a total of 3,787 deaths. Half a million people were exposed to the gas and 25,000 have died to date as a result of their exposure. More than 120,000 people still suffer from ailments caused by the accident and the subsequent pollution at the plant site.

Present Sufferings: Breathlessness, persistent cough, diminished vision, early age cataracts, loss of appetite, menstrual irregularities, recurrent fever, back and body aches, loss of sensation in the limbs, fatigue, weakness, anxiety and depression are the most common symptoms among survivors. Furthermore, there is an alarming rise in cancers, TB and reproductive health problems. The official agency for monitoring deaths has been closed since 1992. 
Ongoing Contamination: Contamination at the site and surrounding area was not caused by the gas leakage. The area around the plant was used as a dumping ground for hazardous chemicals. So water in 100 wells is unfit for drinking. A number of toxins, including mercury, lead, 1,3,5 trichlorobenzene, dichloromethane and chloroform, is in nursing women's breast milk. Mercury levels are at "20,000 and 6 million times" higher than expected levels; heavy metals and organochlorines were present in the soil. Chemicals that have been linked to various forms of cancer were also discovered, as well as trichloroethylene, known to impair fetal development, at 50 times above safety limits.

(34) Detection of Contaminants in Food Products and Health Supplements: Findings by the Toxicology Laboratory of the National Poison Centre of Malaysia in 2010

Norjuliana M.N., Che Nin M., Razak.L.

National Poison Centre, Universiti Sains Malaysia, Penang, Malaysia

Each year, the toxicology laboratory at the National Poison Centre of Malaysia receives numerous samples for general drug screening or toxicological analysis. The laboratory provides 14 types of toxicological testing which include determination of contaminants or adulterants in food products and health supplements. The lab also conducts test to determine heavy metals, natural chemical compounds in plants and marine toxin. Samples received by the lab varies from herbal preparations to cosmetic, chemicals, pharmaceuticals, human biological fluids, foods and other consumer products. The clients are mainly manufacturers and suppliers of food products and health supplements. Occasionally, the lab may receive samples from private individual, clinics, hospitals, government agencies and other laboratories. In 2010, a total of 879 samples from 148 clients were analysed. These include 452 research samples and 427 non-research samples. Of the non-research samples tested, $43.8 \%$ were found positive with 'dangerous or forbidden substances'. Many consumer products were either found contaminated or adulterated with drugs $(94.7 \%)$, pesticides $(4.3 \%)$ and natural plant toxin (1\%). Male sexual enhancement drugs (sildenafil and analogues) were the main drugs found in food, health supplement and herbs (40.1\%) followed by slimming agent drugs (sibutramine and analogues; $20.9 \%)$. Other drugs detected in samples $(34.2 \%)$ were identified as non-steroidal anti-inflammatory drugs (indomethacin, ibuprofen and salicylate-34.8\%), corticosteroids (dexamethasone, betamethasone and cortisone-17.45\%), stimulants (caffeine-11.6\%) and antihistamine (chlorpheniramine-10.1\%). A number of other products were contaminated with pesticides residues $(4.8 \%)$ which include permethrin, DDT metabolites, naphthalene, methomyl, chlorpyrifos, hexaconazole and chlorothalonil. Advanced technology has contributed to the production of many new synthetic drugs and chemicals. It is vital for regulatory bodies to carry out more checks and inspections to curb the number of illegal consumer health products.

(35) Dental Treatment Needs of Iranian Veterans with Sulfur Mustard Poisoning Referred from Imam Reza Hospital Medical Toxicology Research Center to Oral Medicine Department of Mashhad University of Medical Sciences

Javad Sarabadani ${ }^{1}$, Atessa Pakfetrat ${ }^{1}$, Amir Moeentaghavi ${ }^{2}$, Mahdi Balali-Mood ${ }^{3}$, Hasan Hosein pour Jajarm ${ }^{1}$, Mansour Naseri ${ }^{3}$.

${ }^{1}$ Assistant professor, Department of Oral Medicine, Faculty of Dentistry and Dental Research Center, Mashhad University of Medical Sciences, Mashhad, Iran.

${ }^{2}$ Associate professor, Department of Oral Medicine, Faculty of Dentistry and Dental Research Center, Mashhad University of Medical Sciences, Mashhad, Iran.

${ }^{3}$ Associate professor, Department of Periodontology, Faculty of Dentistry and Dental Research Center, Mashhad University of Medical Sciences, Mashhad, Iran.

${ }^{4}$ Professor of Medicine and Clinical Toxicology, Director of Medical Toxicology Research Center, Imam Reza Hospital, Mashhad University of Medical Sciences, Mashhad, Iran ${ }^{5}$ Dentist

Introduction: The effects of chemical gas on tissue exposed to environment (skin, airways, and eyes) depends on the amount of poison and duration of contact, and some areas such as skin folds and parts with thin epidermis are more sensitive to gas. The aim of this study was determining the therapeutic needs of Iranian veterans with sulfur mustard poisoning referred from Imam Reza Hospital Medical Toxicology Research Center to Oral Medicine Department of Mashhad University of Medical Sciences.

Methods: In this descriptive cross-sectional study, 40 of the sulfur mustard veterans referred from Imam Reza Hospital Medical Toxicology Center to Oral Medicine Department of Mashhad University of Medical Sciences were orally examined in 2009. The patients' data were recorded on a WHO oral health assessment form (1997). The statistical analysis was performed using SPSS program.

Results: Among the 40 sulfur mustard veterans, $75 \%$ patients need to be treated dentally. One fourth of the subjects had complete removable denture and the other subjects need dental treatment. The most common therapeutic needs were oral hygiene instruction, partial removable prosthesis, and fixed prosthesis, respectively.

Conclusion: Most patients need treatment. According to periodontal and oral health indexes, almost all patients had low oral hygiene level and periodontal involvement to some extent. 
Presence of highly frequent oral soft tissue lesions in Iranian veterans with sulfur mustard poisoning compared to general population and according to dental need treatment necessitates periodical oral soft tissue and routine oral dental examinations by oral medicine specialists and treatment more carefully.

\section{(36) Hemolytic Anemia Presenting as Hemoglobinuria from Paradichlorobenzene Ingestion}

Mary Ondinee U. Manalo ${ }^{1}$, Cherry Grace G. Quingking ${ }^{2}$, Clarissa Paz Dioquino ${ }^{2,3}$.

${ }^{1}$ Department of Medicine, University of the PhilippinesPhilippine General Hospital, Manila, Philippines.

${ }^{2}$ National Poison Control Center, University of the Philippines-Philippine General Hospital, Manila, Philippines.

${ }^{3}$ Section of Neurology, Department of Neurosciences, University of the Philippines-Philippine General Hospital, Manila, Philippines

Synopsis: A 24-year-old man was diagnosed with hemolytic anemia when he presented with hemoglobinuria, low hemoglobin, and increased bilirubins, after ingesting mothballs containing paradichlorobenzene. He was transfused with 5 units of PRBC and was discharged improved.

Clinical Presentation: A 24-year-old man intentionally ingested three crushed mothballs. Five days after ingestion, the patient manifested with anemia and bright red urine.

Physical Findings: The patient was received stable at the Emergency Room of the Philippine General Hospital with coffee-ground vomitus, pink palpebrals, icteric sclerae, epigastric tenderness, and normal-sized liver.

Laboratory Work-up: The content of the mothball was established to be paradichlorobenzene using the floatation test. The patient tested positive for methemoglobinemia using the filter paper test. Initial hemoglobin was $120 \mathrm{~g} / \mathrm{L}$ that markedly decreased to $53 \mathrm{~g} / \mathrm{L}$ on the third hospital day with subsequent indirect bilirubinemia. Peripheral blood smear revealed normocytic, normochromic anemia with slight poikilocytosis and no evidence of toxic granulations. LDH peaked to $1,118 \mathrm{U} / \mathrm{L}$. Urine dipstick tested positive for hemoglobin. Coomb's test was negative.

Diagnosis: The patient was diagnosed to have hemolytic anemia based on a marked decrease in hemoglobin, hemoglobinuria, indirect bilirubinemia, and supporting literature that paradichlorobenzene indeed causes hemolytic anemia.

Treatment: Patient was given high-flow oxygen and ascorbic acid for his methemoglobinemia. Hemolytic anemia was managed supportively by blood transfusion.

Outcome: The patient was discharged improved after transfusion of 5 units of PRBC.

Significance: Hemolytic anemia is a rare, and often, a delayed complication of paradichlorobenzene ingestion and inhalation. Anticipatory care is therefore of utmost importance. To date, this is the first reported case of hemolytic anemia, presenting initially as hemoglobinuria, from mothball ingestion in the Philippines. Recommendations: This report will be submitted to the Department of Health, the Bureau of Food and Drugs, and the Fertilizer and Pesticide Authority with a recommendation to impose appropriate warnings on all products that contain paradichlorobenzene.

(37) Poisoning in Children: a 4-Year Review (2006-2009) on Cases Reported to the National Poison Centre of Malaysia

Nur Afni A, Rahmat A, Sa'ed Z, Sulastri S, Haslina H, Sazaroni MR.

National Poison Centre, Universiti Sains Malaysia, Penang, Malaysia

Objective: Poisoning involving children continues to represent the major poisoning emergency reported to the National Poison Centre (NPC). A better understanding of the pattern of acute poisoning in children is an important preventive strategy. The aim of this study is to elucidate the prevailing trend of acute poisoning in children based on the poisoning cases reported to the NPC.

Methods: Cases of acute poisoning involving children reported to the NPC between 1 January 2006 and 31 December 2009 were reviewed. Paediatric age groups were classified into four categories according to the IPCS-INTOX Data Management System (1 day- 4 weeks: neonates; 4 weeks-12 months: infants; $1-4$ years: toddlers; and 5-14 years: children). Age, date of exposure, reason of exposure, and type of substance involved in the exposure were evaluated. SPSS version 15 was used for descriptive analysis of the data collected.

Results: A total of 2,468 calls on poisoning involving children exposures were received, making up about $26.2 \%$ of the total cases of poisoning during the study period. Of the total poisoning cases involving children, $1,593(60.1 \%)$ were in the toddler age group. Most calls were made by medical doctors 2,445(99.0\%). The number of poisoning calls was found to have significantly increased yearly from 385 calls $(16 \%)$ in 2006 to 902 calls $(37 \%)$ in $2009(\mathrm{p}<0.001)$. The largest contributing factors in children poisoning was pharmaceutical substances, household chemicals and pesticide, which accounted for 968 (39.2\%), 906 (36.7\%), and 440 (17.8\%) cases, respectively. Majority of the exposures occurred at home 233 (94.6\%), through oral ingestion 2,432 (98.5\%), and were due to accidental poisoning 2,107 (85.3\%). Information on the patient's outcome was not available.

Conclusion and Recommendation: The increased number in poisoning calls involving children is alarming and should be highlighted at the national level for preventive strategy to be established. Since cases handled by the NPC constitutes a proportion of all poisoning cases handled by the emergency 
department, a national study on the magnitude of poisoning with respect to the household chemicals and medication use should be carried out.

(38) Postmortem Cancer Registry in Deaths with a History of Exposure with Chemical Warfare Agents (CWA)

Ghasempouri S Kh ${ }^{1}$, Mohebati $\mathrm{H}^{2}$, Afshari $\mathrm{R}^{1}$, Rahimi R. H. ${ }^{3}$, Khosrojerdi $\mathrm{H}^{1}$.

${ }^{1}$ Medical Toxicology Research Center, Medical Toxicology

Center, University of Medical Sciences, Mashhad, Iran.

${ }^{2}$ Legal Medicine Organization, Mashhad Iran.

${ }^{3}$ Departmant of New Sciences and Technology Medical School, Mashhad, Iran

Introduction: Chemical warfare agents (CWA) were used in bombs during the World War (I). These agents were applied against Iranian veterans 20 years ago by Iraq. The purpose of this study was describing autopsy pathologic findings in those with a history of CWA exposure after two decades.

Methodology: A retrospective study designed to evaluate autopsy records of all those with a history CWA exposure from 2009 to 2010 in Mashhad catchment area with a population of 2,000,000.

Results: A total of 56 cases were evaluated ( $1 \%$ of all total reports). Mean (SD) age was 12.1 (5.5) years (36-84 years). Major cause of death was reported to be cardiopulmonary in 28 patients $(50 \%)$. In total, 11 cases $(19.6 \%)$ died due to cancer. Reported cancers were lung cancer, 4 (7.1\%); gastrointestinal, 3 (5.4\%); acute lymphoblastic leukemia, 3 $(5.4 \%)$; and a laryngeal cancer, $1(1.8 \%)$, which are relatively more common than in the general population.

Conclusion: This study revealed that deaths related to cancer are higher in cases with history of exposure to CWA. More drastic measures for these cases are recommended to evaluate presence of lung, gastrointestinal, and blood cancers in patients with history of exposure to CWA.

\section{(39) Trend of Poisoning Cases Referred to the National Poison Centre of Malaysia from 2006 to 2009}

Adilah M.A., Haslina H., Rahmat A., Sa'ed H.Z., Sulastri S. National Poison Centre, Universiti Sains Malaysia, Penang, Malaysia

Introduction: The National Poison Centre (NPC) of Malaysia provides 24-h Drug and Poison Information Service to health professionals and the general public. Most of the enquiries are related to poisoning cases, which were documented manually and electronically.

Objective: This study aimed to review and report the trend of poisoning cases handled by NPC for a period between 2006 and 2009.
Methods: In 2006, new classes of poisoning agents were introduced and certain classes were expanded into sub-classes based on the IPCS INTOX Data Management System. Age, gender, date of exposure, route of exposure, reason of exposure and type of poisoning were evaluated. Data were analysed using SPSS 18.0 to generate frequencies and percentages.

Results: A total of 9,413 poisoning cases were referred to NPC within the 4-year period with increased cases every year. Of the poisoning enquiries, $60.4 \%$ were handled after office hours and the majority ( $99 \%$ ) of enquiries received were from medical doctors. Of the cases, $59.6 \%$ involved adult patients and $56.3 \%$ were intentional incidents. Pharmaceutical products $(32.8 \%)$ were the main substances implicated, followed by pesticides $(32.2 \%)$ and household products $(23.8 \%)$. Analysis of the sub-classes of the common substances showed that herbicides, insecticides and cleaning agents were the most common substances implicated. The majority of the victims were male who were mainly involved in pesticides poisoning. Exposure to poisoning occurred mainly via ingestion (94\%) followed by inhalation (2.3\%).

Conclusion: The trend of poisoning incidences from 2006 to 2009 was almost similar to the previous 5-year report. Pesticides and pharmaceutical products remain the main substances implicated. The increase in the number of poisoning cases handled yearly implies that the 24-h Poisoning Call Service remains relevant and important especially to new medical officers.

(40) A Call for Caution of the Emergency Physician's Interpretation of the New Paracetamol Toxic Ingestion Dose Guideline - a Paediatric Case Study and Review

Ong Yong-Kwang Gene.

KK Women's and Children's Hospital, Little India, Singapore

Recent toxicology guidelines worldwide for paracetamol poisoning have raised the definition of a single, acute paediatric toxic ingestion of paracetamol from 150 to $200 \mathrm{mg} /$ kg. A 4-year-old Chinese girl presented with abdominal pain and vomiting was seen at the Kandang Kerbau Women's and Children's Hospital's Emergency Department in Singapore. It was disclosed during the consult that the child had consumed $178 \mathrm{mg} / \mathrm{kg}$ of paracetamol elixir $26 \mathrm{~h}$ prior while left unsupervised at home. The parents denied any further ingestion of paracetamol after the non-intentional exposure. However, intravenous $\mathrm{N}$-acetylcysteine was empirically started in view of the symptoms, and serum paracetamol level and liver enzyme assays were taken. A review of the records in the Emergency Department showed that the child was seen and discharged 2 days before for fever associated with a simple febrile seizure. She had taken three doses of $10 \mathrm{mg} / \mathrm{kg}$ (at six to seven hourly apart) of paracetamol elixir; with the last therapeutic dose $6 \mathrm{~h}$ prior to the non-intentional 
supratherapeutic ingestion (totaling $208 \mathrm{mg} / \mathrm{kg}$ within a 24$\mathrm{h}$ period of the supratherapeutic ingestion). Serum Paracetamol level was $6.3 \mathrm{mg} / \mathrm{L}$ at $26-\mathrm{h}$ post-supratherapeutic ingestion and aspartate (AST) and alanine (ALT) transaminases were 134 and $119 \mathrm{U} / \mathrm{L}$, respectively. The child was admitted for monitoring and recovered uneventfully without further sequelae after completing intravenous $\mathrm{N}$-acetylcysteine. For physician considerations of paracetamol toxicity, a thorough history of recent therapeutic ingestion of paracetamol should be carefully and actively sought when a supratherapeutic ingestion has occurred. It is also recommended that if a patient is symptomatic, intravenous $\mathrm{N}$ acetylcysteine should be initiated empirically irrespective of the history of estimated ingestion till biochemical investigations have conclusively excluded paracetamol toxicity.

\section{(41) Determination of Mercury in Fish Available} in Mashhad Market by Hydride Generation Atomic Absorption Spectrometry

Vali allah Moradi ${ }^{1,2}$, Mehrangiz Mokhtari ${ }^{1,2}$, Reza Afshari ${ }^{1,2}$, Seyed Reza Mousavi ${ }^{1,2}$, Maryam Vahabzadeh ${ }^{1,2}$, Mehdi Balali Mood ${ }^{1,2}$.

${ }^{1}$ Toxicology Laboratory of poisons Unit, Imam Reza Hospital, Faculty of Medicine, Mashhad University of Medical Sciences, Mashhad, Iran.

${ }^{2}$ Medical Toxicology Research Center, Faculty of Medicine, Mashhad University of Medical Sciences, Mashhad, Iran

Background: Fish is an excellent source of protein for human, but may contain mercury ( $\mathrm{Hg}$ ). It was thus aimed to determine $\mathrm{Hg}$ in fish of Mashhad markets by an atomic absorption spectrometry using mercury hydride system.

Methods: Before analysis, all laboratory equipment and containers were washed in $5 \% \mathrm{HNO} 3$ solution and rinsed with deionized water prior to each use. One gram of wet samples was weighted into quartz vessels, then added to $10 \mathrm{ml}$ of mixed acid solution $\mathrm{HNO}_{3}-\mathrm{H}_{2} \mathrm{SO}_{4}-\mathrm{HClO}_{3}-\mathrm{H}_{2} \mathrm{O}(5+2+2+2)$, mixed well, and put in boiling water bath for $1 \mathrm{~h}$. Digested samples were subsequently diluted with deionized water to $50 \mathrm{ml}$. Then the solution was filtered with filter paper (Whatman $150 \mathrm{~mm}$ ). A blank digest was carried out in the same way. Sodium borohydride was added to the sample solution to convert $\mathrm{Hg}^{2}$ ${ }^{+}$into elemental mercury $\left(\mathrm{Hg}^{0}\right)$. Mercuric hydride system of an atomic absorption spectrometer (Perkin Elmer model 3030 with MHS-10, Waltham, MA, USA) was used for analysis.

Results: Detection limit was $0.01 \mu \mathrm{g} / \mathrm{g}$, precision $3.11 \%$, accuracy $97.2 \%$, and recovery $93.0 \%$. The analyses were determined and repeated by using biological reference material (seronorm lot: 0903107). From six fish samples used for pilot study, maximum concentration of $\mathrm{Hg}$ was found in shark with $0.806 \mu \mathrm{g} / \mathrm{g}$ (PPM) and minimum in tigertooth croaker (Otolithes ruber) with $0.054 \mu \mathrm{g} / \mathrm{g}$.
Conclusions: Based on our findings, this extraction method and cold vapor generation atomic absorption spectrometry is a reliable method for estimation of fish mercury concentrations.

\section{(42) Cannabis Recreational Ingestion in North East Iran}

Heydari A, Afshari R.

Medical Toxicology Centre, Imam Reza Hospital, Mashhad University of Medical Sciences, Mashhad, Iran

Introduction: Acute illicit drug poisonings are relatively common in North East Iran. Locally processed cannabis, so called Maajon-Birjandi (MB), is being abused via ingestion. This study aimed at evaluating the clinical findings of these cases.

Methods: All cases referred to the toxicology triage, Imam Reza Hospital from Mashhad, Iran from 21 March 2009 to 21 February 2010 (11 months) with alleged MB were studied. A predesigned questionnaire was completed for each case.

Results: Fifty-three cases were included. Mean (SD, minmax) age was $20.8(3.2,14-73)$ years. Patients were dominantly male (96\%). Reduced level of consciousness and cognitive problems $(92 \%)$, drowsiness $(63 \%)$, tremor $(43 \%)$, ataxia (23\%) and hypotonia (21\%) were the most common findings. Tachycardia (heart rate more than $100 \mathrm{bpm}$ ) was present in $56 \%$. Other vital signs were within normal ranges for majority of cases. Urinary tetrahydrocannabinol was detected by urine screening tests. All cases were treated symptomatically and conservatively. Majority of cases recovered within 6 to $10 \mathrm{~h}$.

Conclusions: This study is reporting recreational cannabis ingestion from North East Iran for the first time. Symptoms are rather similar to cannabis smoking. It is plausible that half-life in cannabis ingestion could be longer.

\section{(43) Cephalexin-Induced Hemorrhagic Cystitis in a Child}

Pournasiri Z, Farnaghi F, Mehregan FF.

Shahid Beheshti Medical Science University

Hemorrhagic cystitis (HC) is an infectious or noninfectious process that leads to gross hematuria originating from the urinary bladder mucosa. A previously healthy 2.5 -year-old boy referred to our center $5 \mathrm{~h}$ after ingestion of $120 \mathrm{ml}(6 \mathrm{~g})$ of cephalexin suspension was presented with abdominal pain, diarrhea, vomiting, and gross hematuria. The results of a general physical examination were unremarkable except mild suprapubic tenderness. He was admitted to hospital and hydrated. Laboratory tests on admission showed a normal complete blood count (CBC) and electrolytes, normal PT and PTT, negative Coombs test, many RBCs per high-power 
field in urine, and normal urinary tract sonography. Within $24 \mathrm{~h}$, the urine cleared, showing only one to two red blood cells per high-power field and no changes in CBC, electrolytes, or kidney function tests. His urine culture was negative. Although there are reports of antibiotics-induced HC, the rarity of cephalosporine- and cephalexin-induced hemorrhagic cystitis encourage us to report this child.

(44) Decreased Level of Consciousness Due to Albizia julibrissin Poisoning: a Case Series

Khosrojerdi H, Amini M. Mashhad University of Medical Sciences, Mashhad, Iran

Introduction: Albizia julibrissin is a species of legume in the genus Albizia, native to southwestern and eastern Asia, from Azerbaijan to China and Korea. Albizia julibrissin overdose can cause seizure, tremor, staggering gait, convulsions, and labored breathing.

Methods: This is an observational study on three children (3, 3.5, and 5 years old) referred with Albizia julibrissin ingestion to our Medical Toxicology Resarch Centre in November 2010. All patients admitted with decreased level of consciousness. We checked serum levels of blood sugar, blood urea nitrogen, creatinine, $\mathrm{Na}, \mathrm{K}, \mathrm{CPK}$, and also CBC. We monitored the patients for cardiac arrythmia and any abnormality on ECG. Concurrent intoxication with central nervous system depressants was excluded.

Results: Decreased level of consciousness occurred after $20 \mathrm{~min}$ of ingestion. Coma lasted differently $(6,7$, and $7.5 \mathrm{~h}$ ). Return of consciousness occurred after this duration without any short-term neurologic sequella. Pupils' size and electrocardiography of admission time along with cardiac monitoring and blood glucose were normal.

Conclusion: Accidental ingestion of Albizia julibrissin by unknown mechanism can cause coma in children.

\section{(45) ECG Changes in Acute Tricyclic Antidepressant Poisoning in Children in Tehran 2007-2008}

Mehregan F.F., Farnaghi F, Kefayat M, Biranvand M.R., Gachkar L.

Pediatric Department, Loghman Hospital, Shaheed Beheshti University, Tehran, Iran

Objective: Intoxication with tricyclic antidepressant (TCA) is very important in concern of its nature and toxicity, especially cardiovascular and neurological complications. Researches mention the role of QRS $>100 \mathrm{~ms}$ and $\mathrm{R} / \mathrm{S}>0.7$ in ECG in predicting seizure and ventricular arrhythmia in TCA intoxication. We have done this study to evaluate ECG changes in TCA intoxication in pediatric patients.
Methods: This is a cross-sectional study on children with TCA intoxication who were referred to Loghman Hakim Hospital (a referral children poisoning center in Tehran) between August 2007 and August 2008. We took an electrocardiogram for all patients after TCA overdose. QRS width, the amplitude of the $\mathrm{R}$ waves, $\mathrm{S}$ waves, and $\mathrm{R} / \mathrm{S}$ ratio in AVR lead were measured on the initial ECG.

Results: We studied 36 children (17 boys, 19 girls) with TCA intoxication. Mean age was $5.5+4.9$ years (range, $1-$ 18 years). Eighty-one percent had sinus tachycardia. There was no seizure or ventricular arrhythmia. Thirty-nine percent of cases had QRS $>100 \mathrm{~ms}$ and $11 \%$ of patients had $\mathrm{R}>3 \mathrm{~mm}$ in AVR lead. None of them had R/S>0.7.

Conclusion: There was no seizure or ventricular arrhythmia among the patients in our study.

(46) Effect of Naloxane Trial in Decreased Level of Consciousness Due to Valproic Acid Poisoning: a Case Series

Khosrojerdi H, Amini M.

Mashhad University of Medical Sciences, Mashhad, Iran

Introduction: Valproic acid can cause convulsion, hyperammonia, altered level of conciousness, and arrythmia. Its mechanism may be through blokage of sodium and/or calcium channel. One study tried naloxone in this poisoning with the possible mechanism of opioid receptors. This study investigated the influence of naloxone trial on altered level of consciousness in valproic acid poisoning.

Methods: This is a prospective observational study on 18 valproic acid-overdosed patients admitted from September 2010 to June 2011. Eleven patients used it alone and four patients were admitted with coma. Physical examination revealed normal pupil sizes. We used naloxone in four comatous patients in escalating doses of $0.4 \mathrm{mg}$ intravenous naloxone, till response in counsiousness appeared (total of $1.6,2.4,2$, and $3.2 \mathrm{mg}$ in four patients). Urinary immunoassay of methadone, morphine, tramadol, LSD, phenobarbital, imipramine, amitriptyline, salisylate, phenothiazine, amphetamin, methamphetamine, phencyclidine, and cocaine were negative. There was no evidence of renal or hepatic failure.

Results: Mean $( \pm \mathrm{SD})$ age was $26.5( \pm 7.8)$ years. Other complaints of poisoned pateints included altered level of conciousness, abdominal pain, nausea, convulsion, vertigo, and headache. Dramatic return of consiousness after IV naloxone trial can be due to $\mu$-receptor blockade by this drug.

Conclusion: Naloxone by unknown mechanism can return coma in valproic acid overdose. Mechanism may be via agonism of both $\mu$-opioid receptor and potential $\mathrm{Ca}$ channel blockage. 
(47) Fatal Amlodipine Overdose: Challenges with Metformin and Alcohol Co-ingestion

Aaron Mark R. Hernandez ${ }^{1}$, Cherie Grace Quingking ${ }^{2}$, Lynn Crisanta Panganiban ${ }^{2,3}$, Adrian Paul Rabe ${ }^{4}$.

${ }^{1}$ Department of Medicine, University of the Philippines, Philippine General Hospital, Manila, Philippines.

${ }^{2}$ National Poison Control and Management Centre, Philippine General Hospital, Manila, Philippines.

${ }^{3}$ Department of Pharmacology, College of Medicine, University of Philippines, Manila, Philippines.

${ }^{4}$ Ateneo School of Medicine and Public Health, The Medical City, Pasig City, Philippines

Abstract Text:

A rare case of serious calcium channel blocker amlodipine with concomitant intake of metformin and alcohol is described and illustrated. The objectives are to describe the clinical features and course of amlodipine overdose and discuss the pitfalls and management strategies in the treatment of amlodipine overdose. Strategies in dealing with co-ingestion of metformin, an insulin sensitizer, which may cause alterations in the management of calcium channel blocker overdose are described.

Non-accidental overdose with $100 \mathrm{mg}$ of amlodipine of unknown brand caused generalized body weakness and severe hypotension in a 26-year-old male patient. He was initially managed with fluid resuscitation, and later dopamine and norephinephrine, which temporarily improved the patient's blood pressure. Activated charcoal, sodium sulfate and calcium gluconate were administered as decontamination and antidotal therapy accordingly. Insulin was not initiated at first due to the metformin co-ingestion. Persistent hypotension was controlled by inotrope titration. During ward admission, sudden onset of dyspnea was noted with signs of acute left ventricular dysfunction. After resuscitation, the patient eventually expired from fatal arrhythmia.

\section{(48) Fracture and Dislocation of Shoulder Due to Tramadol-Related Seizure: a Case Report}

Seyed Reza Mousavi, Seyed Khosro Ghasempouri, Azam Mihandost.

Medical Toxicology Research Center, Imam Reza Hospital, Faculty of Medicine, Mashhad University of Medical Sciences, Mashhad, Iran

Introduction: Tramadol is a synthetic analgesic with both opioid and nonopioid mechanisms responsible for its clinical effects. A large number of spontaneous reports to the Food and Drug Administration suggest that therapeutic use of tramadol may cause seizure. Tramadol-related seizures are suppressed with benzodiazepines. Acute overdose of tramadol is generally considered non-life threatening, and most fatalities were associated with polysubstance overdose.

Materials and Methods: This study is a case report that represents fracture and dislocation of shoulder due to tramadol-related seizure in a 21-year-old man that had consumed a $600 \mathrm{mg}$ single dose of tramadol.

Results: The patient has been irregularly consuming $100 \mathrm{mg}$ of tramadol, almost weekly in the past 2 years. He has not any history of previous seizure or spontaneous dislocation of the shoulder. The seizure was revealed less than $1 \mathrm{~h}$ after consumption. The left shoulder radiography demonstrated dislocation of the head of humerus with fracture of its greater tuberosity.

Conclusion: Although seizure is a rare complication of therapeutic dose of tramadol, it can be associated with serious complications.

\section{(49) Intravenous Fat Emulsion, a Promising Novel} Universal Antidote

Maryam Vahabzadeh, Mahdi Balali-Mood.

Medical Toxicology Research Center

Introduction: Intravenous fat emulsions (IFE) have been extensively utilised for many decades as a source of essential fatty acids and calories in critically ill patients. In the 1990s, therapeutic effects of IFE on the cardiovascular and central nervous system disorders caused by local anaesthetics and other acute poisonings in experimental models were reported. Many investigations have been performed since then and its clinical applications in some human acute poisonings were promising.

Mechanism of Therapeutic Effects: The main mechanism suggested for antidotal effect of IFE is called 'lipid sink formation', by which repartitioning of the drug occurs within the blood and away from the site of toxicity. Alternative mechanisms of action are improvement of ATP synthesis by increasing the intracellular fatty acid in the cardiac cells and positive inotropic effect by rising calcium amounts within the myocyte.

Reported Studies: Systematic search of the medical literature revealed that by July 2011, 50 human cases and 22 animal studies of IFE administration in lipophilic drugs overdose have been reported. Available data gathered from experimental studies and case reports imply a prospective advantage of using IFE in potentially life-threatening acute poisonings due to drugs such as tricyclic antidepressants, haloperidol, ropivacaine, bupivacaine, verapamil, mepivacaine, a number of beta-blockers and calcium channel blockers.

Conclusion: More experimental investigations and clinical trials on the therapeutic effects of IFE in acute pharmaceutical and chemical poisonings are required to confirm its safety and efficacy as a universal antidote. 


\section{(50) Pattern of Addiction in Hospitalized Male Patients}

Abbas Aghabiklooei*, Babak Mostafazadeh, Nasim Zarrin. Clinical Toxicology Service, Firouzgar Teaching Hospital, Tehran University of Medical Sciences, Tehran, Iran

Background: Opioid addiction remains a main medical and social problem in most of the countries. Also, opium or heroin overdose or withdrawal syndrome is one of the most common medical problems among hospitalised addicted patients and this can complicated the patients' medical status. Although, recent researches have shown that the substance pattern has switched to illicit drugs, amphetamine and its derivates. This article aimed to evaluate the pattern of the uses of these substances among male patients who were admitted in a general hospital on 2008-2009.

Methods: This is a cross-sectional article. Data were obtained from all patients who had history of substance usage and who admitted to different wards of Firouzgar Teaching Hospital from 2008 to 2009 in Tehran, Iran.

Results: Of the total 2,782 male admitted patients, $29.9 \%$ (832 cases) gave history of one or more usage of opium or illicit drug in the past 2 weeks. Six hundred eleven patients $(21.96 \%)$ were chronic substance abuser with mean age of 46.2 years ( $\mathrm{min}$, 16 years; max, 78 years). Of these, $79.1 \%$ were opium users followed by amphetamine (12.9\%) and heroin (5.2\%). Seventy percent were users of narcotics combined with CNS stimulants (amphetamines or cannabis) at the same time. Smoking was the most common way and was found in $83.2 \%$.

Conclusion: Opium had the first state among other substances but the pattern tends to change into amphetamine and new substances, especially in Iranian juvenile.

\section{(51) Rhabdomyolysis in Patients with Acute Poisoning}

Seyed Reza Mousavi, Mahdi Balali-Mood, Reza Afshari, Mansooreh Vafaee, Masoumeh Hoseini.

Medical Toxicology Research Center, Imam Reza Hospital, Faculty of Medicine, Mashhad University of Medical Sciences, Mashhad, Iran

Introduction: Rhabdomyolysis is a syndrome caused by injury to skeletal muscle and with leakage of potentially toxic cellular contents (myoglobin and potassium) into the systemic circulation. Myoglobinuria is reported to cause acute renal failure (ARF) in 15-33\% of cases. Drugs and alcohol have become frequent causative agents in up to $81 \%$ of cases with rhabdomyolysis. In this study, we aimed to investigate rhabdomyolysis in a large population of hospitalized patients with acute poisonings.

Materials and Methods: This study was a prospective cross-sectional study. For 1 year (April 2009 to March 2010) from 12,260 patients who were referred to Imam Reza
Hospital, Toxicology Center, 3,555 were admitted to the Toxicology Ward. The diagnosis of rhabdomyolysis was defined as the serum creatine kinase value was five times greater than the upper limit of the normal level (195 IU/L). ARF was defined as an increase in serum creatinine above $2 \mathrm{mg} / \mathrm{dl}$ that had no response to initial hydration.

Results: From 3,555 admitted patients, 126 cases revealed rhabdomyolysis. Of these, 91 and 35 were males and females, respectively. Their ages varied between 15 and 85 $(32.5 \pm 14)$ years. Most patients were in the age group of 20 30 years old. The most common acute poisoning associated with rhabdomyolysis included: opioid (26\%), alcohol (7\%), and monoxide carbon (7\%). ARF was present in $18(14 \%)$ patients. Among these, two patients with ARF needed to hemodialysis and six patients died.

Conclusion: Rhabdomyolysis was found as a usual complication in acute poisoning. The most common causative poisons were opioid, alcohol, and monoxide carbon. ARF was the most serious complication of rhabdomyolysis.

\section{(52) Serotonin Syndrome Following Single Ingestion of High-Dose Amphetamine}

Seyed Hesam Rahmani ${ }^{1}$, Sajjad Ahmadi ${ }^{1}$, Samad Shams Vahdati ${ }^{1}$, Hossein Hasssanian Moghaddam ${ }^{2}$.

${ }^{1}$ Tabriz University of medical sciences, Tabriz, Iran.

${ }^{2}$ ShahidBeheshtiUniversity of Medical Sciences, Tehran, Iran

Serotonin syndrome is a potentially life-threatening adverse drug reaction that may occur following therapeutic drug use, inadvertent interactions between drugs, overdose of particular drugs, or the recreational use of certain drugs. It is most commonly reported with combinations of serotonergic medications. Serotonin syndrome is not an idiosyncratic drug reaction. Amphetamine is a psycho-stimulant drug of the phenethylamine class which produces increased wakefulness and focus in association with decreased fatigue and appetite. An amphetamine overdose is rarely fatal but can lead to a number of different symptoms, including psychosis, chest pain, and hypertension. It is among the agents with moderate potency to produce serotonin syndrome. Here, we present the first reported case of serotonin syndrome after a single ingestion of high-dose amphetamine (body stuffer of $2 \mathrm{~g}$ amphetamine) in 28-year-old previously healthy man without any drug history, $2 \mathrm{~h}$ prior to admission. Clinical manifestation included hyperthermia, hypertension, increased heart rate, sweating, dilated pupil, confusion, overactive reflexes and clonus that are greater in the lower limbs than in the upper limbs. A complete blood count was normal except for severely elevated white blood cell count of $22.3 \times 103 / \mu \mathrm{L}(22.3 \times 109 / \mathrm{L})$ with $74 \%$ neutrophils and $24 \%$ lymphocytes. His serum blood urea nitrogen, creatinine, liver function tests, and electrolytes were normal. Mild elevation in 
serum creatinine kinase was seen. Coagulation studies and urine analysis were normal. Serum glucose was $210 \mathrm{mg} / \mathrm{dL}$; arterial blood gases show metabolic acidosis. We intubated him by rapid sequence intubation method and then took an emergency abdominal CT scan for any need for surgical intervention; we admitted him in the ICU Ward and did supportive care including the control of agitation, autonomic instability, and hyperthermia. After 2 days, we extubated him and after 5 days he was discharged from hospital.

(53) The Rise of Aminotransferase Following Hepatotoxicity from Paracetamol Overdose

Gregory Cham, Chan Wui-Ling, Lin Miao-Xin.

Emergency Department, Tan Tock Seng Hospital, Novena, Singapore

Context: We studied the rate of aminotransferase elevation from hepatotoxicity due to paracetamol overdose.

Methodology: A retrospective review of case records was performed, consisting of patients who presented from 1 Jan 2006 to 31 August 2009 for paracetamol overdose and treated with IV $N$-acetylcysteine. Patient data was accrued from computerized and hard-copy clinical data, laboratory database of all paracetamol assays and administrative data of patients with a discharge diagnosis of paracetamol overdose. Patients with incomplete data from unknown toxicity, unknown treatment, and those who left before $24 \mathrm{~h}$ against medical advice were excluded. Hepatotoxicity was defined as alanine aminotransferase (ALT) or aspartate aminotransferase (AST) levels of $1,000 \mathrm{U} / \mathrm{L}$ or higher.

Results: Among 188 patients in the retrospective cohort, 8 developed hepatotoxicity. ALT began to rise at $14.5 \mathrm{~h}$ after overdose at an overall rate of $0.67 t^{1.87}\left(R^{2} 0.68\right),(t$ is the time in hours after overdose). AST begins to rise after $9 \mathrm{~h}$ post-ingestion at the rate of $0.73 t^{1.81}\left(R^{2} 0.51\right)$. Significant elevation of greater than $100 \mathrm{U} / \mathrm{L}$ was delayed by as much as $27 \mathrm{~h}$ for ALT and $32 \mathrm{~h}$ for AST on some patients.

Conclusion: Aminotransferase levels begin to rise early and exponentially when hepatotoxicity sets in, but significant elevations in the early stages may be delayed in some patients.

\section{(54) Tramadol Overdose Induces a Short-Lived U-Wave in Pre-cordial Leads}

Khosrojerdi H, Afshari R,Shojaie R.

Medical Toxicology Research Center, Mashhad University of Medical Science, Mashhad, Iran

Introduction: Tramadol overdoes is common in Iran. Electrocardiographic changes related to this overdose have been reported to be minor. This study aimed at evaluating the presence of $U$ waves in admitted tramadol overdose.
Methods: All admitted cases in the Medical Toxicology Centre, Imam Reza (p) University Hospital with tramadol overdose were prospectively studied from 20 September 2010 to 20 June 2011. All cases with hypomagnesaemia and hypocalcaemia were excluded. Subjects with positive screened tests for other drugs were excluded. ECG was taken on admission, 6 and $12 \mathrm{~h}$ after admission.

Results: Seventy-one cases were studied in total. Mean (SD, min-max) age was $23.6(4.2,18-33)$ with a male percentage of $66 \%$. On admission, systolic blood pressure was $113(15$, 90-140), diastolic blood pressure was $74(8,60-90)$. Heart rate was $100(10,80-122)$. Presence of $U$ wave is summarized in the table.

\begin{tabular}{llllllll}
\hline Leads & Admission & $+6 \mathrm{~h}$ & $+12 \mathrm{~h}$ & Leads & Admission & $+6 \mathrm{~h}$ & $+12 \mathrm{~h}$ \\
$\mathrm{DI}$ & 2.8 & 2.8 & 0.0 & $\mathrm{~V}_{1}$ & 18.3 & 8.5 & 0 \\
$\mathrm{D} 2$ & 1.4 & 1.4 & 0.0 & $\mathrm{~V}_{2}$ & 50.7 & 21.1 & 0.0 \\
$\mathrm{D} 3$ & 1.4 & 0.0 & 0.0 & $\mathrm{~V}_{3}$ & 64.8 & 26.8 & 0.0 \\
$\mathrm{aV}_{\mathrm{R}}$ & 1.4 & 0.0 & 1.4 & $\mathrm{~V}_{4}$ & 70.4 & 28.2 & 1.4 \\
$\mathrm{aV}_{\mathrm{L}}$ & 1.4 & 1.4 & 0.0 & $\mathrm{~V}_{5}$ & 60.6 & 26.8 & 1.4 \\
$\mathrm{aV}_{\mathrm{F}}$ & 1.4 & 0.0 & 0.0 & $\mathrm{~V}_{6}$ & 38.0 & 6.9 & 1.7 \\
\hline
\end{tabular}

As can be seen, while $\mathrm{U}$ waves are rare in limb leads, more than $50 \%$ of pre-cordial leads, in particular $\mathrm{V}_{2}$ to $\mathrm{V}_{5}$, were observed to express $\mathrm{U}$ waves. This phenomenon reduced to half after $6 \mathrm{~h}$ and almost disappeared in $12 \mathrm{~h}$.

Conclusion: This study expands previous findings in which changes in ECG indices were reported to be limited to presence of a short-lived $U$ wave in pre-cordial leads, which is independent from electrolyte imbalances.

\section{(55) Tramadol Overdose Induces Paresthesia and Hypotonia in Acute Phase of Poisoning}

Khosrojerdi H, Afshari R, Ghasempoori K.

Medical Toxicology Research Center, Mashhad University of Medical Science, Mashhad, Iran

Introduction: Tramadol overdose is relatively common in Iran. While seizure, limited consciousness were reported due to tramadol overdose, no reports exist in regard to hypotonia and paresthesia related to this overdose.

Methods: All admitted cases in medical toxicology centre, Imam Reza (p) University Hospital with tramadol overdose were prospectively studies from 20 September 2010 to 20 June 2011. All cases with previous use of other medications or history of seizure were excluded. Neurologic physical exam were performed on admission, 6 and $12 \mathrm{~h}$ after admission. Urinary tramadol was positive for all cases.

Results: One hundred two cases were studied in total. Presence of paresthesia and mean (SD, min-max) force are summarized in the table. Deep tendon reflexes were 
increased in $17.6 \%$ on admission. It was disappeared after $6 \mathrm{~h}$. Tones were in normal ranges. As can be seen, paresthesia in lower and upper limbs happen in more than $50 \%$ of cases on admission, which was more common in the lower limbs $(P<0.001)$. Paresthesia in the lower, but not in the upper limbs, was observed up to $6 \mathrm{~h}$. Similar pattern observed with lower forced on admission. Muscle force in lower and upper were significantly lower on admission, in comparison to $6 \mathrm{~h}$ later.

\begin{tabular}{llllllll}
\hline Paresthesia & Admission & $+6 \mathrm{~h}$ & $+12 \mathrm{~h}$ & Force & Admission & $+6 \mathrm{~h}$ & $+12 \mathrm{~h}$ \\
Lower limbs & 63.7 & 25.5 & 1.0 & Lower limbs & $3.5(1.0,2-5)$ & $4.6(0.5,4-5)$ & $5.0(0.0,5-5)$ \\
Upper limbs & 50.0 & 4.9 & 0.0 & Upper limbs & $4.24(0.6,4)$ & $5.0(0.2,4-5)$ & $5.0(0.0,5-5)$ \\
\hline
\end{tabular}

Conclusion: This study expands our clinical judgment that tramadol can induce transient hypotonia and paresthesia which is independent from electrolyte imbalances.

\section{(56) Tramadol-Induced Seizures and Trauma}

Hossein Hassanian-Moghaddam ${ }^{1,2}$, Hoorvash Farajidana ${ }^{1,2}$, Nasim Zamani ${ }^{1,2}$, Hossein Sanaei-Zadeh ${ }^{1,2}$.

${ }^{1}$ Department of Forensic Medicine and Toxicology, Tehran University of Medical Sciences, Hazrat. Rasoul Akram Hospital, Tehran, Iran.

${ }^{2}$ Department of Clinical Toxicology, Loghman-Hakim Poison Hospital, Shahid Beheshti University of Medical Sciences, Tehran, Iran.

Background and Objectives: The objective of this study is to determine the prevalence, type and site of the injuries due to tramadol-induced seizures in the patients who had referred after its overdose or use of its therapeutic dose.

Materials and Methods: All patients referring to Loghman Hakim Poison Hospital (February 2009 to April 2010) due to tramadol-induced seizures were included. The patients' data including age, gender, ingested dose by history, route of exposure, manner of poisoning, previous history of suicidal attempts, previous history of drug or substance abuse, history of tramadol abuse, type of the seizure, number of episodes of seizure before presentation or after admission, site of the trauma, and type of injury were recorded. Statistical analysis was done using SPSS software.

Results: A total of 232 patients were included in the study. Of them, 185 (79.7\%) had referred within the first $6 \mathrm{~h}$ after ingestion. The mean dose ingested by history was $1,416 \pm$ $1,124 \mathrm{mg}$. History of tramadol abuse was positive in 114 (49.1\%) of the patients. Seizure episodes had occurred once in $207(89.2 \%)$, twice in $21(9.1 \%)$, and three times in 4 $(1.7 \%)$ of the patients. The prevalence of trauma was $24.6 \%$ (in 57 patients) with the most frequent site of trauma to be the face $(9.5 \%)$ followed by shoulder $(4.3 \%)$, head (3\%), trunk (1.7\%), and upper extremities $(1.3 \%)$. No statistically significant difference was found between patients with and without trauma associated with tramadolinduced seizure in terms of age, gender, ingested dose by history, positive history of addiction to other opioids, and number of episodes of seizure.

Conclusions: The only serious injury associated with this type of seizure is head injury which is present in approximately $1 \%$ of the patients.

\section{(57) A Case of Suicide by Formic Acid Ingestion}

Abul Fazil ${ }^{1,2}$, Ramdas Naik ${ }^{1,2}$, Ritesh G. Menezes ${ }^{1,2}$.

${ }^{1}$ Kasturba Medical College (Affiliated to Manipal University), Mangalore, India.

${ }^{2}$ Srinivas Institute of Medical Sciences and Research Centre, Mangalore, India

Formic acid is commonly used in the rubber industry. It is used to make rubber sheets from latex. Eighty-five percent is the usual strength of formic acid available in the Indian market. The role of formic acid is to change the consistency of latex. Latex is milk like. If its water content is reduced without adding formic acid, it forms sponges and foams. If formic acid is added, it gets thick. This is pressed between rollers in the rubber industry and made into sheets. Thus formic acid is easily available in the regions of rubber plantation and rubber industry in India. We herein present a fatal case of suicidal ingestion of formic acid in an adult young male. Following ingestion of the corrosive, the victim had survived for an estimated period of less than $18 \mathrm{~h}$. Postmortem external examination of the body revealed corrosive chemical burns on the lower half of the face on the left side. Internal examination revealed grayish-black-coloured corrosive chemical burns of the oesophageal mucosa, gastric mucosa and the proximal small intestinal mucosa. Blackish discolouration of the rectus sheath, omentum, external surface of the liver and spleen was observed. Histopathology revealed dilated and congested blood vessels in the oesohageal subepithelium, complete sloughing of the gastric mucosa, extensive ulceration of the small intestinal mucosa and omental fat necrosis. 


\section{(58) Buprenorphine-Induced Withdrawal Symptoms in a Methadone-Dependent Subject}

Rowshanak Afshar, Seyyed Amir Askari, Reza Afshari. Medical Toxicology Centre, Mashhad, Iran

Introduction: Buprenorphine is a partial opiate agonist that has been registered in Iran for the management of opiate withdrawal and as a substitution to methadone in case of maintenance therapy. Since buprenorphine is a partial agonist, administration of this medication to naive cases may lead to agonist activities. In contrast, it is plausible that this medication could precipitate withdrawal symptoms in a dependent subject.

Case Report: A 61-year-old- man referred to the toxicology triage due to severe withdrawal symptoms. In his past medical history, he was on methadone maintenance therapy for the treatment of opiate addiction for 3 months. He was given $4 \mathrm{mg}$ sublingual buprenorphine as a part of a new protocol of treatment. After half an hour, he experienced agitation, cold sweat, tremor in his extremities, weakness and pain in the whole body. He admitted for the severity of the symptoms and received fluids and benzodiazepines and clonidine. He was discharged after $24 \mathrm{~h}$ when the symptoms declined.

Discussion: This case demonstrates the difficulties in changing methadone to buprenorphine which could lead to precipitation of withdrawal symptoms. It could be recommended that patients on methadone therapy should experience a longer gap before administration of buprenorphine and if withdrawal symptoms happen, observation, fluids and benzodiazepines are probably enough.

\section{(59) Case Report: Copper Sulfate Poisoning From Thai Traditional Medicine}

Kitisak Sanprasert, Satariya Trakulsricha, Achara Tongpo, Charuwan Sriapha, Sunun Wongvisawakorn, Panee Rittilert, Sming Kaojarern, Winai Wananukul.

Ramthibodi Poison Center, Faculty of Medicine, Ramathibodi Hospital, Mahidol University, Bangkok, Thailand

Introduction: Copper sulfate is mainly used in industry. Some use as an ingredient in Thai traditional medicine. Copper sulfate poisoning is rare. This paper reports three cases of copper sulfate poisoning from Thai traditional medicine used to treat alcohol dependence in Thailand.

Case Report: Three patients drank Thai traditional medicine recipe for treating alcohol addicts. It was obtained from a temple. Five days after having taken Thai traditional medicine, they developed severe diarrhea, vomiting, upper gastrointestinal bleeding, hepatitis, hemolytic anemia, methemoglobinemia accompanied by acute renal failure. All patients were treated with dialysis and supportive care. No one received chelating agents and one of them died. Sepsis was the cause of death in this patient. The sample of Thai traditional medicine was found to contain a significant amount of copper sulfate (19,450 ppm).

Conclusion: Copper sulfate is a highly toxic agent but sometimes used as an ingredient in herbal medicine. Poisoning can cause severe local and systemic toxicity. The mainstay treatment is supportive treatment.

(60) Cytotoxic and Apoptogenic Properties of Sophora pachycarpa and Salvia chorassanica in Human Cancer Cell Lines

Seyed Hadi Mousavi, Mahsa Hossini Motaez, Hojat Soufi, Seyed Ahmad Emami, Zahra Tayarani-Najaran.

Pharmacological Research Centre of Medicinal Plants, School of Medicine, Mashhad University of Medical Sciences, Mashhad, Iran.

Sophora pachycarpa C.A. Mey. is a plant from the family of Fabaceae. Some species of the genus Sophora had shown to induce anti-proliferative effect as well as apoptosis in cancer cells. However, there is no available information to address about $S$. pachycarpa. Here, we investigated the cytotoxic effects of methanol extract and obtained fractions from $S$. pachycarpa and Salvia chorassanica root extract on different cancer cell lines including A549, HeLa, HL60, MCF-7, and PC3 cells, and lymphocytes as non-malignant cells. Meanwhile, the role of apoptosis was explored in this toxicity. Malignant and non-malignant cells were cultured in RPMI 1640 medium and incubated with different concentrations of plant extracts. Cell viability was quantified by MTT assay. Apoptotic cells were determined using PI staining of DNA fragmentation by flow cytometry (sub-G1 peak) and activity of caspase- 3 , caspase- 8 and caspase- 9 . The degree of DNA fragmentation was analyzed using agarose gel electrophoresis based on the formation of internucleosomal units. S. pachycarpa inhibited the growth of malignant cells in a dose-dependent manner and the $\mathrm{CH}_{2} \mathrm{Cl}_{2}$ fraction showed the lowest $\mathrm{IC}_{50}$ values ranged from 30 to $50 \mu \mathrm{g} / \mathrm{mL}$ in various cell lines. The IC50 for methanol extract, $n$ hexane, CH2CL2 fractions for $S$. chorassanica were calculated $8.8,5.45$, and $2.38 \mu \mathrm{g} / \mathrm{ml}$ in HeLa cells. S. pachycarpa and $S$. chorassanica induced a sub-G1 peak in flow cytometry histogram of treated cells, DNA fragmentation and activation of caspase- 3 and caspase- 8 acivity indicating apoptotic cell death is involved in $S$. pachycarpa and $S$. chorassanica-induced toxicity. In conclusion, S. pachycarpa and S. chorassanica exert cytotoxic effects in different cancer cell lines in which apoptosis plays an important role. IC50 values show both plants could be considered as a potential and promising chemotherapeutic agent in cancer treatment. 
(61) Delayed Cutaneous Manifestations of Sulfur Mustard Gas Poisoning in Iranian Veterans North East: 2227 Years After Exposure

Pouran Layegh, Masood maleki, Mahdi Balali mood, Seyed Reza Mousavi, Hadis Yousefzadeh.

Research Center for Skin Diseases and Cutaneous leishmaniasis, Mashhad University of Medical Sciences Research center of Clinical Toxicology, Mashhad University of Medical Sciences

Background: Sulfur mustard (SM) poisonings induces acute and delayed skin manifestations. We aimed to evaluate delayed cutaneous manifestations of SM poisoning in Iranian veterans and comparing its results with acute phase.

Methods: Exact clinical examinations of skin and mucous membrane were performed on 43 SM-poisoned Iranian Veterans who had $40 \%$ or more disability rate according to directions of Veterans Foundation in Khorasan, North East Iran.

Results: Forty-three male patients, 40 to 71 years old whose SM poisoning dated back to 22-27 years ago, were evaluated. The most common skin manifestation in acute phase was recorded as erythema with superimposed blister $(25.6 \%)$, blister (23.3\%), and erythema with hyperpigmentation (14\%). The commonest delayed dermatological complaints were itching $(23.3 \%)$ and combination of dryness, burning, and dysesthesia (76.7 \%). Most complaints were restricted to skin folds (74\%), external genitalia (51.2 \%), face and neck (48.8\%), upper limb (39.5\%), and lower limb (48.8\%). Although $41.9 \%$ of these complaints were compatible with the first site of SM acute poisoning, there was not any statistical difference between them. There is significantly considerable correlation between the late dermatological complaint in skin folds and acute ones $(P=0.014)$. The most significant findings of physical examination were normal skin color (72.1\%), hyperhidrosis (67.4\%), dryness (44.2\%), poikiloderma (16.3\%), and hypohidrosis (11.6\%). The most common clinical diagnosis was multiple cherry angioma (72.1 \%), seborrheic keratosis (28\%), multiple junctional nevus (18.6\%), echymoses (18.6\%), poikiloderma (16.3\%), dryness and burning (11.6\%).

Conclusion: Certain regions of the body, such as genitalia and axilla, are more vulnerable to SM since these sensitive areas are generally thinner and have more follicles which naturally facilitate SM penetration. Various late cutaneous complication including hyper- and hypo-pigmentation, eczema, atrophy, and dry skin have already been shown to have an association with SM exposure that are compatible with our results.
(62) Demographic Pattern and Severity of Caustic Injury in Caustic Ingestion Patients Admitted in Loghman Medical Center

Hajinasrollah ESMAEIL MD., Salehi Nourallah MD., Khoshkar Ali MD, Peyvandi Hassan MD.

Departemant of Surgery, Loghman Medical Center, Shahid Behshti Medical University, Tehran, Iran

Objective: The objective of this study is to report frequency and ratio of caustic substance ingestion cases and their demographic data when admitted in Loghman Medical Center, as a referral center in toxicological problems.

Cases and Methods: From 2007 to 2010, 300 cases of caustic ingestion were admitted. One hundred and eighty nine men and 109 women ingested caustic ingestion. All demographic data, past medical and past psychiatric history were recorded. Gastroesopagheal endoscopy was done. Caustic injury was defined based on Zargar grading system. Data were analyzed with SPSS 18 software, accordingly.

Results: Three hundred cases with corrosive substance ingestion were admitted in Loghman Medical Center during 2007 to 2010 . One hundred fifty-four patients $(51.3 \%)$ were single, 83 cases $(27.6 \%)$ were married, 34 cases $(11.2 \%)$ were divorced, and 39 cases $(13 \%)$ were widowed. Ingestion was associated with suicidal intent in 217 cases (72.3\%). One hundred thirty-four cases (44.6\%) had a previous history of psychiatric issues; 98 cases $(32.6 \%)$ had history of substance abuse; mean time interval was $8.45 \pm 2.11 \mathrm{~h}$. Amount of caustic substance was between 1 cup ( $75 \mathrm{cc})$ and 12 cups $(900 \mathrm{cc})$. The caustic materials for injuries were: in 97 cases $(32.4 \%)$, chemical depilatory agent (Vajebi); 73 cases $(24.3 \%)$, chemical laundry liquids; 54 cases (18\%), liquid lye; and 25 cases (8.3\%), acids. Based on Zargar system 128 cases did not have esophageal and gastric burn, 116 cases had grade I injury, 38cases had grade II injury, and 14 cases had grade III injury.

Conclusion: Caustic ingestion is a suicidal event in Middle East and Asia. Singles, men, and cases with psychiatric problems are more prone to suicide, and thus to caustic ingestion.

\section{(63) Diversin from Ferula diversivittata Protects Human Lymphocytes Against Oxidative Stress Induced by $\mathrm{H}_{2} \mathrm{O}_{2}$}

Ramin Rezaee, Hassan Zarei, Effat Behravan, Fatemeh Soltani, Fatemeh Mosaffa, Mehrdad Iranshahi, Javad Behravan

Oxidative stress caused by reactive oxygen species has been shown to contribute to aging and apoptosis process, thus finding new antioxidant agents especially naturally occurring ones is of great interest. In this study, we evaluated the 
antigenotoxic effects of diversin, a prenylated coumarin obtained from Ferula diversivittata roots using Comet assay. Lymphocytes were isolated from blood samples taken from healthy volunteers and incubated with diversin $(10,25$, $50,100,200$, and $400 \mu \mathrm{M}$ ) alone, or in the presence of $\mathrm{H}_{2} \mathrm{O}_{2}$ $(25 \mu \mathrm{M})$. DNA breakage was measured based on the \%tail DNA and was compared with different concentrations of curcumin $(10,25$, and $50 \mu \mathrm{M})$ as the positive control. It was shown that all concentrations of diversin significantly reduce DNA damage caused by $\mathrm{H}_{2} \mathrm{O}_{2}$.

(64) Evaluation of Antigenotoxic Effect of 7Hydroxycoumarin and 7-Isopentenyloxy Coumarin on Human Peripheral Lymphocytes Exposed to Oxidative Stress

R. Rezaee, M. Iranshahi, J. Behravan, E. Behravan, F. Soltani.

Coumarins are a large class of natural derivatives mainly found in the families Umbelliferae and Rutaceae. DNA damage and oxidative stresses are accepted to be major factors in many degenerative diseases and in the aging process. Single-cell electrophoresis (also known as Comet assay) is a sensitive, rapid, and reliable method for evaluation of genotoxic effects. Protective properties of two prenylated coumarins: "7-isopentenyloxy coumarin" and "7hydroxy coumarin" were tested on human peripheral lymphocytes DNA lesions after exposure to $\mathrm{H}_{2} \mathrm{O}_{2}$-induced oxidative stress using the single-cell gel electrophoresis technique under alkaline condition. Lymphocytes were isolated from healthy volunteers' blood samples. The lymphocytes were incubated with 7-isopentenyloxy coumarin $(10$, $25,50,100$, and $200 \mu \mathrm{M}$ ) alone or with 7-isopentenyloxy coumarin $(10,25,50,100$, and $200 \mu \mathrm{M})$ in the presence of $\mathrm{H}_{2} \mathrm{O}_{2}(25 \mu \mathrm{M})$. The same procedure were done for 7 hydroxy coumarin $(10,25,50,100$, and $200 \mu \mathrm{M})$. In this study, ascorbic acid $(10,25,50,100$, and $200 \mu \mathrm{M})$ and $\mathrm{H}_{2} \mathrm{O}_{2}(25 \mu \mathrm{M})$ were considered as known antioxidant agent and positive control, respectively. Lymphocytes, which were treated by PBS only, were assumed as the negative control. The extent of DNA migration was analyzed with "TriTek Comet Score 1.5" software and the DNA damage was expressed as DNA tail percentage. As it was observed in this experiment, 7-isopentenyloxy coumarin and 7-hydroxy coumarin both exhibited significant antigenotoxic effect via decreasing DNA migration in comparison with non-treated group. It must be mentioned that antigenotoxic effect of 7hydroxy coumarin was greater than that of 7- isopentenyloxy coumarin. It is suggested that antigenotoxic properties of these substances is of great pharmacological importance and might be beneficial for cancer prevention.
(65) Evaluation the Ectotoxicological Effects of Nitrate in Lemna minor L.: a Tiny Aquatic Plant; Assessing the Phytoremediation Technology in the Nitrate Removal

Hadis Yousefzadeh ${ }^{1,2}$, Mohammad Sadegh Amiri ${ }^{1,2}$, Zahra Maleki ${ }^{1,2}$.

${ }^{1}$ Islamic Azad University, Mashhad Branch, Mashhad, Iran. ${ }^{2}$ Payam Noor University, Dargaz branch, Dargaz, Iran.

Contamination of drinking water by nitrate as the most ubiquitous chemical contaminant is increasing and an evolving public health challenge due to its potent carcinogenicity. The water plant Lemna minor is an angiosperm with a worldwide distribution. It is a free-floating plant, easy to culture in laboratory. This species takes up nutrients and all chemicals in the growth medium directly into the lower surface of its green fronds. It has been used as an experimental model system to investigate heavy metal-induced responses and to remove water pollutions. The basis of sampling method was non-randomization to assess the tolerance and effect of heavy metals pollution on the duckweed L. minor, the aquatic plants were exposed to different concentrations of nitrate $(0,30,60$ and $90 \mathrm{mg} / \mathrm{l})$. The samples (64) were taken below duckweed cover after every 2 days in seven steps to assess nitrate and chlorophyll concentrations. The amounts of $\mathrm{NO}_{3}$ detected in control duck weed were $21.33 \pm 5.05 \mathrm{ppm}$ that was ranged from 10.13 to $30.83 \mathrm{ppm}$ during experiments. While this amount ranged from 10.36 to $26.74 \mathrm{ppm}$ and 24.73 to $46.3 \mathrm{ppm}$ with increasing 30 - and 60 -fold $\mathrm{NO}_{3}$ exposure. The increasing uptake was more detected in $90 \mathrm{ppm} \mathrm{NO}_{3}$ concentration. The increasing for pigments (chlorophyll contents) were not seen for 30,60 and $90 \mathrm{ppm}$ in $\mathrm{t} 3$ and t7 days after treatment. And in these days, chlorophyll a and chlorophyll $\mathrm{b}$ had decreased in a non-significant order $(P=0.31)$. In the present study, biological absorption coefficient index increased according to increasing $\mathrm{NO}_{3}$. So the $\mathrm{NO}_{3}$ absorption by duck weed was found in 90, 60 and $30 \mathrm{ppm}$. Results show that duckweed tolerated nitrate at low concentrations without showing any significant changes in chlorophyll amount.

(66) Factors Related to Use of Rice Wine (Tapoy) Among Families in a Municipality in Cordillera Region, Philippines

Jerry Sagabaen Jr., Joselito Pascual, Erle Castillo, Ivanhoe Escartin.

${ }^{I}$ Postgraduate Institute of Medicine, College of Medicine, University of the Philippines, Manila, Philippines.

${ }^{2}$ Department of Health, Manila, Philippines.

${ }^{3}$ Cagayan Valley Medical Center, Carig, Tuguegarao City, Cagayan Valley, Philippines. 
Background: Drinking alcohol among Filipinos has been a way of life since ancient times and still is the most commonly abused substance up to the present. In the Philippines, commercial alcohol production has taken over smallscale alcohol production. Nevertheless, alcohol prepared at home continues to proliferate in the provinces such as distilled coconut spirit (lambanog), fermented coconut wine (tuba), and sugarcane wine (basi). In the Cordillera Region, rice is fermented to wine called tapoy and continues to be revered by community members as a traditional beverage commonly drunk by the Ifugaos not only during important ritual and feasts but as part of their way of life. As such, use, abuse, and dependence to this particular alcohol preparation are common.

Aim: This study describes related factors associated with rice wine use, abuse, and dependence.

Design and Settings: This study utilizes a cross-sectional study design.

Research Site: The study was done in the municipality of Alfonso Lista, Ifugao.

Research Subjects: One thousand two hundred forty-seven respondents representing 10-20\% of 10 barangays among 20 barangays of Alfonso Lista, Ifugao using stratified sampling method were included.

Results: Majority of the respondents were at a younger age group (14-27, $37.0 \%$ ), slightly dominated by male group $(52.9 \%)$, married $(62.3 \%)$, attained high school education (43.3\%), and unemployed (44.8\%). Among those employed, occupation category 5 (farmers, forestry workers, and fishermen) comprises $30.6 \%$ with an income bracket of P $1-10,000 /$ month (86.1\%). Majority of the respondents were of Ilocano tribe $(54.5 \%)$ and Ifugao tribe $(27.2 \%)$, belonging to the Roman Catholic group (54.6\%) and Pentecost group (16.2\%). Majority of those married had two to three children (45.8\%). Rice wine drinkers comprise 401 or $32.2 \%$ of the total respondents. Among the rice wine drinkers, those in the age group 28-41, male, married, belonging to occupational group 5, belonging to the Ifugao tribe, and member of the Evangelical group had more predilections for rice wine consumption. Rice wine drinkers who are 18 years old and above who started drinking at age below 18 comprises 148 of the total rice wine drinkers, and those who are below 18 years old comprises of 9 of the total rice wine drinkers. There is decreasing trend among newer generation towards rice wine consumption. However, it seems that other tribes had been so much influenced by the Ifugao tribe. Among rice wine drinkers, majority experienced acute effects in decreasing order: dizziness, drowsiness, headache, temporary eyesight, poor memory, and so on. Majority of the chronic effects mentioned by the rice wine drinker are: gastritis, liver disease, and liver cirrhosis.
(67) Frequency of Hepatitis B and C Infections and Drug Overdose Among Homeless Intravenous Drug Abusers in Shiraz, Iran

Afshari $\mathrm{R}^{1}$, Nabavi $\mathrm{A}^{2}$, Afshari $\mathrm{R}^{2}$.

${ }^{1}$ Medical Treatment Affairs Department, Shiraz University of Medical Sciences, Shiraz, Iran.

${ }^{2}$ Medical Toxicology Centre, Mashhad University of Medical Sciences, Mashhad, Iran

Introduction: Addiction and drug abuse is common in Iran. This study aimed to evaluate the seroprevalence of Hepatitis $\mathrm{C}$ virus (HCV) and Hepatitis $\mathrm{B}$ virus (HBV) infections among most high-risk subjects.

Methods: Subjects included 189 arrestees of a police crackdown with history of drug injection in March 2008. HBs antigen and HCV antibody were assessed in these homeless men as well as the attributed risk factors using a questionnaire. Results: Mean (SD, min-max) age of the participants was 38 (11, 19-68). Positive HCV and HBV infections were observed in 64 and $4 \%$ of study subjects, respectively. Thirty one percent recalled at least one episode of drug overdose.

Conclusion: Homeless cases with overdose or police arrests should be evaluated for liver-related viral infections. Mild drug overdoses are much more common than hospital-based reports.

\section{(68) Methanol Poisoning in a Pregnant Woman: a Case} Report

Seyed Reza Mousavi,Afsaneh Deh Bozorgi.

Medical Toxicology Research Center, Imam Reza Hospital, Faculty of Medicine, Mashhad University of Medical Sciences, Mashhad, Iran

Introduction: The toxic alcohols: methanol, ethylene glycol, and isopropyl alcohol are widely available in household and commercial products that are intentionally abused as ethanol substitutes. Although isopropyl alcohol is relatively benign, both methanol and ethylene glycol have serious, sometimes fatal, effects. Because of the widespread availability of these agents and their potential toxicity, toxic alcohol ingestion should be considered when a patient presents with a history or clinical picture consistent with such ingestion, associated with acidosis and serum chemistry with a widened anion or osmolar gap.

Materials and Methods: This study is a case report of a 33year-old woman 18 weeks pregnant that had been drinking some liquid by a suicidal attempt.

Results: First blood methanol level measurement was 35 $\mathrm{mg} / \mathrm{dl}$. She underwent hemodialysis for $2.5 \mathrm{~h}$. After hemodialysis, the patient was fully alert and able to verbally 
communicate. At this time, severe visual loss was reported by the patient. Both pupils were dilated without any reaction to light. Sonography for fetal evaluation revealed: oligohydramnios and no fetal heart sound. The patient underwent elective abortion.

Conclusion: Methanol is not only toxic for the mother but also is highly toxic and lethal for fetus.

(69) Nasal and Hand Staphylococcus aureus Carriage Rate Among Health Care Workers in Toxicological Intensive Care Unit of Loghman Hakim Hospital, Tehran, Iran

Haleh Talaie ${ }^{1,2}$, Sepideh Kamalbeik ${ }^{3}$, Arezou Mahdavinejad ${ }^{3}$. ${ }^{1}$ Toxicological Research Center, Loghman-Hakim Hospital, Shahid Beheshti University of Medical Sciences, Tehran, Iran. ${ }^{2}$ Center for Clinical Excellence, Shahid Beheshti University of Medical Sciences, Tehran, Iran.

${ }^{3}$ Microbiology Division, Islamic Azad University_ Fars Science and Research Branch, Shiraz, Iran

Background: One of the predominant microorganisms in several reports of nosocomial infections is Staphylococcus aureus, and many times in a day, this is carried by the health care workers. Present investigation was conducted to assess the nasal and hand carriage rate of $S$. aureus amongst medical personnel of Loghman Hakim Hospital's Toxicological Intensive Care Unit (TICU).

Methods: All health care workers of TICU $(n=70)$ were recruited in this study. A questionnaire with demographic information, work category, duration of working in the (TICU) and history of infection by S. aureus was completed by one trained nurse of ward. A total of 140 swabs (nasal $=70$, hand $=70$ ) were collected from personnel in TICU to screen for hand and nasal $S$. aureus colonization following standard protocol. Isolates with both inhibition zone diameter $\leq 13 \mathrm{~mm}$ around oxacillin disc and $\leq 21 \mathrm{~mm}$ around cefoxitin disc were considered methicillinresistant S. aureus (MRSA) strains. Also, we used KOH preparation technique to identify hyphae from the samples.

Results: Of the health care workers, $18(25.7 \%)$ were carriers of $S$. aureus. Methicillin-resistant $S$. aureus was found in $6(8.6 \%)$ subjects. About $5(7.1 \%)$ of the samples showed presence of yeasts.

There was no significant difference between the sexes $(p=$ $0.075)$, age $(p=0.080)$, work category $(p=0.100)$, and duration of working in the TICU $\leq 5$ and $>5$ years $(p=0.875)$ with regard to the positive and negative carriage of $S$. aureus (nasal and hand).

Conclusion: It is concluded that HCW's transmission of $S$. aureus has to be a concern. Although, more studies are required to examine the relationship between MRSA nasal or hand colonization status of a $\mathrm{HCW}$ and subsequent MRSA infection in patients
(70) Pattern of Acute Adult Poisoning at a Tertiary Care Hospital in the Western Province Sri Lanka: a Retrospective Study

Pradeepa Jayawardane, Inoka Wijegoonawardene, Tharangi Kodikara, S D Jayaratne.

Faculty of Medical Sciences, University of Sri Jayewardenepura, Nugegoda, Sri Lanka

Background: Though the epidemiology of acute poisoning has been extensively studied in agricultural areas of Sri Lanka, it has not been studied well in other parts of the country. The objective of this study was to describe the pattern of acute poisoning in a tertiary care teaching hospital in the Western Province of Sri Lanka.

Methods: This study was performed as a retrospective crosssectional study in Colombo South Teaching Hospital (CSTH), Western Province, Sri Lanka. The bed head tickets of the patients admitted to the medical units of CSTH from 1 January 2009 to 31 December 2009 were retrieved. Patients admitted to pediatric units and with snake bites and food poisoning were excluded.

Results: From 1 January 2009 to 31 December 2009, 92,668 patients were admitted to CSTH and $1.27 \%$ $(1,178)$ were admitted following acute poisoning. We were able to collect data on $70.46 \%$ of the patients (828). Male to female ratio was 37:63. Thirty-nine percent (325) of the patients were young, in the age category $21-30$ years. The commonest cause of poisoning was due to medicinal drug overdose $63.52 \%$ (526) of which paracetamol overdose accounted for $37.6 \%$ (311). Organophosphate accounted for $11.11 \%$ (92). Mean duration of hospital stay was 2.49 ( $\mathrm{SD} \pm 4.97$ ) days. Only $0.857 \%$ (7) needed intensive care management; of that, 6 were due to OP poisoning. Activated charcoal has been administrated to $71.86 \%$ (595) and gastric lavage has been performed in $65.2 \%$ (540). The mean time taken to perform gastric lavage (from ingestion) was $4.06(\mathrm{SD} \pm 5.22) \mathrm{h}$ with the mean time taken to administer activated charcoal from ingestion was $4.06(\mathrm{SD} \pm 5.239) \mathrm{h}$.

Discussion: The pattern of poisoning observed in Colombo South Teaching Hospital is significantly different to the pattern seen in agricultural areas of the country where organophosphate poisoning predominates.

\section{(71) Prices of Illicit Drugs in Mashhad, Iran from 1981} to 2011

Salahi-Motlagh $\mathrm{L}^{1}$, Safaee $\mathrm{M}^{1}$, Afshari $\mathrm{R}^{2}$.

${ }^{2}$ Medical Toxicology Center, Iman Reza Hospital, Mashhad University of Medical Sciences, Mashhad, Iran.

Introduction: Overdoses with illicit drugs are common in Iran. Many determinants have been proposed to model the pattern of dramatic changes in the types of drugs in the past 
decades. To date, no study has been performed to investigate the contribution of the prices of illicit drugs in this country. Methods: A questionnaire was developed and 220 cases from three rehabilitation centers were studied. Prices were presented in US dollars. The value of local currency has changed frequently in this period.

Results: Crude prices of opium (Theryak) in dollars have gradually decreased from 1981 to 2011 . The price of crystal heroine, which was introduced in this area in 2002, has declined more rapidly. Similarly, the price of methamphetamines (locally called Shisheh) has declined dramatically in recent years.

Conclusion: The pattern of price change for crystal heroin and methamphetamines are rather more dramatic than opium in this area. This might be a reflection of local production of synthetic drugs.

(72) Reduction of Serum Calcium Level by Sub-acute Intravenous Phenytoin Administration

Shafahi $\mathrm{A}^{1}$, Dadpour $\mathrm{B}^{2}$, Afshari $\mathrm{R}^{3,4}$, Moshiri $\mathrm{M}^{5}$.

${ }^{1}$ Mashhad University of Medical Science (MUMS), Imam Reza (P) Hospital, Mashhad, Iran.

${ }^{2}$ Medical Toxicology Centre, Mashhad University of Medical Science (MUMS) Imam Reza(p) Hospital, Mashhad, Iran.

${ }^{3}$ Education Development Centre, Mashhad University of Medical Sciences, Imam Reza (p) Hospital, Mashhad, Iran.

${ }^{4}$ Medical Toxicology Centre, Mashhad University of Medical Sciences, Imam Reza (p) Hospital, Mashhad, Iran.

${ }^{5}$ Department of Pharmacodynamics and Toxicology, School of Pharmacy, Mashhad University of Medical Sciences, Mashhad, Iran

Introduction: Hypocalcemia resulting from long-time treatment with Phenytoin $(\mathrm{Ph})$ has been known and it is suspected to be related to catabolism acceleration of vitamin $\mathrm{D}$. But there are not any reports of hypocalcemia following acute or sub-acute administration of $\mathrm{Ph}$ and we found it in sub-acute administration.

Methods: We evaluated retrospectively 1,814 documents of patients who had been admitted in Imam-Reza hospital of Mashhad since April 2010 to April 2011, and had received intravenous $\mathrm{Ph}$. Sixty one cases had including criteria: checked serum calcium level before (pre-Ca) and after (post-Ca) treatment and normal calcium level before commencing administration. We extracted the following data: age, sex, underlying diseases, diagnosis, sodium, potassium, total albumin, pre-Ca and post-Ca

Results: All patients had received intravenous $\mathrm{Ph}$ for 5 days. Post-Ca of 36 (59 \%) cases had reduced in comparison to pre$\mathrm{Ca}$ (mean $=1.13 \pm 0.1 \mathrm{mg} / \mathrm{dl} ; p<0.001)$, and 30 cases $(49.2 \%$ ) had become hypocalcemic (post-Ca $<8.5 \mathrm{mg} / \mathrm{dl}$ ). Differences of pre-Ca $[8.8(0.7,7.0-10.0) \mathrm{mg} / \mathrm{dl}]$ and post-Ca means $[8.5$
$(1.1,5.9-10.6) \mathrm{mg} / \mathrm{dl}]$ of all 61 cases were significant $(p=$ $0.023)$. The mean value of total albumin, sodium and potassium of all 61 cases were $3.3(0.4,2.4-4.3) \mathrm{g} / \mathrm{dl}, 140$ (5.6, $129-157) \mathrm{mEq} / \mathrm{L}$ and $4.4(0.8,3.0-6.2) \mathrm{mEq} / \mathrm{L}$, respectively, and there were not any significant differences in hypocalcemic and normocalcemic patients.

Conclusion: Sub-acute administration of $\mathrm{Ph}$ could reduce serum calcium. This may be due to the interaction of $\mathrm{Ph}$ with vitamin D metabolism. We recommend checking serum calcium during and after $\mathrm{Ph}$ therapy. Caution should be taken in the case of high-risk patients.

\section{(73) Relationship Between Pulmonary Function Tests and Clinical Findings in 43 Patients with Delayed Complications of Sulfur Mustard Poisoning}

Emadodin Darchini, Mahdi Maleknejad, Morad Bavandi and Mahdi Balali-Mood.

Medical Toxicology Research Center, Faculty of Medicine, Mashhad University of medical sciences, Mashhad, Iran.

Introduction: The most common complications of sulfur mustard (SM) poisoning have been observed in the respiratory tracts. Pulmonary function test (PFT) is a useful tool for diagnosis of the obstructive and/or restrictive lung diseases and their severities. It was aimed to find out relationships between PFT and clinical manifestations in patients with delayed complications of sulfur mustard poisoning.

Methods: After obtaining the informed consent, 43 veterans with more than $25 \%$ disabilities due to SM poisoning and 26 healthy controls were studied. Clinical examination was performed by an experienced chest physician and PFT was done by an experienced technician. Statistical analyses were carried out by means of SPSS 16 .

Results: The mean age of patients was 50.6 years and mean disability $47.7 \%$. Triad of chronic cough, dyspnea and expectoration were the most common symptoms which were recorded in $88.2,88.2$ and $64.7 \%$ of the patients, respectively, while it was $7.7,11.5$, and $7.7 \%$ in the control group. Twenty-one patients $(48.8 \%)$ who had the triad symptoms were overweight $(\mathrm{BMI}>25)$ and there was a significant correlation $(p<0.05)$ between the two variables. PFT abnormalities were much higher $(44.1 \%)$ in the patients than in the controls (11.5\%). Mixed pattern among the veterans raised significantly with higher disability percentages and there was a significant positive correlation $(p<$ $0.01)$ between them. We also observed a significant reverse correlation $(p=0.013)$ between the disability percentages of the patients and their FEV1/FVC.

Conclusion: Triad of cough, dyspnea and sputum were the main symptoms and the most impaired PFT was mixed obstructive and restrictive pattern. Correlations between them and BMI were significant. 
(74) Snakes in the Northern part of Bangladesh: SpeciesSpecific Identification

Fazle Rabbi Chowdhury ${ }^{1}$, Md. Shafiqul Bari ${ }^{1}$, S.M. Shamsul Ahsan ${ }^{3}$.

${ }^{I}$ Department of Medicine, Sylhet M.A.G. Osmani Medical College, Sylhet, Bangladesh.

${ }^{2}$ Medical officer, Mother and Child Welfare Center, Khulna, Bangladesh.

Objectives: The primary objective was to identify and report the venomous snake species that are available in the north-eastern part of Bangladesh. Site and location of bite, lag period between bite and hospitalization and outcome of treatment were also analyzed as secondary objectives.

Methods: This was an observational study done from July 2010 to July 2011 in the Department Of Medicine, Sylhet MAG Osmani Medical College Hospital. We received a total of 36 snake bite cases (both venomous and nonvenomous) but only those cases where offending snake were brought to hospital (dead or alive) were enrolled. Zoologist's opinion was taken to identify the species. Only five patients fulfilled the criteria and were finally enrolled. Data were presented as proportion and percentage.

Results: Mean age of cases was 29+2 SD years with $100 \%$ male. Three (60\%) snakes were krait (two Bungarus fasciatus (banded krait) and one Bungarus caeruleus (common krait)) and two were green pit viper Trimeresurus albolabris. Left foot was affected in $60 \%$ cases followed by right foot $(40 \%)$. The highest lag period between bite and hospitalization was $540 \mathrm{~min}$ and lowest was 90 min with an average of $282 \mathrm{~min}$. The most common location was in a narrow village road $(60 \%)$ followed by forest $(40 \%)$. Four snakes were brought dead and one alive (Trimeresurus albolabris). Three patients received traditional treatment in the form of herbal product before admission. Duration of hospital stay was $2-5$ days with a successful outcome in all patients.

Conclusion: The estimated incidence density of snake bite is around 623.4/100,000 person years in Bangladesh. So far, specific species were identified and reported from southern (Khulna and Barisal division) and eastern (Chittagong division) part of our country but not from the northern (Sylhet division) part. Species identification is important to get an idea on local distribution of snakes in Bangladesh.

\section{(75) Successful Treatment of Body Packers with Many Packages}

Ali Khoshkar ${ }^{1}$, Babak Mostafazadeh ${ }^{2}$.

${ }^{1}$ Department of Surgery, Loghman Hakim Hospital, Shahid

Beheshti University of Medical Sciences, Tehran, Iran.
${ }^{2}$ Department of Forensic Medicine and Clinical Toxicology, Loghman Hakim Hospital, Shahid Beheshti University of Medical Sciences, Tehran, Iran.

During the last decade, increase rate of drug trafficking and strict customs, have led the smugglers to attempt various methods. One of these methods of illicit drug smuggling is body packing. Body packers are people who illegally carry drugs, mostly cocaine as well as opium and/or heroin, concealed within their bodies. The packets are inserted in the mouth, rectum, or vagina in order to get across borders without being detected. The smuggling of drugs by internal concealment of these illicit goods is a growing problem for custom services and law enforcement officials, not to mention risks for the carrier from a medical point of view. Individuals engaged in such activities are called bodypackers. Here, we present 11 cases of body-packers admitted to our hospital in a period of $24 \mathrm{~h}$. Radiological examination including plain abdominal radiographs and CT scan images demonstrated the presence of multiple enteric packages. Non-surgical conservative evacuation of bowel contents was induced by the administration of laxatives. Analysis of the packages revealed crack and cocaine as concealed drugs.

(76) The Effect of Filgrastim on Myocardial Injury Following CO Poisoning: an Experimental Study in Rat

Hashemzaei M., Mohamadpour A. H., Imenshahidi M., Moalem S. A., Abnous, KH.

Department of Pharmacodynamics and Toxicology, School of Pharmacy, Mashhad University of Medical Sciences, Mashhad, I.R. Iran

Background: Carbon monoxide (CO) is an odorless, colorless, non-irritating gas that is produced within inefficient combustion of fossil fuels and also is the leading cause of death by poisoning in the USA. CO poisoning has many different effects on the heart. One of these effects is cardiomyocyte apoptosis. Granulocyte colony-stimulating factor (G-CSF) is the cytokine that can mobilize bone marrow stem cells and promote their differentiation to blood cells. In addition, G-CSF stimulates many anti-apoptotic pathways.

Methods: Rat model of animals were influenced by $\mathrm{CO}$ $3,000 \mathrm{ppm}$ poisoning. Evaluation of apoptosis was performed using caspase- 3 assessment by fluorometry and terminal deoxynucleotidyl transferase dUTP nick end labeling (TUNEL), and necrosis was assessed by hematoxylin/eosin staining. We here examined whether the G-CSF treatment after CO poisoning has beneficial effects or not.

Results: Caspase-3 activity assessment by fluorometry and TUNEL assessment confirmed that G-CSF $100 \mu \mathrm{g} /$ 
$\mathrm{kg}$ for four consecutive days after $\mathrm{CO}$ poisoning can significantly reduce apoptosis in rat cardiomyocytes. It was shown that G-CSF can significantly reduce caspase-3 activity and TUNEL-positive cells in treated groups in comparison with control group. Many other studies have clarified the effects of G-CSF after acute myocardial infarction and ischemia/reperfusion ( $\mathrm{I} / \mathrm{R})$ and have proved that G-CSF can ameliorate apoptosis following $\mathrm{CO}$ poisoning. In the histological findings, no statistically significant differences between control and treatment groups were shown.

Conclusions: G-CSF can reduce cardiomyocyte apoptosis after $\mathrm{CO} 3,000 \mathrm{ppm}$ poisoning in rat heart. But it had no effect on pathological findings.

\section{(77) Trismus Induced by Fluoxetine and Citalopram}

Mozhgan Amini ${ }^{1}$, Seyyed Hamzeh Hoseini.

${ }^{1}$ Medical Toxicology Center, Iman Reza Hospital, Mashhad University of Medical Sciences, Mashhad, Iran.

Case Presentation: The patient is a 35-year-old married lady with moderate major depressive disorder diagnosed according to the criteria from the DSM IV-TR diagnostic criteria. She was suffering from depressed mood, loss of appetite, hopelessness, suicidal ideation, anhedonia, and anergia for the last 3 months, and these symptoms were causing intense suffering and negatively affecting her job and interpersonal relations. She had a past history of mild depression 10 years ago, which was treated by lorazepam $1 \mathrm{mg}$ daily. The patient, however, had no history of concomitant use of any other medications, previous use of neuroleptics, present medical illness, brain problems, substance abuse, or other conditions that may have contributed to this adverse reaction. Therefore, pharmachological treatment with fluoxetine $(20 \mathrm{mg} /$ day $)$ was started. After a few doses in the first week of treatment, she complained of pain on eating meals. Her jaw was stucked and she was unable to talk and eat food. Her symptoms were worse at the end of the day, but there were no sign of bruxism or any dystonic symptoms or akathisia. With no improvement after changes in the time of medication intake or with dose fractionation, the drug was interrupted because of this side effect, which was resolved after the discontinuation of fluoxetine. After the interruption of fluoxetine, citalopram $20 \mathrm{mg} /$ day was tried. After the first dose of citalopram she complained of the same symptoms again. For control of depression, bupropione was started, with good response and no return of trismus.

Discussion: Movement disorders induced by selective serotonine reuptake inhibitors (SSRIs) are a very rare adverse effect and there are only a few reports available. Reduction of the dose may be a therapeutic alternative, although many patients benefit more from the removal of medication or from another antidepressant, as was the case of present report. Therefore, this case illustrates the importance of being aware of rare but possible occurrence of trismus as an adverse effect of the SSRIs. Future studies on the pathophysiology of this adverse effect seem to be necessary.

\section{(78) Venous Thrombosis Following Intravenous Injection of Household Bleach}

Seyed Hesam Rahmani ${ }^{1}$, Sajjad Ahmadi ${ }^{1}$, Samad Shams Vahdati $^{1}$, Hossein Hasssanian Moghaddam ${ }^{2}$.

${ }^{1}$ Tabriz University of medical sciences, Tabriz, Iran.

${ }^{2}$ ShahidBeheshtiUniversity of Medical Sciences, Tehran, Iran

Sodium hypochlorite (NaCLO) is used extensively as a disinfectant or bleaching agent. Most studies describe ingestion or inhalation route of this product with rare complication and fatalities. Despite global daily exposures, data about bleach injection are limited. Here, we report intentional infusion of $20 \mathrm{ml}$, sodium hypochlorite $5 \%$ diluted in $500 \mathrm{ml}$ normal saline into right cubital vein ( $1.5 \mathrm{~h}$ prior to admission) in 30 year-old previously physically healthy man with a history of schizophrenia since 10 years ago. Clinical manifestation included local pain and edema. There were no laboratory abnormalities in the patient. Doppler sonography revealed thrombosis in superficial (antecubital and basilic) veins. Limb elevation, warm compress, and ibuprofen relieved the pain and edema after 3 days. We hypothesize that thrombosis may be triggered by direct damage of endothelial response resulted from potent oxidant effects of sodium hypochlorite. Based on this theory, storage and depletion of glutathione has an important role to show the amount of endothelial response following this oxidative stress. Administration of antioxidants glutathione in severe cases might be beneficial.

\section{(79) A Severe Case of Haff Disease After Eating Buffalo Fish in Northern California}

Steven R. Offerman, Ethan E. Bodle.

Kaiser Permanente Northern California, Antioch, CA, USA

Introduction: Toxic contamination of fish may compromise safety. Haff disease is a rare syndrome characterized by rhabdomyolysis after consumption of cooked, freshwater fish.

Case Report: A healthy 42-year-old female presented to the ED $3 \mathrm{~h}$ after eating fried buffalo fish (Ictiobus cyprinellis). This was her first time eating this fish which was purchased from a health food grocery in Berkeley, CA, USA. Her symptoms began while eating the fish, described as diffuse muscle aches followed within minutes by generalized muscle fatigue, most pronounced in her proximal lower extremities. She also described fingertips and peri-oral paresthesias. Vital signs were normal. Her physical exam was notable for diffuse muscle tenderness and difficulty 
standing. Admission $\mathrm{CBC}$ and chemistries were normal, but serum CK was 12,777 U/L. ALT and AST were 268 and $1,431 \mathrm{U} / \mathrm{L}$, respectively. She was treated with IV fluids, bicarbonate, and oral $N$-acetylcysteine. The serum $\mathrm{CK}$ peaked at 76,364 U/L approximately $20 \mathrm{~h}$ after fish exposure. Her creatinine remained normal. Over the next 5 days, CK slowly resolved and she was discharged. At 6 weeks follow-up, she still complained of diffuse muscle weakness, but CK levels remain normal.

Discussion: This is a healthy female who developed severe Haff disease after ingesting buffalo fish. Haff disease, first reported in 1924, describes rhabdomyolysis following consumption of certain cooked, freshwater fish. It is felt to be caused by a yet unidentified toxin. There have been 23 cases reported in the USA, 12 of these related to consumption of buffalo fish. Our case is unique due to the immediate onset of symptoms and the extremely high serum CK levels. Our patient never developed renal manifestations of her severe rhabdomyolysis.

Conclusion: Haff disease may result in the rapid onset of severe rhabdomyolysis. Aggressive treatment with fluids and bicarbonate prevented renal damage in this case.

\section{(80) An Observational Study of Snake Bites Presented to a Tertiary Care Centre in India}

\section{Bhalla A, Shafiq N, Sharma N, Singh S. \\ Postgraduate Institute of Medical Education and Research, Chandigarh, India.}

Introduction: Venomous bites constitute an important cause of hospitalisation and mortality in developing nations. In the present study, we discuss the salient features of snake bite cases presented to our centre.

Subjects and Methods: The study was conducted prospectively in the Emergency Medicine Department of our hospital. All the patients presenting with snake bites from January to December 2008 were included in the study. Data extracted included demographic details like age, sex, marital status, residence, educational qualification, occupation and monthly income. The time of snake bite, time of hospital arrival, lag time in reaching the hospital and reasons for the same, and mode of transportation were noted down. The patients/attendants were asked about first aid received, the details of first aid, timing and by whom. The records of any outside treatment, setting and qualification of treating doctor were checked. The details of indoor treatment, door to needle time (defined as time from hospital arrival to administration of first treatment), requirement of antidote and the dose delivered were recorded. Finally, the duration of hospital stay and recovery status was noted. Data was presented as numbers, percentages, mean $\pm \mathrm{SD}$ and median (interquartile range (IQR)). Logistic regression and univariate analysis were used to predict the effect of various confounding factors on the outcome and duration of hospital stay, respectively. $P$ value $<0.05$ was considered statistically significant. Results: Sixty-four patients presented with snake bite were: neurotoxic (35), vasculotoxic (9) and non-venomous (20). Ninety percent of the victims were males. Mean age was 29 years. Identical representation of the study population from urban and rural areas was noted. More than half of the patients were illiterate and were labourers. Seventy-five percent were bitten during their sleep and the rest during the early hours or late in the night. Proximal joint tourniquet application at home was done in $39 \%$ snake bite cases (25/ 64 ) and local incision was given in $20 / 64$ patients. First aid was mostly administered by some close relative living with the patient. The median (IQR) lag time in hospital arrival in snake bite cases was $3.9(2.2-7.3) \mathrm{h}$. Transportation time and referral from some other hospital were mainly responsible for delay in reaching the hospital. The mode of transportation was personal/private vehicles in majority. Around $70 \%(45 / 64)$ of patients with snake bite groups had received outside treatment by graduate/postgraduate doctors in private/ civil/government hospital settings. Outside treatment was mainly in the form of intravenous fluids and injectables. Only $10 \%$ of snake bite patients (6/64) had received anti-snake venom in the periphery but in inappropriate dose. The median (IQR) door to needle time was $35(30-52.5) \mathrm{min}$. Ten vials of anti-snake venom were given in (48/64) snake bite patients. Repeat dose was administered in only 30 patients having persistent neurological deficit or coagulopathy. Twenty of 35 patients with neuroparalysis required ventilation and 5 out of 9 with vasculotoxic bite required renal replacement. There was one mortality $(1.6 \%)$ due to cardiac arrest among all snake bite cases. None of the various confounding factors was found to significantly affect the outcome in snake bite cases. Out of the various determinants (for corrected model: $F=1.255 ; P=0.293$ ), only receipt of outside treatment showed a trend towards decreasing the duration of hospital stay although this also could not achieve statistical significance $(F=2.978 ; P=0.09)$.

Conclusion: Snake bite is an important cause of emergency admissions at our centre, but with improvement in management, the mortality associated is greatly reduced.

\section{(81) An Overview of the Types of Natural Toxins Poisoning Cases Reported to the National Poison Center for the Period of 2006 to 2009}

Azizan Haji Daud, Razak Hj Lajis, Adilah Mohd Ariff, Sa'ed H. Zyoud, Halilol Rahman, Sulastri Samsudin.

National Poison Center, University Science of Malaysia

Objective: Poisoning involving natural toxin (NT) is one of the national health problems in Malaysia that is often 
overlooked. The aim of the study is to describe the pattern of NT poisoning reported to the National Poison Center (NPC) in Malaysia over a 4-year period.

Methods: We reviewed and analyzed all calls registered by the NPC 24-h information and consultation service for the period between 1 January 2006 and 31 December 2009 focusing on the poisoning cases that involved NT. Age, gender, date of exposure, route of exposure, and type of NT poisoning were evaluated. SPSS version 15 was used for descriptive analysis of the data collected.

Results: During the study period, the NPC collected 292 telephone enquiries from the emergency departments in Malaysia regarding NT poisoning. Most calls were made by medical doctors $(98.6 \%)$ followed by other health professional $(1.0 \%)$ and public $(0.3 \%)$. NT poisoning occurred mostly in males (65.4\%). Snake bite accounted for 142 cases $(48.63 \%)$, followed by spider/scorpion/centipede poisoning in 41 cases $(14.04 \%)$, insects poisoning in 9 cases $(3.08 \%)$, jelly fish poisoning in 11 cases $(3.77 \%)$, other marine poisoning in 17 cases (5.82\%), plant poisoning in 17 cases $(5.82 \%)$, bacteria/fungi/algae poisoning in 48 cases $(16.44 \%)$, other NTs in 6 cases $(2.05 \%)$, and 1 case $(0.34 \%)$ of unknown NT poisoning. The number of poisoning calls from the emergency departments regarding NT poisoning was observed to have increased annually from 45 calls (15.42\%) in 2006 to 107 calls (36.64\%) in 2009 ( $p<$ $0.001)$.

Conclusion and Recommendations: There has been an increase in the number of calls received by the NPC involving NT poisoning. Based on the available data from the reported cases, animal toxins constitute $75 \%$, and other toxins comprise only $25 \%$. Further study is required to determine more accurately the degree of morbidity and mortality related to poisoning by NT.

\section{(82) Comatose Children Poisoned by Persian Silk Tree Seed: a Case Report}

Seyed Reza Mousavi, Hamid Khosrojerdi.

Medical Toxicology Research Center, Imam Reza Hospital, Faculty of Medicine, Mashhad University of Medical Sciences, Mashhad, Iran

Introduction: Persian silk tree, a variety of Albizia julibris$\sin$ (mimosa), is native from Iran to central china. Julibrissin is a corruption of the Persian word gul-i abrisham which means "silk flower". It is highly valuable in Chinese medicine as it has the most powerful properties for treating stress and anxiety depression. Symptoms of mimosa toxicity due to ingestion include stomach upset, ataxia, and lethargy in horses. All parts of this tree may cause severe discomfort if ingested. Ingestion of its seed is one of the strongest hallucinogenic herbal medicines.
Materials and Methods: This is a case report of three children $(3.5,3.5$, and 4.5 years old) who ingested accidentally unknown amounts of Persian silk tree seed.

Results: All of them showed altered level of consciousness for about 5 to $8 \mathrm{~h}(\mathrm{DCS}=7)$. Laboratory tests in our patients were in normal ranges and only QTC in ECG of one was more prolonged than normal $(0.46 \mathrm{~ms})$. One of children had obvious flushing in her checks that continued for about $6 \mathrm{~h}$. Conclusion: Although, Persian silk tree seed rarely can cause toxicity, but with regard to its widespread, it may cause poisoning, especially in children who ingested it accidentally. It can cause coma, hallucinations, convulsions, and tremor, but needs more clinical trial in animals for investigation of adverse effects.

(83) Comparative Study of Merrem's Hump-Nosed Pit Viper (Hypnale hypnale) Bites Between Two Climatic Zones (Wet vs Dry Zone) in Sri Lanka

Kalana Maduwage ${ }^{1}$, Chanaka Rathnayake ${ }^{2}$, SAM Kularatne ${ }^{2}$.

${ }^{1}$ Department of Biochemistry, Faculty of Medicine, University of Peradeniya, Sri Lanka.

${ }^{2}$ Department of Medicine, Faculty of Medicine, University of Peradeniya, Sri Lanka

Sri Lanka is a tropical island with great diversity in geography and climatic conditions that divides the country into three climatic zones: wet, intermediate and dry. Of the three species of Hypnale found in Sri Lanka, Hypnale hypnale is widely distributed and is responsible for most of the snake bites. The effects of different climatic conditions on the pattern of envenoming remain as an unanswered question. This study aims to investigate possible differences of $H$. hypnale bites between wet zone and dry zone using authentic systematically collected data from these regions. We collected 39 (wet zone) and 33 (dry zone) H. hypnale bites over a 1-year period from two major hospitals representing each zone. Offending snakes in all cases were identified and measured and their sexes were determined. In the wet zone, 28 (66 \%) of $H$. hypnale bites occurred from August to October whilst in the dry zone, 20 (60\%) bites occurred from January to April corresponding to the monsoon rains. In both zones, majority of bites occurred in the surroundings of compounds; however, $6(18 \%)$ and 1 (3\%) bite occurred indoor in dry and wet zone, respectively. Occurrence of local necrosis is higher in dry zone bites, 12 (36\%), compared to $5(13 \%)$ in the wet zone. Six $(18 \%)$ had coagulopthy in dry zone bites compared to $1(3 \%)$ in wet zone. Development of neurotoxicity was documented in a patient from dry zone. We assume that the higher incidence of $H$. hypnale bites is related to the monsoon rains than to the climatic zones. However, differences in clinical manifestations such as severe local envenoming, coagulopathy and neurotoxicity were observed as they occurred more frequently in the dry 
zone bites. Even though, acute renal failure is a known complication of $H$. hypnale envenoming, this study suggests its infrequency of occurrence irrespective of climatic zones.

(84) Isometrus maculatus and Other Scorpion Stings That Presented to an Emergency Department in Singapore

Hock Heng Tan, Rupeng Mong.

Department of Emergency Medicine, Changi General Hospital, Simei, Singapore

Introduction: This is a retrospective study of patients with scorpion stings presented to a local emergency department from 2004 to 2009 . The characteristics and clinical features of stings by scorpions found locally, including that of the venomous scorpion Isometrus maculatus, are described.

Materials and Methods: Patients with scorpion stings were identified by searching through cases in the emergency department with the ICD codes E905 and 906, inpatient records, and those referred to the hospital's toxicology service. Their records were traced and the clinical data were double extracted and analyzed.

Results: A total of 12 cases were identified. The main symptom was pain, with other common complaints being swelling and numbness. Some patients also had tachycardia, hypotension, or hypertension. Most cases responded to supportive treatment at the emergency department and were discharged after a brief period of observation. Only two patients required admission for complications from the scorpion sting. Both of these cases were stings that occurred in neighbouring countries. No serious adverse effects were reported in all cases. Three cases were identified to have been stung by the scorpion I. maculatus (striped scorpion) from the family Buthidae. The identities of the scorpions were confirmed by photo identification with the Toxin Research Institute in Singapore. All three patients had symptoms of pain and numbness around the sting site. The main neurological deficit was numbness, which radiated from the site of injury. Resolution of the neurological deficits took 1 to 2 days.

Conclusion: Scorpion stings are infrequent presentations to the emergency department. Most local scorpion stings cause local reactions which are self-limiting and respond well to supportive treatment. Stings from the species I. maculatus can however cause neurotoxicity, although this is mild and usually resolves after a few days.

\section{(85) Networking for Antivenom}

Ahmad Tajuddin Bin Mohd Nor, Ranjini Sivaganabalan, Norafizan Binti Mohd Ali.

Ministry of Health, Putrajaya, Malaysia
Introduction: Antivenom for Malaysian snake bites is widely available and easily accessible in our country. Most countries stock antivenom for animal bites and stings which are native to their country or region only. Zoos are required to stock specific antivenom for all venomous species of snakes not native to their region. What happens when a patient is bitten by a snake not native to our country? Antivenom for such snakes is unfortunately not easily available. How do clinicians obtain antivenom for snake bites not native to their country or region?

History and Progress: A 21-year-old gentleman presented to the Emergency Department after sustaining a bite from a green diamond back rattle snake. He presented with full signs of envenomation and was immediately resuscitated. Local polyvalent antivenom was given to the patient but there was no improvement and the patient's condition worsened. The patient was transferred to the Intensive Care Unit where he continued to deteriorate in spite of best efforts. Attempts to obtain the necessary antivenom were made in earnest and finally the antivenom was obtained from a neighbouring country. The patient was given the relevant antivenom and immediately improved. He was subsequently discharged home well.

Conclusion: Good networking and quick transfer of the antivenom with help from the necessary officials helped save the patient's life. Stocking antivenom for snakes not native to the region is not cost-effective. Global networking among clinicians and pharmacists can help facilitate treatment of patients suffering a similar fate.

\section{(86) Tetrodotoxin Poisoning in a Deadly Meal of Horseshoe Crab}

Che Nin Man, Razak Hj Lajis, Nurjuliana Mohd Noor. National Poison Centre, Universiti Sains Malaysia, Penang, Malaysia

Horseshoe crab (Carcinoscorpius rotundicauda) known locally as Belangkas can be found in shallow water along the Malaysian beaches. Although not commonly consumed by everyone, it is considered a delicacy to some sections of the population. The number of horseshoe crab is said to be fast depleting as it is also sought for its purported medicinal value. No incident of death from consumption of horseshoe crab has been reported until two incidents in Kota Marudu, Sabah, recently. The cases involved five people who had consumed a meal of Belangkas that resulted in one death. Sign and symptoms of tetrodotoxin (TTX) poisoning occurred within a few hours following consumption, more significantly for the victims who ingested the most. The victims developed oral numbness, shortness of breath, dizziness and respiratory depression $4 \mathrm{~h}$ after consumption. The age of the victims ranged from 24 to 72 years. Five urine 
and blood samples were received by the National Poison Centre for TTX analysis within a week of the incident. Due to the rapid metabolism of TTX, only urine samples were analysed and the levels were quantified according to the developed and validated GC-MS assay. TTX was detected in four of the five urine samples with the highest concentration in the urine sample of the deceased $(93.4 \mathrm{ng} / \mathrm{mL})$. The victim, a 72-year-old male died a few hours after admission to hospital. The other three victims survived (TTX, 1.3-17.3 ng/mL) despite experiencing various degrees of poisoning symptoms to TTX exposure. No TTX was detected in the fifth urine sample. Since there is no guarantee on the safety in the consumption of horseshoe crab, it would be beneficial for relevant authorities to make available information on the risks and hazards of consuming horseshoe crab and its by-products for public safety.

(87) The Effect of Mycotoxin Citrinin on Cardiac Development in Zebrafish (Danio rerio) Embryos

Ting-Shuan Wu, Biing-Hui Liu.

Chung Shan Medical University, Taichung City, Taiwan.

Citrinin (CTN), a fungal secondary metabolite from Penicillium and Aspergillus species, is frequently detected in wheat, corn, rice, and barley. Although CTN is considered to be a teratogen in rat, its effect on the early embryonic development is not clear. The zebrafish is considered as a good animal model for a toxicological study because of its short generation time and transparent embryos. In this study, zebrafish embryos were treated with CTN from 6 h postfertilization (6 hpf) or 24 to $120 \mathrm{hpf}$, and the LC50 values were found to be 15.3 and $16.6 \mu \mathrm{M}$, respectively. The morphology of CTN-treated embryos was heart tube malformation and yolk sac edema with red blood accumulation. With whole-mount immunostaining using MF20and S46 as probes, the formation of small hearts with abnormal looping showed a dosage-dependent manner in the 24-72 hpf CTNtreated embryos. Furthermore, the Tg (BMP4: EGFP), with fluorescence in heart chambers, was used to measure the heart shape and size after toxin treatment. When embryos were treated with $50 \mu \mathrm{M}$ CTN from 24 to $72 \mathrm{hpf}$, the distance between sinus venosus to bulbus arteriosus was significantly decreased to $78.7 \%$ of the control level. In addition, the areas of ventricle and atrium in CTN-treated groups were decreased to 51.5 and $49.8 \%$ of vehicle-treated groups, respectively. The heartbeats of $50 \mu \mathrm{M} \mathrm{CTN}$-treated embryos were also decreased from $154.9+4.8$ to $107.5+5.8$ / min, suggesting that CTN may trigger bradycardia in zebrafish embryos. On the other hand, we did not observe any morphological change of pronephric kidney in CTN-treated embryos; either glomerulus or pronephric tubes/ducts did not demonstrate apparent difference comparing to control groups. On the basis of these results, we suggested that CTN is able to induce cardiotoxicity in zebrafish embryos.

(88) Toxic Plants Poisoning Cases Reported to National Poison Center (2006-2009)

Azizan Haji Daud, Razak Hj Lajis, Adilah Mohd Ariff, Sa'ed H. Zyoud, Halilol Rahman.

National Poison Center, University Science of Malaysia, Penang, Malaysia

Introduction: Some plants have medicinal values whilst others may have toxic components. Poisoning regarding plants may occur due to intentional or unintentional consumption of the plants. These plants can be found both growing wild and in gardens, school compounds or public parks.

Objective: The aim of the study is to review the trend of cases involving plant poisoning reported to the National Poison Center (NPC) for the period of 2006-2009.

Methods: We analysed the calls registered by the NPC 24$\mathrm{h}$ information and consultation service for the period between 2006 and 2009 focusing on the poisoning cases that involved plants. Age, gender, date of exposure, route of exposure, and type of NT poisoning were evaluated. SPSS version 15 was used for descriptive analysis of the data collected.

Results: Data analysis showed that 17 poisoning cases caused by poisonous plants have been reported which contributed to $5.82 \%$ of the total poisoning cases caused by natural toxin. All calls were made by medical doctors. Plant poisoning occurred mostly in males $(58.82 \%)$. These plants include Datura sp. (four cases), Jatropha sp. (three cases), Antiaris toxicaria (three cases), Mitragyna speciosa (two cases) and one case of Caladium sp. It was observed that there was an increasing trend in the number of calls received.

Conclusion and Recommendation: There has been an increase in the number of calls received by the NPC involving plant poisoning. Although poisoning cases caused by plants are quite rare but it is still important to inform the public about the types or species of poisonous plants around us. Certain degree of precaution or awareness should be inculcated as danger posed by such plants can cause physical discomfort and mental stress. It could also lead to death. More national studies should be carried out regarding plant chemistry and toxicity.

(89) Evaluation of an Educational Intervention on Management of Pesticides Poisoning Among Pharmacists in Government Hospitals in Malaysia

De Silva WDAS, Awang R.

National Poisons Centre Malaysia, Universiti Sains Malaysia, Penang, Malaysia 
Background: Pharmacists of the government hospitals in Malaysia provide drug and poisons information to health professionals. Whether the extent of the content of the undergraduate curriculum on pesticides and other chemical poisoning is adequate is a concern. Analysis of calls received by the National Poisons Centre on pesticide poisoning indicates an increasing trend.

Objectives: The aim of this study is to evaluate the effectiveness of a clinical update on management of pesticide poisoning among pharmacists.

Methods: A 2-day workshop on clinical management of pesticides poisoning for pharmacists was conducted by the National Poisons Centre. Twenty six pharmacists from the government hospitals participated. Knowledge on pesticides poisoning, decontamination methods, antidotes, reference sources and attitudes on decontamination were assessed by pre- and post-assessment. Chi-square test was used for preand post-comparison.

Results: Post-evaluation revealed that, knowledge among participants was significantly increased on pesticides classification by $46 \%$ ( $p=0.004)$, pesticides responsible for poisoning in Malaysia by $35 \%(p=0.002)$, toxicity by $35 \%$ ( $p=$ $0.0012)$ and antidotes by $65 \%(p<0.001)$ compared to the baseline knowledge. Knowledge on indications to perform gastric lavage and activated charcoal was also significantly increased by $42 \%(p=0.002)$ and by $69 \%(p=0.003)$ consecutively. Attitudes towards administration of gastric lavage was changed favourably by $54 \%(p<0.001)$ and activated charcoal by $41 \%(p=0.013)$. Sixty-four percent of the participants strongly disagreed to consider forced emesis as the best GI decontamination method in poisoning before admission, compared to $30 \%$ in the pre-assessment $(p=0.048)$. Majority (76\%) strongly disagreed on giving various fluids to poisoned patients to neutralise the toxic effects at home compared to $30 \%$ in the pre-test $(p=0.0012)$.

Conclusion: Clinical update on management of pesticide poisoning is vital for pharmacists who provide poisons information.

\section{(90) Homicidal Paraquat Poisoning in Sri Lanka}

Professor Ravindra Fernando.

Department of Forensic Medicine and Toxicology, Faculty of Medicine, University of Colombo, Colombo, Sri Lanka

Paraquat is a dipyridylium quaternary ammonium salt that is used widely as an herbicide. Although cases of accidental and suicidal paraquat poisoning were documented in Sri Lanka, no cases of homicidal paraquat poisoning have been reported. One evening, a man who claimed to be from the Anti-Filaria Campaign visited a household. He said he had a list of names, whose blood tests performed earlier, were positive for filariasis. Then he gave a greenish blue liquid from a bottle to drink to the three family members present (mother and two daughters), whose tests he said were positive for filariasis. Within half an hour of consuming the liquid, all three developed gastro-intestinal symptoms and were admitted to the General Hospital, Colombo. The 53-year-old mother and the 25-year-old younger daughter died within $48 \mathrm{~h}$. The 29year-old elder daughter, who consumed only a mouthful of the 'medicine', died 13 days later. Analysis of the liquid showed it to be paraquat and the symptoms, signs and the clinical course of the patients were consistent with acute paraquat poisoning. The patient who survived for 13 days had characteristic proliferative fibrosing alveolitis of lungs. Investigations revealed that a family member was responsible for these homicides.

\section{(91) Poisoning in Bangladesh-DMCH Survey}

Ariful Basher ${ }^{1}$, Richard J Maude ${ }^{2}$, Robed Amin ${ }^{1}$,Aniruddha Ghose $^{3}$,HAM Nazmul Ahsan ${ }^{1}$, M Abul Faiz4.

${ }^{1}$ Dhaka Medical College Hospital, Dhaka, Bangladesh.

${ }^{2}$ Mahidol-Oxford Tropical Medicine Research Unit, Bangkok, Thailand.

${ }^{3}$ Chittagong Medical College, Chittagong, Bangladesh. ${ }^{4}$ Government of Bangladesh, Dhaka, Bangladesh.

Objectives: Poisoning is a major cause of morbidity and mortality worldwide. The number of chemicals used in industry, agriculture and medical profession is rising faster than it was anticipated. Consequently, misuse of pesticides, chemicals (harpic, nail polish and rat killer) and drugs (sedatives) has been observed. A large number of poisoning cases remains un-reported due to lack of information and awareness at community level. Therefore, the study has been designed to collect baseline information and assess magnitude of health problem that occurs due to poisoning. Methods: For all cases of poisoning from 1 April 2008 to 30 January 2009 from the Medicine and Paediatric Wards of Dhaka Medical College Hospital (DMCH), a study-specific data collection form completed for each case by a team of specially trained medically qualified research assistants. Details of presentation, social background and outcome were recorded.

Results: In a total of 5,932 cases of poisoning in DMCH, 2,108/5,929 (35\%) were females, 3,821/5,929 (65\%) were males. Median age was 25 years (IQR $19-35$ years). It is most common in the young but fatality was more observed between 60 and 75 ages. Peak was recorded in AugustNovember. Major substances were benzodiazepines (including deliberate/"street"; $36 \%)$, OPC/carbamate (12\%), snake/fish/insect, medications. Around $1 / 3$ were unknown substances. About $95 \%$ seek care from a government hospital. Suicidal attempt (43.8\%) due to family disharmony (37.7 \%) was the maximum cause. Besides, a large number were victimized by a stupefying agent (38.2\%). Overall mortality was $151 / 5,932$ (2.6\%); nevertheless, highest death 
was observed in herbal medicine poisoning ( $25 \%)$, then OP poisoning $(16 \%$ ), methanol poisoning (11\%) and copper sulphate poisoning $(9 \%)$. Major risk factors for mortality were: living in rural areas, Sanatan religion, illiteracy, being a farmer, suicide attempt, deliberate self-harm, and the following clinical evaluations-GCS $<9$, BP $<80 / 60$, HR $>100 /<60$, abnormal pupils (especially constricted or pinpoint), and not age, gender and marital status.

Conclusion: Multiple logistic regression is desirable to determine independent risk factors for mortality. Clinical scoring system should need to be done for predicting mortality. Detailed analysis should be made of $\mathrm{OCP} /$ carbamate poisoning management.

\section{(92) Protective Effects of Crocin on Diazinon-Induced} Subchronic Hepatotoxicity in Rat

Lari P, Hosseinzadeh H, Rashedinia M, Razavi M, Rashedinia M.

School of Pharmacy, Mashhad University of Medical Science, Mashhad, Iran

Diazinon is one of the most widely used insecticides in agricultural pest control. Previous studies have shown that diazinon causes hepatotoxicity, reactive oxygen species and apoptosis pathways might be involved in the toxicity of diazinon. On the other hand, crocin from saffron has hepatoprotective effect due to its anti-oxidant activity. In this study, we examined the protective effect of crocin on diazinon-induced subacute hepatotoxicity. Animals were initially divided into five groups. Corn oil, diazinon $(15 \mathrm{mg} / \mathrm{kg}$ per day, once a day in corn oil via gavage) and crocin $(12.5,25,50 \mathrm{mg} / \mathrm{kg}$ per day intraperitoneally) in combination of diazinon were given to male wistar rats $(n=6)$ orally for 4 weeks. The levels of malondialdehyde (MDA) as a marker of lipid per-oxidation and caspases-3, caspases- 8 and caspases- 9 as markers of apoptosis were evaluated. The result showed that crocin at a dose of $25 \mathrm{mg} / \mathrm{kg}$ was significantly effective in decreasing the elevated levels of MDA $(p<0.001)$. Our preliminarily results indicated the involvement of caspases in hepatotoxicity of diazinon and protective effects of crocin.

\section{(93) Review on Paraquat Poisoning in Malaysia After Lifting of Ban}

Sazaroni MR, Rahmat A, Sa'ed Z, Haslina H, Adilah MA, Asdariah M.

National Poison Centre, Universiti Sains Malaysia, Malaysia

Abstract Text:

Introduction: Paraquat is one of the most popular pesticides for weed control in Malaysia. Despite its high toxicity, it is widely used for its effectiveness. Due to frequent and serious poisoning involving this agent, the use of paraquat was banned in 2005 but was reintroduced into the market a year later and is currently available.

Objective: The aim of this study is to assess the outcome of the lifting of the ban and the occurrence of paraquat poisoning in the country.

Method: Poisoning cases referred to National Poison Centre (NPC), Malaysia for the period of 2005-2009 were analysed. Special emphasis was directed on herbicide cases involving paraquat exposure. Age, gender, ethnic, route of exposure, reason of exposure, and the number of paraquat calls during the ban and after its re-introduction were evaluated. SPSS version 15 was used for descriptive analysis of the data collected.

Result: A total of 278 calls involving paraquat were received during the period of the study. The cases mainly involved adult males $(68.4 \%)$ and common among Indians $(32.1 \%)$ compared to Chinese (22\%) and Malay (22\%). Suicide attempts were the most common $(73.8 \%)$ circumstances of exposure. Accidental paraquat poisoning mostly involved exposure through ingestion $(80.6 \%)$, followed by inhalation (12.3\%) and cutaneous (7.1\%).

The number of calls relating to paraquat exposure when it was banned was 67 (36 and 31 in 2005 and 2006 respectively). After its re-introduction, there was a marked increased in the number of cases: 39, 79 and 101 for 2007, 2008 and 2009, respectively.

Conclusion and Recommendations: There is an increment in the number of calls received by the NPC involving patients exposed to paraquat from year 2007 to 2009 following the lifting of the ban. This should prompt the Government of Malaysia to review the lifting of the ban. Poor enforcement of regulations on paraquat use has resulted in intentional poisoning involving the chemical. Knowledge on the proper storage and disposal of paraquat must be imparted to its users. Similar study on a national level should be undertaken to have a more comprehensive data on paraquat poisoning.

\section{(94) Upper Gastrointestinal Bleeding After Aluminum Phosphide Tablet Ingestion: a Case Report}

Bhalla A, Kochhar R, Sharma N, Singh S.

Postgraduate Institute of Medical Education and Research, Chandigarh, India

\section{Abstract Text:}

Introduction: Ingestion of aluminum phosphide as an agent of self-harm is associated with significant mortality. Some of the survivors end up with dysphagia, and multiple esophageal strictures have been documented in them. The exact cause of the strictures is not known but local impaction and 
exothermic injury to the esophageal mucosa have been implicated. We report a case of documented esophageal injury in a patient with aluminum phosphide poisoning presenting with upper GI bleed.

Case Report: A young male presented to the medical emergency in shock after alleged ingestion of two fresh tablets of aluminum phosphide $3 \mathrm{~h}$ prior to admission. The ingestion was followed by recurrent vomiting and giddiness. On examination, the patient was in shock with pulse rate of $120 / \mathrm{min}$ and unrecordable blood pressure. Peripheries were cold and clammy and JVP was raised. Ryle's tube was inserted and gastric lavage was done which revealed evidence of upper gastrointestinal bleeding. A central venous access was established and opening CVP was $20 \mathrm{~cm}$ of saline. Arterial blood gases revealed a pH of 6.9 with bicarbonate of 8 . He was started on dopamine, dobutamine and noradrenaline. Sodium bicarbonate was administered to correct metabolic acidosis. Magnesium sulfate was administered to control arrhythmias and $\mathrm{N}$-acetylcysteine was administered to reverse oxidative damage. The blood pressure improved to $90 \mathrm{mmHg}$ on treatment and metabolic acidosis reversed. Since there was evidence of upper gastrointestinal bleeding, endoscopy was performed which revealed areas of mucosal haemorrhage and ulcers in the mid-esophagus. The rest of the GI tract up to the second part of duodenum was normal. Proton pump inhibitors in high dose and sucralfate were administered. The patient improved and was discharged on the seventh day with an advice to follow-up in gastroenterology for esophageal injury.

Discussion: The tablet of aluminum phosphide is $18-20$ $\mathrm{mm}$ in diameter and can get lodged at sites of physiological narrowing in the esophagus. Once lodged, it releases phosphene in exothermic reaction locally and may result in a thermal esophageal injury akin to pill esophagitis. Our case highlights the acute effects of aluminum phosphide ingestion on esophageal mucosa and proves beyond doubt that acute esophageal ulcers due to a local injury to the esophageal mucosa can result in severe UGI bleeding and may be harbingers of late onset esophageal strictures.

Conclusion: Acute esophageal mucosal injury in the form of ulcers can lead to upper gastrointestinal bleeding in the acute phase and late onset dysphagia and strictures in survivors of aluminum phosphide poisoning.

\section{(95) Epidemiology and Types of Ingested Poisons Seen in Emergency and Trauma Department, Kuala Lumpur Hospital}

Suresh Naidu, Mahathar Abdul Wahab, Mohd Idzwan Zakaria, Hidayah Shafie, Datuk Abu Hassan Ansari Abdullah.

Kuala Lumpur Genaral Hospital, University Malaya Medical Centre, Kuala Lumpur, Malaysia

Introduction: Acute poisoning is a common cause of Emergency Department attendance in most countries and most fatal cases happen in low- to middle-income countries. It is important for us to study the type of poisoning to detect changing pattern of poisoning occurring in population. Most of poisoning occurs by oral route. This study focuses on ingested poisoning in urban population of Kuala Lumpur since poisoning studies in this urban population is so far never been done. Methods: A retrospective observational study done by tracing all available patient folders who attended to the Emergency Department due to poisoning from 1 June 2006 to 30 June 2009 for adult cases and 1 January 2007 to 31 December 2009 for paediatric cases. Records were traced from ICD 10 classification by record office. Data from patients who are involved only in ingested poisoning are included in the study and transferred to data collecting form. Analysis done using SPSS and chi square analysis is done when applicable. Result: There is significant correlation between patients' background and poisoning. Three hundred fifty-eight adult patients and 50 paediatric patients satisfy the criteria. Females account for $76 \%$ of poisoning, while among racial profile, Indians result in $44 \%$ of poisoning. Self-harm are most common cause of poisoning in adults, particularly between 15 and 30 years old and incidence of accidental poisoning become more common after 30 years old. Virtually $100 \%$ poisoning above 60 years old is accidental in nature in this study group. Pharmacological poisoning is the most common poisoning accounting for $74 \%$ of total poisoning in adults, with paracetamol poisoning accounting for $55 \%$ cases of pharmaceutical poisoning. In paediatrics group, accidental poisoning is more common and incidence is highest below 5 years old.

Conclusion: The study reveals high incidence of self-harm poisoning particularly among young adult females, and rampant pharmacological poisoning among urban community with paracetamol being most commonly abused drug. As with other studies, ethnic Indians have highest prevalence of poisoning among races.

\section{(96) Matrine Poisoning After Ingestion of Potency- Enhancing Product}

Ho Yin Chan, Wing Lun Chan, Ka Yuen Tang, Fu Ng. Accident and Emergency Department, Caritas Medical Centre, Hong Kong

Introduction: Many 'natural' non-prescription potencyenhancing products are available in Hong Kong with unknown ultimate sources. We describe a case of matrine (苦参碱) poisoning followed by ingestion of sexual enhancement capsules presenting with gastrointestinal and neurological toxicities. Case: In 2009, a 36-year-old man presented to our Emergency Department with generalized weakness, dizziness and repeated vomiting $6 \mathrm{~h}$ after taking four capsules of potencyenhancing agent. He was afebrile with stable vital signs. Clinically he looked tired and was mildly dehydrated. 
Neurological, abdominal and cardiovascular examinations were unremarkable. His spot blood glucose and routine blood results were normal except hypokalaemia (potassium $2.9 \mathrm{mEq} / \mathrm{L}$ ). Electrocardiogram (ECG) showed sinus rhythm and normal QTc interval without any ischaemic change. Patient was admitted for supportive treatment and rehydration. Potassium level was normalized after replacement. His gastrointestinal and neurological symptoms also improved. He was discharged the next day. On follow-up, he recovered completely and his urine specimen detected matrine. During that period, three other patients were reported to develop similar symptoms after taking the same named product. Their urine samples all identified matrine. It was not listed in the insert of the product and the reason of its presence remained unclear. The related health authority was notified about the cluster of cases.

Discussion: Matrine is the main toxic ingredient in $\mathrm{Ku}$-shen 苦参 (sophora falvescentis roots) and Shan-dou-gen山豆根 (sophora tokinensis roots). Its toxicities include protracted vomiting, dizziness, muscular weakness and respiratory paralysis. Coma and residual neurological damage have been reported. It can also induce QTc prolongation in ECG. Treatment is mainly supportive. Poison outbreaks from sexual enhancement products of dubious origin were occasionally reported. Public warnings to avoid these 'health supplements' are necessary because of their potential threat to men worldwide.

\section{(97) An Innovative Multi-model on Managing Suicide an Innovative Multi-model on Managing Suicide}

Tahereh Seghatoleslam ${ }^{1}$, Hussein Habil ${ }^{1}$, Omid Mehrpoor ${ }^{2}$, Esmael Farzaneh ${ }^{3}$.

${ }^{1}$ Malaya University Centre of Addiction Sciences (UMCAS), Kuala Lumpur, Malaysia.

${ }^{2}$ Birjand Medical University, Birjand, Iran.

${ }^{3}$ Ardebil Medical University, Ardebil Iran

Background: Recently in a new perspective, researchers suggested that a multidimensional approach to control suicide can be applied by clinicians in a time-limited setting. Methodology: In this study through an Internet search (MEDLINE \& Cochrane) and a literature review, we suggested an innovative multi-model on managing adult suicide. Results: We suggested an empirically supported treatment approach for suicidality that is specially tailored to today's psychological-managed care environment. The authors present an innovative multi-modal framework of psychological management to help the clinicians rapidly determine and accomplish essential treatment goals, taking into account the severity, chronicity, and diagnostic complexity of the patient's symptoms. Carefully structured, yet flexible enough for the realistic of step by step practice, the multi-model treatment offers guidance on managing all aspects of suicide behavior. It is fully compatible with current goal standards of psychological management and uniquely designed for maximum effectiveness in the clinical time-limited setting.

Suggestion: The application of the innovative multi-model on managing suicide might be used by an expert teamwork in prevention, psycho education, and treatment of adult suicide.

(98) Diazinon Exposure Reduces Trabecular and Cortical Bone Mineral Density

Roya Lari ${ }^{1}$, Marjan Hezb Elahi ${ }^{1}$, Parisa Lari ${ }^{2}$.

${ }^{1}$ Department of Biology, Ferdowsi University of Mashhd, Mashhad, Iran.

${ }^{2}$ Mashhad University of Medical Sciences, Mashhad, Iran

Diazinon (O,O-diethyl-O-[2-isopropyl-6-methyl-4-pyrimidinyl] phosphorothioate) is an organo-phosphate insecticide that has been used worldwide in agriculture and domestic. A variety of environmental deleterious and pathological effects of diazinon has been shown. In this study, we aimed to investigate the effects of diazinon on bone loss. For this purpose, animals were initially divided into three groups. Corn oil used as a control and diazinon 15 and $30 \mathrm{mg} / \mathrm{kg}$ per day in corn oil were given to male wistar rats $(n=6)$ via gavage for 4 weeks. Standardized radiographs were taken from femur bones and the Image J Program was used for measurement of the femoral head, femoral neck, lesser trochanter, greater trochanter, and shaft. This study demonstrated for the first time that diazinon reduces bone mineral density. Mice that were treated with $30 \mathrm{mg} / \mathrm{kg}$ diazinon highly reduced bone density in femoral head, lesser trochanter, greater trochanter, and shaft $(p<0.01)$. Also, in $15 \mathrm{mg} / \mathrm{kg}$ treated mice, bone density of shaft was significantly reduced $(p<0.05)$. These results indicated that diazinon exposure is associated with decrease in trabecular and cortical bone density and might be one of the causes for worldwide increase of osteoporosis.

(99) Effects of Erythropoietin on Electrocardiogram Changes in Carbon Monoxide Poisoning: an Experimental Study in Rats

Amir Hooshang Mohammadpour ${ }^{1,4}$. Mitra Asgharian Rezaee $^{1}$, Seyed Adel Moallem ${ }^{1,2,4}$, Mohsen Imenshahidi ${ }^{1}$, Mahdi Farzadnia ${ }^{3}$.

${ }^{1}$ Department of Pharmacodynamics and Toxicology, School of Pharmacy, Mashhad University of Medical Sciences, Mashhad, Iran.

${ }^{2}$ Medical Toxicology Research Center, Mashhad University of Medical Sciences, Mashhad, Iran.

${ }^{3}$ Department of Pathology, School of Medicine, Emam Reza Hospital, Mashhad University of Medical Sciences, Mashhad, Iran.

${ }^{4}$ Pharmaceutical Research Center, Mashhad University of Medical Sciences, Mashhad, Iran 
Aim: The aim of this study was to define for the first time electrocardiogram (ECG) changes following moderate to severe $\mathrm{CO}$ intoxication in rats, and also to evaluate the effect of erythropoietin (EPO) on observed cardiac disturbances.

Methods: Wistar rats were intoxicated with three different concentrations of CO $(250,1,000$, or 3,000 ppm). EPO was administrated $(5,000 \mathrm{IU} / \mathrm{kg}$, intra-peritoneal injection) at the end of $\mathrm{CO}$ exposure and then the animals were re-oxygenated in ambient air. ECG and heart rate was recorded for evaluating the EPO effects on changes due to $\mathrm{CO}$ intoxication.

Results: ECG changes following CO intoxication included ST segment elevation and depression, T wave inversion, and first-degree AV block. ECG changes reduced significantly in EPO-treated animals.

Conclusion: Our results showed that EPO could inhibit ischemic changes of ECG after $\mathrm{CO}$ poisoning.

(100) Epidemiologic Evaluation of Aluminum Phosphide Mortality in Mazandaran Province 2004-2006

S.K. Ghasempouri ${ }^{1}$, S.M. Naimian ${ }^{1}$, H. Khosrojerdi ${ }^{2}$.

${ }^{1}$ Legal Medicine Research Centre, Legal Medicine Organization, Tehran, Iran.

${ }^{2}$ Medical Toxicology Research Centre, Imam Reza Hospital, Faculty of Medicine, Mashhad University of Medical Sciences, Mashhad, Iran

Rice tablet is a toxin with chemical formula aluminium phosphide (ALP) known as phostoxin and is used extensively in many regions of Iran, especially in Mazandaran Province, because it is an important grain insecticide which has gained popularity among farmers due to its low cost, easy application, and high efficacy. It is used more and more in suicide attempts. Unfortunately, half a tablet can kill people. Because of the rising number of death due to this tablet, in Mazandaran Legal Medicine Center, we did an epidemiologic study to obtain complete information. Data were selected from records and several variables such as age, sex, city, time (month), and etiology were analyzed with SPSS 11.5.

Results: One hundred forty-three deaths $(55.2 \%$ men) were submitted in the databank. More than half (58\%) were young people (age range of 18-30 years old). Most of deaths occurred in spring and summer seasons and it was more common in urban areas. Almost all deaths were due to suicide. Motivation of suicide had not been known in $60 \%$, family quarrel in $16.1 \%$, and other reasons (such as psychiatric diseases, economic problems, addiction, and so on) in $33 \%$.

Conclusion: ALP poisoning and mortality is rising in our community. Urgent collaboration of organizations for changes in production, packing, and distribution of rice tablets is recommended.
(101) Evaluation of Serum Concentrations Following Rectal Administration of Single Dose $(40 \mathrm{mg} / \mathrm{kg})$ Paracetamol in Children

Alizadeh A, Hiradfar M, Afshari R,Balali M, Shojaian R, Sadrosadat Sh.

Medical Toxicology Centre, Imam Reza (p) Hospital, Mashhad University of Medical Sciences Mashhad,Iran

Background: Paracetamol or acetaminophen is an analgesic and antipyretic medicine. These effects are reported at a serum concentration of $4-18 \mu \mathrm{g} / \mathrm{mL}$. Functionally, paracetamol predominantly acts as a central indirect inhibitor of COX-2 enzymes. In some situations such as unconsciousness gastrointestinal intolerance, after surgery where oral feeding is not possible, rectal form of paracetamol is required.

Objective: The objective of this study is to measure the serum concentrations following rectal administration of single-dose $(40 \mathrm{mg} / \mathrm{kg})$ paracetamol in children.

Methods: A single-dose $(40 \mathrm{mg} / \mathrm{kg})$ rectal paracetamol (CILAg LTDSchaffhausen/Switzerland), was administered half an hour after surgery in 20 children (1-8 years) in pediatric surgery ward of Dr. Sheikh Hospital and then serum concentration of paracetamol 4, 8, and $12 \mathrm{~h}$ after administration was measured. Paracetamol level was determined by UV-visible Spectrophotometer (Perkin Elmer USA-550-SE) with precision of $0.1 \mu \mathrm{g} / \mathrm{mL}$.

Results: Mean (SD, min-max) of serum paracetamol levels was $36.4(10.7,10.0-53.0) \mu \mathrm{g} / \mathrm{mL}$ after $4 \mathrm{~h}$ of rectal administration of $40 \mathrm{mg} / \mathrm{kg}$ single dose. Mean values declined to $25.6(10.3,7.0-45.0)$ and $17.6(8.7,17.6,4.0-33.0) 8$ and $12 \mathrm{~h}$ after administration. Mean difference (SD, $95 \% \mathrm{CI}$ ) of serum paracetamol levels from 4 to $8 \mathrm{~h}$ after administration were $10.8(5.8,8.1-13.5 ; P<0.001)$. Similarly, serum paracetamol levels significantly declined from 8 to $12 \mathrm{~h}$ after administration $8.0(3.8,6.2-0.7 ; P<0.001)$.

Conclusion: Serum concentrations of paracetamol, 4 and $8 \mathrm{~h}$ after $40 \mathrm{mg} / \mathrm{kg}$ of rectal form were higher than recommended therapeutic levels and may be a risk for children. In addition, the rate of decreasing is low and may lead to overdosing in repeated doses.

\section{(102) Fatal Poisonings in Rural Southern India-a Retrospective Analysis}

Tanuj Kanchan ${ }^{1}$, Shankar M. Bakkannavar' ${ }^{2}$.

${ }^{1}$ Department of Forensic Medicine and Toxicology, Kasturba Medical College, Mangalore (Affiliated to Manipal University), India.

${ }^{2}$ Department of Forensic Medicine and Toxicology, Kasturba Medical College, Manipal (Affiliated to Manipal University), India 
Objectives: The purpose of the study is to assess the epidemiological variables of fatal poisoning in a rural part of Southern India to understand the magnitude and pattern of poisonings in this region and identify the people at risk.

Materials and Methods: The present hospital-based retrospective analysis was carried out in a tertiary care hospital in a rural part of Southern India. All the poisoning cases autopsied during January 2007 and December 2010 was included in the study. A detailed profile was made based on the hospital and autopsy records, information furnished by the police and reports from the regional forensic science laboratories. The data was statistically analysed using SPSS version 11.0. Chi-square $\left(\chi^{2}\right)$ test was performed to test the significance of each group.

Results: A total of 162 cases of fatal poisonings were recorded during the study period. Males were predominantly affected (66.0 \%), male-female ratio being 1.9: 1. Majority of the victims were Hindus (89.5\%) and aged between 20 29 years $(35.2 \%)$. Mean age was $37.07 \pm 18.3$ years. Agrochemical agents were the main culprit with organophosphates (OPCs) alone were implicated in more than half of the cases, followed by carbamates and rodenticides. The manner of death was suicidal in $86.4 \%$ and accidental in $10.5 \%$ cases. Most of the victims consumed poison in the morning hours between 6 am and 12 noon $(n=62)$ and maximum fatalities were reported in the months of May $(n=19)$. Period of survival ranged from 0 to 26 days. Fatalities $(47.5 \%)$ were reported within 3 days of consumption of poison.

Conclusion: Acute poisonings are a cause of concern in the region. Strict implementation and enforcement of laws, greater control in the sale, and use of agrochemicals are recommended along with better health care facilities in rural India, to prevent poisoning-related mortalities.

\section{(103) Malaysian Hospital Calls for Children Exposure} to Household Cleaning Products: an Analysis of 20062009 National Poison Centre Data

Halilol Rahman M.K. ${ }^{1}$, Rahmat Awang ${ }^{1}$, Sa'ed H. Zyoud ${ }^{1,2}$, Adilah MA ${ }^{1}$, Nur Afni A. ${ }^{1}$.

${ }^{1}$ WHO Collaborating Centre for Drug Information, National Poison Centre, Universiti Sains Malaysia (USM), Penang, Malaysia.

${ }^{2}$ Poison Control and Drug Information Center (PCDIC) and College of Pharmacy, An-Najah National University, Nablus, Palestine

Objective: Household cleaning products (HCP) have been responsible for many accidental poisonings among children. In Malaysia, there is no available published epidemiological data for poisoning among children regarding these products. Thus, the aim of this study is to analyse all referral cases and report all the telephone calls to the National Poison Center (NPC) by hospitals in Malaysia regarding HCP exposure.

Methods: We analysed all calls related to HCP reported to NPC for the period between January 2006 and December 2009. Type of HCP, age, gender, date, route and reason of exposure were evaluated using SPSS version 15.0.

Results: There were 1,106 telephone HCP-related enquiries which constitute approximately $49.5 \%$ of all household poisoning cases collected in the same period. HCP exposures accounted for 14.8 and $34.6 \%$ of all poisoning cases in children and adult, respectively. Majority of the cases in children were reported to be accidental $(92.2 \%)$. Bleach (i.e. sodium hypochlorite) was the most commonly involved product $(n=168)$ followed by detergent $(n=70)$ and antiseptic products (i.e. chloroxylenol; $n=27$ ). Exposure to HCP occurred mainly via ingestion ( $98.8 \%$ ) followed by inhalation $(0.6 \%)$. The number of poisoning calls related to HCP was observed to be increased annually from 38 calls $(11.4 \%)$ in 2006 to 134 calls $(40.4 \%)$ in $2009(P<0.001)$. Information on children outcome was not available.

Conclusion and Recommendations: There has been a significant increase in the number of calls received by the NPC involving children who have accidentally taken cleaning products. Parents and childcare providers must ensure that HCP should be kept in a locked cabinet and out of children's sight and reach. Improved methods of follow-up are needed if adequate information is to be made available to support management and provide advice to the healthcare professionals. Finally, another national study needs to be carried out to better estimate the pattern of the HCP use and pattern of inappropriate products storage.

\section{(104) The Effects of Health Educational Intervention on Blood Lead Levels in Workers in a Lead-Contaminated Factory}

Mohammadian Bajgiran AH, Afshari R.

Medial Toxicology Centre, Mashhad University of Medical Sciences, Mashhad, Iran

Introduction: The most common exposure to lead among adults is occupational in the whole world, and it also is a health problem in Iran. We conducted this study to determine the prevalence of clinical findings and the effects of health educational intervention on blood lead levels in leadcontaminated factory in northeastern Iran.

Methods: We visited workers in a lead-contaminated factory and collected data by direct history taking and physical examination. After the first visit, we installed ventilation in the factory, taught them some health educational information, and for high blood lead levels, we did conventional medical intervention. Blood and urine lead concentrations were measured before and after of interventions. 
Results: In total, 87 skilled workers in lead-contaminated factory were studied. The main objective clinical findings were the presence of hypersensitivity $(10 \%)$, weight loss ( $2 \%)$, fatigue and weakness $(9 \%)$, respiratory problems (50\%, includes $\operatorname{cough}(9 \%)$, dyspnea $(10 \%)$, hoarseness (7\%), and asthma (4\%)), dyspepsia (5\%), constipation (4\%), xerostomia (4\%), abdominal pain (5\%), nausea (6\%), dysuria (5\%), edema (3\%), aggressiveness (42\%), headache (12\%), knee pain (17\%), and general pain (6\%). Blood lead level mean (SD, min-max) was $122(61,16-$ 483) $\mu \mathrm{g} / \mathrm{L}$. Urine lead level was $23(12,1-35) \mu \mathrm{g} / \mathrm{L}$. Blood lead levels were statistically correlated with time elapse from our intervals ( $p=0.044, r=-0.316$ ).

Conclusion: Lead poisoning is preventable. Lead affects multiple body systems and can cause permanent damage, which can be prevented with health care and medical attention for every worker in lead-contaminated factory.

(105) Adjusted Study of Clinical and Lab Findings of Deceased Patients in Imam Reza Hospital Toxicology Ward with Mashhad Forensic Medicine Autopsy Findings from Shahrivar 1388 to Shahrivar 1389 (2009-2010)

Seyed Khosro Ghasempuri ${ }^{1}$, Reza Afshari ${ }^{1}$, Hamid Reza Rahimi $^{2}$, Golamreza Gonabadi ${ }^{3}$, Hamid Mohabati ${ }^{3}$.

${ }^{1}$ Medical Toxicology Research Centre, Imam Reza Hospital, Faculty of Medicine, Mashhad University of Medical Sciences, Mashhad, Iran.

${ }^{2}$ Mashhad University of Medical Sciences, Mashhad, Iran.

${ }^{3}$ Islamic Azad University, Mashhad Iran

Introduction: Human poisoning presents in diverse forms and can either have an indolent or a fulminate course. Sometimes, it is hard to come up with a firm diagnosis for the cases with prompt chronologies. One of the advantages of Imam Reza Toxicology Ward is being close to the Autopsy Theatre, Forensic Pathology, and Forensic Toxicology Laboratory. This paper is the Adjusted Study of Clinical and Lab Findings of Deceased Patients in Imam Reza Hospital Toxicology Ward with Mashhad Forensic Medicine Autopsy Findings from Shahrivar 1388 to Shahrivar 1389.

Methods: This is a retrospective study, investigating the clinical, lab, and forensic pathology findings of expired patients who were suspected to have experienced acute poisoning in the period of 1 year.

Results: Documents of 116 deceased patients with the primary diagnosis of acute poisoning were extracted from the archive. Only 60 of these cases were autopsied, from which, $58.3 \%$ were male, $60 \%$ had been married, $18.3 \%$ had the history of smoking, $25 \%$ had the history of opiates consumption, $7.1 \%$ had been addicted to drugs, and $6.8 \%$ had tattooing. ECG findings of 22 patients $(36.7 \%)$ demonstrated sinus tachycardia, which proved to be the most common. In primary physical examination, 17 subjects had been unconscious, 28 subjects were diagnosed correctly by poisoning, 26 subjects had committed suicide, and 19 patients were hospitalized without any diagnosis. According to ABG findings, 17 patients had had metabolic acidosis and 11 had had respiratory acidosis. Ten subjects had the history of either former poisoning or suicidal attempts. The major causes of death were drug abuse and its complications ( $25 \%)$, organophosphorus compounds (10\%), and rice pills (5\%). Clinical findings and autopsy accorded completely for 28 subjects. For 22 patients, clinical findings and autopsy showed partial accordance, and for the other 10, the clinical and autopsy findings did not match.

Conclusion: Suicidal attempts with rice pills and poisoning with organophosphorus compounds and opiates are the most common causes of death due to acute poisoning because of their potentially fulminate course. On the other hand, we have to admit that $17 \%$ of the triages to the toxicology ward were incorrect and it is need to be more careful in referring unconscious patients.

\section{(106) Sensitizing and Engaging Youth on the Dangers of Drug Abuse Using Visualisation in Participatory Program (VIPP): the Malaysian Experience}

M. Nazri MT, Sulastri S, Haslina H, Maizurah O, Saed Z. National Poison Centre (NPC), Universiti Sains Malaysia

Introduction: In 2002, it was estimated that approximately 59,243 to 77,196 youths in Malaysia were categorized as substance abusers. The National Anti-Drug Agency recorded the involvement of youth as substance abusers as early as 13 years of age. Realizing the need to educate youths on the dangers of these substances, the National Poison Centre (NPC) conducted a number of Anti-Drug Education and Prevention Programs (PPDa) in schools using a new technique called Visualisation in Participatory Program (VIPP). This technique is aimed to enhance learning and effectively sensitize and engage youths to understand the dangers of substances of abuse (SOA).

Objective: The objective of this paper is to share the VIPP experience and how it has enhanced learning through greater participation, interaction, and fun.

Methods: On a yearly basis, the NPC is invited to provide about 30 PPDa to primary and secondary schools in the Penang and Kedah states. NPC designs its own PPDa module incorporating VIPP facilitation tools. Training-ofTrainers workshops are conducted for students in schools using the VIPP module. "World café" is among the tools popularly used to encourage student's participation through rotating discussion. Other tools include "buzz group" to induce quick and organized discussion, "lifeboat" to 
energize and divide student into groups, and many others. At the conclusion of the workshops, quick evaluation on the percentage of SOA understanding and ability to practice of the gained knowledge in daily life is done.

Result: Almost half the students involved were able to solve the tasks given during the workshops and could answers SOA-related questions. These students who call themselves "Don't Break My Heart" then voluntarily conduct peerbased interactive programs like exhibits, workshops, signature campaign, and social network portal at their school to encourage interest among their peers to learn about SOA in a more interactive and effective manner.

Conclusion and Recommendation: The VIPP method allows youths to better understand the topics introduced and creates opportunities to generate new ideas through stimulation of participatory learning. Further studies are needed to assess the knowledge of youths and to evaluate the effectiveness of VIPP as a role to enhance learning

(107) Sustaining 24-h Poisoning Call Service: the Malaysian Experience

Haslina H., Adilah M.A., Sazaroni M.R., Rahmat A., Sa'ed H.Z.

National Poison Centre of Malaysia, Universiti Sains Malaysia, 11800 Minden, Penang, Malaysia

Introduction: Poisoning cases worldwide have increased through the years. Poison centres around the world, both in developed and developing countries, are obliged to offer a 24-h poisoning call service. Many centres, especially in the developing countries, however, have not been able to do this due to limited personnel and budgetary constraint.

Objective: The objective of this study is to share the experience of the Malaysian National Poison Center (MNPC) in handling after-office-hour calls from home.

Methods: All poisoning cases documented by the centre for the period between 2006 and 2010 were reviewed and analysed. Calls received on work days, between 8 am to $5 \mathrm{pm}$, are serviced from the centre. Beyond office hours, on weekends and public holidays, calls are serviced from home and are referred to as after-office-hour calls. On regular work days, after-office-hour calls are handled by a duty officer on-call. For weekends and public holidays, the duty is shared by two officers: one on day shift, and the second from evening until the following morning. The officer-oncall is provided with a mobile phone, computerized databases and a broadband for convenient access to internet service.

Results: During the four-year period, the total number of poisoning calls received by MNPC was 14,534 . Of this total, $37.8 \%$ were received during office hours while the remaining $62.2 \%$ were received after the office closed. The after-office calls were found to increase annually from $33.1 \%$ to $54.7 \%$. Majority of the calls were handled successfully by the duty officer and reports of assistance required after office hours were minimal.

Conclusion: The significant increase in the number of afteroffice-hour calls during the 4-year period is a clear indication that the 24-h poisoning call service is very much required. For centres with limited personnel and funding, the after-office-hour service can be operated from home, provided that the duty officer is equipped with adequate references and communication device. However, certain limitations such as the absence of a mobile telephone system for recording poisoning call conversations and the requirement for a staff to work not more than $48 \mathrm{~h}$ a week will need to be addressed to ensure that the quality of service provided is not compromised.

\section{(108) The Role of Poison Centers in Chemical Safety and Management: the Philippine Experience}

Panganiban, LR.

National Poison Management and Control Center, UP College of Medicine-Philippine General Hospital, Manila, Philippines

While chemicals are acknowledged as important inputs to increase productivity in industrial and agricultural settings, their indiscriminate use may result to poisoning of individuals and populations. With the increasing use of chemicals in the country comes the challenge in ensuring safety and keeping people from harm's way. With this background, the role of poison centers has expanded from being centers for treatment of poisoning cases, poison information, and analytical toxicology and training/education to various other roles that have national and international relevance as well. The Philippine national poison control center had to step out of the hospital's walls and assumed other roles in chemical safety and management. These roles included responding to chemical incidents in communities, raising toxicologic issues of national concerns, and rallying towards poisoning prevention. These functions have made the poison center's existence relevant to the times. This presentation will provide an overview of the growth of the national poison center and other centers in the country, description of activities undertaken in fulfilling its new roles, and lessons learned in the process. Example of activities included the conduct of health surveillance activities, drafting of recommendations on national policies on poisoning-related matters and issuance of health advisories. One of its important lessons is the essence of partnership and networking, in that, strength comes in numbers. "A cord of three strands is not quickly torn apart." 
(109) Acute Toxicity and Biochemistry Studies of Combined Aqueous Extracts of Curcuma longa and Quercus infectoria

Nahlah Elkudssiah Ismail ${ }^{1,2}$, Tommy Joulianto Bustami Effendi $^{3}$, Aida Azlina $\mathrm{Ali}^{4}$, Mizaton Hazizul Hasan ${ }^{4}$. ${ }^{1}$ Clinical Pharmaceutics Research Group (CPRG), Faculty of Pharmacy, Universiti Teknologi MARA (UiTM) Puncak Alam Campus, 42300 Bandar Puncak Alam, Selangor, Malaysia.

${ }^{2}$ Inhalational Delivery Research Unit (IDRU), Faculty of Pharmacy, Universiti Teknologi MARA (UiTM) Puncak Alam Campus, 42300 Bandar Puncak Alam, Selangor, Malaysia.

${ }^{3}$ Nutraceutical, Cosmeceutical and Complementary Medicine Research Unit, Faculty of Pharmacy, Universiti Teknologi MARA (UiTM) Puncak Alam Campus, 42300 Bandar Puncak Alam, Selangor, Malaysia.

${ }^{4}$ Pharmaco-toxicology Research Group, Faculty of Pharmacy, Universiti Teknologi MARA (UiTM) Puncak Alam Campus, 42300 Bandar Puncak Alam, Selangor, Malaysia

Curcuma longa L. (Zingiberaceae) (well-known as turmeric (English) and kunyit or haldi among Malaysians) and Quercus infectoria (known as manjakani (Malay) and majuphal (Indian) among Malaysians) are widely used in herbal preparation for post-natal care to promote wellness after childbirth especially in wound healing and weight management. The aqueous extract of $Q$. infectoria galls and C. longa rhizomes was demonstrated to have high hydrolysable tannin and curcumin, respectively. However, not many studies were done to look into the safety of these plants especially when used in combination. This study was carried out to determine the acute toxicity and biochemistry profiles of these combined aqueous plant extracts. According to OECD guideline, male and female $(n=6$ for each sex in three groups) Mus muskulus mice were given 2 and $5 \mathrm{~g} / \mathrm{kg}$ of the combined aqueous extract with ratio of $C$. longa/ $Q$. infectoria 2:1 orally and control were given normal saline. The body weight, and food and water intake were recorded. The animals were observed for 14 days after the administration of the respective extract. After 14 days, the animals were sacrificed and the blood samples were collected for biochemistry analysis that include ALT, AST, albumin, calcium, cholesterol, glucose, total protein, triglycerides, and urea. No death of mice was reported from day 1 until day 14. There were slight increased in body weights however not significant. Results demonstrated that combination of $Q$. infectoria nut galls and $C$. longa rhizomes aqueous extract was not toxic. There were reduced level of albumin, cholesterol, glucose, total protein, and urea. However mixed biochemistry profiles between studied males and females in fed and control group were significantly due to gender differences. In conclusion, combination of $Q$. infectoria and $C$. longa aqueous extract was considered as safe and may be potential adjunctive anti-obesity agent marketable for weight management.

\section{(110) Chemical Burns Due to Accidental Exposure of Sodom Apple—a Case Report}

B Suresh Kumar Shetty, Jagadish Rao P P, Srinidhi, Amith. ${ }^{I}$ Department of Forensic Medicine Kasturba Medical College, Mangalore Manipal University, Manipal, India.

${ }^{2} A J$ Institute of Research and Medical Science Mangalore, Department of Forensic Medicine Kasturba Medical College, Mangalore Manipal University, Manipal, India

A chemical burn is a special type of burn injury, caused by a chemical agent. It occurs when living tissue is exposed to such a corrosive, irritant chemical compound, most commonly a base or an acid. These burns do not need a source of heat and can be extremely painful, as they evolve very quickly. Calotropis procera produces copious amounts of latex, which has been shown to possess several pharmacological properties. Its local application produces intense inflammatory response and produce chemical burns. Accidental exposure to the latex has been reported to cause inflammation of the skin and eyes. Ocular injury from this plant can be mechanical, or more commonly, toxic, due to the exposure to its latex. We report a case with a spectrum of skin toxicity following accidental exposure to the latex of C. procera (Sodom apple).

(111) Determination of Caprolactam and 6Aminocaproic Acid in Human Urine Using Hydrophilic Interaction Liquid Chromatography Tandem Mass Spectrometry

Ya-Hsueh $\mathrm{Wu}^{1}$, Ming-Ling Wu ${ }^{1}$, Wei-Lan $\mathrm{Chu}^{1}$, ChenChang Yang ${ }^{2}$, Jou-Fang Deng ${ }^{2}$.

${ }^{1}$ Department of Medicine, Taipei Veterans General Hospital, Taipei, Taiwan.

${ }^{2}$ National Poison Centre, Taipei Veteran General Hospital, Taipei, Taiwan.

A simple and rapid assay based on hydrophilic interaction liquid chromatography (HILIC) with tandem mass spectrometry has been first developed and validated for simultaneous determination of caprolactam (CA) and 6-aminocaproic acid (6-ANCA) in human urine using 8-aminocaprylic acid as internal standard. A $20 \mu \mathrm{L}$ aliquot of urine was injected directly into the liquid chromatography tandem mass spectrometry (LC-MS/MS) system. The analytes were separated on a Phenomenex Luna HILIC column with gradient elution. Detection was performed on Triple Quadrupole LC-MS in positive ions Multiple Reaction Monitoring mode using electrospray ionization. The calibration curves were linear $\left(r^{2} \geq\right.$ 
$0.995)$ over the concentration range from 62.5 to $1,250 \mathrm{ng} / \mathrm{mL}$ for $\mathrm{CA}$ and 31.25 to $1,000 \mathrm{ng} / \mathrm{mL}$ for 6-ANCA. The detection limits of CA and 6-ANCA were 62.5 and $15.6 \mathrm{ng} / \mathrm{mL}$, respectively (signal-to-noise ratio $=3$, with $20 \mu \mathrm{L}$ injected). The intra-day and inter-day precisions were within 6.3 and $8.9 \%$, respectively. The intra-day and inter-day accuracies were between -5.8 and $12 \%$, and between -6.3 and $9.4 \%$, respectively. The method proved to be simple and time efficient, and was successfully applied to evaluate the kinetics of caprolactam in one unusual case of caprolactam poisoning.

\section{(112) The Effect of the Lead Acetate (Pb) on Tracheal} Responsiveness of Sensitized Guinea Pigs

Mohammad Hossein Boskabady ${ }^{1}$, Sedighe Jalali ${ }^{1,2}$, Tahere Farkhondeh $^{1,3}$, Goltaj Bayrami ${ }^{1}$.

${ }^{1}$ Department of Physiology and Pharmaceutical Research Centre, Mashhad University of Medical Sciences, Mashhad, Iran.

${ }^{2}$ Department of Biology, Islamic Azad University, Sciences and Research Unit, Tehran, Iran

Objective: The objective of this study is to examine the effect of lead acetate on tracheal responsiveness of nonsensitized and sensitized guinea pigs.

Methods: Guinea pigs were randomly allocated into control (group $\mathrm{C}$ ), sensitized (group $\mathrm{S}$ ), and four $\mathrm{Pb}$-exposed groups: $\mathrm{Pb}(0.1 \mathrm{M})$-exposed (group $\mathrm{Pb} 0.1), \mathrm{Pb}(0.2 \mathrm{M})$ exposed (group $\mathrm{Pb} 0.2), \mathrm{Pb}(0.1 \mathrm{M}$ )-exposed during sensitized (group $\mathrm{Pb} 0.1$ during $\mathrm{S} 0.1$ ), and $\mathrm{Pb}(0.2 \mathrm{M}$ )exposed after sensitization (group $\mathrm{Pb} 0.1$ post-S), $\mathrm{Pb}$
$(0.2 \mathrm{M}$ )-exposed after sensitized (group $\mathrm{Pb} 0.2$ post-S), $(n=7$, for all groups). Guinea pigs were sensitized with i.p. injected and inhaled ovalbumin (OA). Control animals received saline instead of $\mathrm{OA}$. $\mathrm{Pb}$-exposed groups were exposed to lead acetate 0.1 and $0.2 \mathrm{M}$ for $1 \mathrm{~h}$, three times weekly for 15 days. Tracheal responses (TR) to methacholine were measured by performing concentration response curve of tracheal smooth muscle to methacholine and determining its effective concentration causing $50 \%$ of maximum response $\left(\mathrm{EC}_{50}\right)$. Tracheal responses to $0.1 \% \mathrm{OA}$, relative to contraction obtained by $10 \mu \mathrm{M}$ methacholine were also examined.

Results: The TR to both methacholine and OA of $\mathrm{S}$ guinea pigs were significantly higher than those of controls $(p<0.05$ and $p<0.001$ for $\mathrm{EC}_{50}$ and $\mathrm{OA}$, respectively). In non-sensitized animals exposed to $\mathrm{Pb}$ TR to methacholine was not statistically different but their TR to OA was significantly lower compared to controls $(p<0.05$ for both $\mathrm{Pb} 0.1$ and $\mathrm{Pb} 0.2)$. TR to both methacholine and $\mathrm{OA}$ in these groups was significantly lower than that of group $\mathrm{S}(p<0.01$ for all cases). In animals exposed to $\mathrm{Pb}$ after sensitization, the TR to both methacholine and $\mathrm{OA}$ were the same as group $\mathrm{S}$ and significantly lower than group C. TR to methacholine in these groups was significantly higher than that of group $\mathrm{S}(p<0.05$ for methacholine and $p<0.001$ for $\mathrm{OA}$ in all cases). In addition, in animals expose to $\mathrm{Pb}$ after sensitization, the TR to OA was significantly higher than group $\mathrm{S}(p<0.001$ for both $\mathrm{Pb} 0.1$ and $\mathrm{Pb} 0.2)$.

Conclusions: These results showed that lead acetate can cause tracheal hyperresponsiveness to OA in guinea pigs. In addition, the results showed that TR to OA further enhanced in animal exposed to $\mathrm{Pb}$ after sensitization. 